Periodic Solutions near Equilibria of Symmetric Hamiltonian Systems

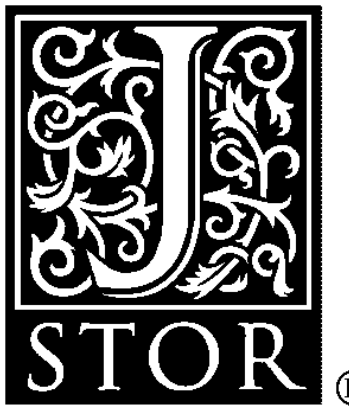
Author(s): J. A. Montaldi, R. M. Roberts, I. N. Stewart

Source: Philosophical Transactions of the Royal Society of London. Series A, Mathematical and Physical Sciences, Vol. 325, No. 1584, (May 25, 1988), pp. 237-293

Published by: The Royal Society

Stable URL: http://www.jstor.org/stable/38039

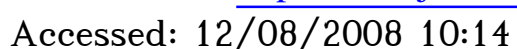

Your use of the JSTOR archive indicates your acceptance of JSTOR's Terms and Conditions of Use, available at http://www.jstor.org/page/info/about/policies/terms.jsp. JSTOR's Terms and Conditions of Use provides, in part, that unless you have obtained prior permission, you may not download an entire issue of a journal or multiple copies of articles, and you may use content in the JSTOR archive only for your personal, non-commercial use.

Please contact the publisher regarding any further use of this work. Publisher contact information may be obtained at http://www.jstor.org/action/showPublisher?publisherCode=rsl.

Each copy of any part of a JSTOR transmission must contain the same copyright notice that appears on the screen or printed page of such transmission.

JSTOR is a not-for-profit organization founded in 1995 to build trusted digital archives for scholarship. We work with the scholarly community to preserve their work and the materials they rely upon, and to build a common research platform that promotes the discovery and use of these resources. For more information about JSTOR, please contact support@ jstor.org. 


\title{
PERIODIG SOLUTIONS NEAR EQUILIBRIA OF SYMMETRIC HAMILTONIAN SYSTEMS
}

\author{
By J. A. MONTALDI, R. M. ROBERTS AND I. N. STEWART \\ Mathematics Institute, University of Warwick, Coventry CV4 7AL, U.K.
}

(Communicated by E. C. Zeeman, F.R.S. - Received 17 March 1987)

\section{GONTENTS}

0. Introduction

(a) Existence

(b) Stability

1. Existence OF PERIODic SOlutions

2. EQUIVARIANT SYMPLECTIC LINEAR MAPPINGS $\quad 248$

(a) Statement of results $\quad 248$

(b) Isotypic decomposition of a symplectic representation $\quad 250$

(c) Decomposition of $\operatorname{Sp}_{G}(\mathrm{~V}) \quad 253$

(d) Eigenvalues of equivariant symplectic mappings $\quad 254$

3. Stability OF EQUilibrium POINTS

4. Degomposition of Floquet operators $\quad 256$

(a) Decomposition of the tangent space $\quad 257$

(b) Decomposition of Floquet operators $\quad 260$

(c) Relation between the actions of $\Gamma$ and $\Gamma \times \mathbb{R} \quad 262$

5. Stability of Periodic solutions

(a) Perturbation of the Floquet operator $\quad 265$

$\begin{array}{ll}\text { (b) Krein theory } & 267\end{array}$

(c) Eigenvalues far from $1 \quad 268$

6. Deforming the action of $\bar{\Sigma} \quad 269$

(a) $\Sigma$-equivariance and eigenvalues near $1 \quad 269$

(b) Proof of the stability theorem $\quad 273$

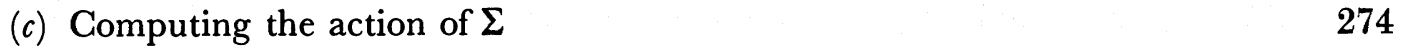

(d) Calculation of $\Gamma_{\mu} \quad 275$

7. Simple EXAMPLes $\quad 277$

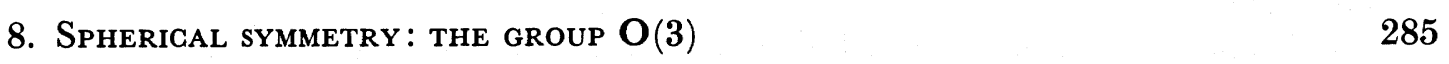

(a) Existence of periodic trajectories $\quad 285$

(b) Eigenvalues forced to $1 \quad 288$

(c) The residual Floquet operator $\quad 289$

REFERENGES $\quad 292$ 
We consider the effects of symmetry on the dynamics of a nonlinear hamiltonian system invariant under the action of a compact Lie group $\Gamma$, in the vicinity of an isolated equilibrium: in particular, the local existence and stability of periodic trajectories. The main existence result, an equivariant version of the Weinstein-Moser theorem, asserts the existence of periodic trajectories with certain prescribed symmetries $\Sigma \subset \Gamma \times \mathbf{S}^{1}$, independently of the precise nonlinearities. We then describe the constraints put on the Floquet operators of these periodic trajectories by the action of $\Gamma$. This description has three ingredients: an analysis of the linear symplectic maps that commute with a symplectic representation, a study of the momentum mapping and its relation to Floquet multipliers, and Krein Theory. We find that for some $\Sigma$, which we call cyclospectral, all eigenvalues of the Floquet operator are forced by the group action to lie on the unit circle; that is, the periodic trajectory is spectrally stable. Similar results for equilibria are described briefly. The results are applied to a number of simple examples such as $\Gamma=\mathbf{S O}(2), \mathbf{O}(2), Z_{n}, D_{n}, \mathbf{S U}(2)$; and also to the irreducible symplectic actions of $\mathbf{O}(3)$ on spaces of complex spherical harmonics, modelling oscillations of a liquid drop.

\section{INTRODUCTION}

In this paper we use representation-theoretic methods to prove some general existence and stability results for periodic trajectories near equilibrium points of nonlinear hamiltonian systems that are invariant under the action of a compact Lie group of symmetries.

\section{(a) Existence}

First suppose that no symmetries are present. Let $\mathscr{H}$ be a hamiltonian function on a $2 n$ dimensional phase space (symplectic manifold) $\mathrm{P}$, and let $p$ be a non-degenerate minimum of $\mathscr{H}$, that is, $\mathrm{D} \mathscr{H}_{p}=0$ and $\mathrm{D}^{2} \mathscr{H}_{p}$ is positive definite. The eigenvalues of the linearization $\mathrm{L}$ of the vector field at $p$ are purely imaginary pairs $\left\{ \pm \lambda_{1}, \ldots, \pm \lambda_{n}\right\}$. Liapunov (1907) proved the centre theorem: if some $\lambda_{i}$ is non-resonant then there exists a smooth two-dimensional submanifold of $\mathrm{P}$, passing through $p$, and intersecting each energy level near $p$ in a periodic trajectory with period near $2 \pi /\left|\lambda_{i}\right|$, see Abraham \& Marsden (1978). By 'non-resonant' we mean that $\lambda_{j}$ is not an integer multiple of $\lambda_{i}$ for any $j \neq i$. In a celebrated generalization, Weinstein (I973) and Moser (1976) proved that even when there is resonance, there still exist $n$ periodic trajectories on each energy level near $p$, each having period near $2 \pi /\left|\lambda_{i}\right|$ for some $i$. In general, these no longer form smooth families through $p$ (see, for example, Duistermaat I984). The theorems of Liapunov, Weinstein, and Moser apply to equilibria other than minima of $\mathscr{H}$, and so do the results of this paper, but for the purposes of this introduction it is simpler to describe the results in the above setting. The importance of these results lies in the fact that periodic trajectories may often be used as a framework around which the dynamics of a system near an equilibrium can be organized. For example the K.A.M. theorem ensures that many periodic orbits are surrounded by invariant $n$-dimensional tori, hence lie at the centre of regions of stability, neglecting the exponentially small effect of Arnold diffusion. See Arnold (1978) and Hénon ( 1983 ).

Suppose now that $\Gamma$ is a compact Lie group (not necessarily connected, so that we include finite groups) acting symplectically on $\mathbf{P}$, that is, by 'canonical transformations'. Let $\mathscr{H}$ be invariant under the action of $\Gamma$ and let $p$ be a fixed point for $\Gamma$. In general such a group action will force some of the $\lambda_{i}$ to be equal, restricting the applicability of the Liapunov centre theorem. 
The Weinstein-Moser theorem can still be used, but the estimate of the number of periodic trajectories is usually weak. For instance, in the system of Hénon \& Heiles (1964) at low energy, the Weinstein-Moser theorem predicts two families of periodic trajectories, whereas in fact there are eight. For the spherical pendulum the Weinstein-Moser theorem also predicts two solutions, but there are infinitely many, falling into two orbits under the symmetry group $\mathbf{O}(2)$. Our results predict all of these trajectories, as shown in $\S 1$.

The reason for this is that the standard Weinstein-Moser theorem does not (directly) give information about the symmetry groups of periodic trajectories. If $u(t)$ is a periodic trajectory with period $T$, so that $u(T)=u(0)$, then so is $\gamma u(t)$ for all $\gamma \in \Gamma$. Phase-shifted solutions $u(t+\theta)$ are also periodic trajectories. In fact there is a natural action of $\Gamma \times \mathbf{S}^{1}$ defined by

$$
(\gamma, \theta) u(t)=\gamma u(t+T \theta / 2 \pi)
$$

preserving periodic trajectories. Here we identify the circle group $\mathbf{S}^{1}$ with $\mathbb{R} / 2 \pi \mathbb{Z}$. The symmetry group of $u(t)$ is the group

$$
\Sigma_{u}=\left\{(\gamma, \theta) \in \Gamma \times \mathbf{S}^{1} \mid \gamma u(t+T \theta / 2 \pi)=u(t)\right\}
$$

The set

$$
\mathcal{O}_{u}=\{\gamma u(t) \mid \gamma \in \Gamma, t \in \mathbb{R}\}
$$

is diffeomorphic to the quotient $\left(\Gamma \times \mathbf{S}^{\mathbf{1}}\right) / \Sigma_{u}$. The projection $\mathrm{H}$ of $\Sigma_{u}$ into $\Gamma$ consists of all elements of $\Gamma$ that take $u(t)$ into a phase-shifted version of itself. However, it is important to work with $\Sigma_{u}$ rather than $\mathrm{H}$ because distinct $\Sigma_{u}$ may have the same projection $\mathrm{H}$. For instance this occurs in the Hénon-Heiles system: see example $1.3 b$.

For simplicity of exposition in this introduction let us suppose that $\lambda_{1}=\ldots=\lambda_{n}=\lambda$. (Our actual results apply more generally, see $\S 1$.) Then the trajectories of the linearized vector field at $p$, defined on the tangent space $T_{p} \mathrm{P}$, are all periodic with period $2 \pi /|\lambda|$. This flow therefore defines an $\mathbf{S}^{1}$-action which commutes with the linearization of the $\Gamma$-action, the two together yielding an action of $\Gamma \times \mathbf{S}^{1}$ on $T_{p}$ P. Let $\Sigma$ be a subgroup of $\Gamma \times \mathbf{S}^{1}$ and define the fixed-point subspace

$$
\operatorname{Fix}(\Sigma)=\left\{v \in T_{p} \mathrm{P} \mid \sigma v=v \forall \sigma \in \Sigma\right\} .
$$

Our existence result, theorem 1.1, states that there exist at least $\frac{1}{2} \operatorname{dim} \operatorname{Fix}(\Sigma)$ periodic trajectories of the nonlinear system, with periods near $2 \pi /|\lambda|$ and symmetry groups containing $\Sigma$, on each energy level sufficiently close to $p$. The Weinstein-Moser result may be recovered by taking $\Sigma=1$, the trivial subgroup. We also have a version of the Liapunov centre theorem: if $\operatorname{dim} \operatorname{Fix}(\Sigma)=\mathbf{2}$ (and $\Sigma$ is chosen to be maximal with respect to having this fixed-point subspace) then there exists a family of periodic trajectories, with symmetry groups equal to $\Sigma$, forming a smooth two-dimensional submanifold through $p$. Examples suggest that in 'generic' systems with symmetry, our result gives sharp estimates for the number of periodic trajectories with periods near those of the linearized system.

\section{(b) Stability}

After $\S 1$ the rest of the paper is directed at the stability of these periodic trajectories. First recall some definitions. Let $\sigma: \mathrm{P} \times \mathbb{R} \rightarrow \mathrm{P}$ denote the flow generated by $\mathscr{H}$ and for fixed $t \in \mathbb{R}$ write $\sigma_{t}=\sigma(\cdot, t)$. If $u(t)$ is a $T$-periodic trajectory then its Floquet operator is

$$
\left(\mathrm{D} \sigma_{T}\right)_{u(0)}: T_{u(0)} \mathrm{P} \rightarrow T_{u(0)} \mathrm{P} .
$$


Definition 0.1

(a) A $T$-periodic trajectory $u(t)$ is Liapunov stable (with respect to a metric $\|\cdot\|$ on $\mathrm{P}$ ) if for all $\epsilon>0$ there exists $\delta>0$ such that if $v(t)$ is a trajectory satisfying $\|u(0)-v(0)\|<\delta$, then $\inf _{s}\|u(s)-v(t)\|<\epsilon$ for all $t>0$.

(b) A $T$-periodic trajectory $u(t)$ is linearly stable if it is Liapunov stable as a trajectory of the linearization of the vector field about $u(t)$.

(c) A $T$-periodic trajectory $u(t)$ is spectrally stable if the eigenvalues of $\left(\mathrm{D} \sigma_{T}\right)_{u(0)}$ all lie on the unit circle in $\mathbb{C}$.

Linear stability is neither necessary nor sufficient for Liapunov stability, but spectral stability is a consequence of either. For the analogous statements at equilibria see Holm et al. (1985).

In the presence of a group action these definitions of Liapunov and linear stability are not appropriate. For example, consider those periodic trajectories of a spherical pendulum that lie in vertical planes (see example $1.3 a$ ). These are neither Liapunov nor linearly stable: small perturbations can give quasiperiodic trajectories that drift away from the original periodic trajectory. Both types of stability can be recovered only by modifying the definitions, insisting only that the perturbed trajectory remains close to the entire $\Gamma$-orbit of the original periodic trajectory. However, these matters will be dealt with elsewhere: in this paper we concentrate on the weaker notion of spectral stability, whose definition does remain appropriate in the symmetric context. The planar periodic trajectories of the spherical pendulum are spectrally stable, see example 7.1.

Our main result is theorem 5.1, which gives a set of sufficient conditions for a periodic trajectory near an equilibrium point to be spectrally stable. The examples in $\$ \$ 7$ and 8 show that these conditions are satisfied sufficiently often for the theorem to be useful. Of perhaps wider significance is that in proving this theorem we develop machinery that describes precisely the constraints put on Floquet operators by group actions.

Let $p$ be an equilibrium point, as in the second paragraph above, and let $u(t)$ be a periodic trajectory near $p$ with symmetry group $\Sigma$ and period $T$ near $2 \pi /|\lambda|$, where $\lambda=\lambda_{1}$, say. In $\S 5 a$ we prove that the Floquet operator $\mathrm{M}_{u}: T_{u(0)} \mathrm{P} \rightarrow T_{u(0)} \mathrm{P}$ is close to the operator $\mathrm{e}^{-2 \pi L / \lambda 1}: T_{p} \mathrm{P} \rightarrow$ $T_{p} \mathrm{P}$, which has eigenvalues $\mathrm{e}^{ \pm 2 \pi \lambda_{i} / \lambda \mid}$, on the unit circle. In $\S 5 b$ we use 'Krein theory' to show that if neither $\lambda_{i}$ nor $\lambda_{i}-\lambda_{j}(j \neq i)$ is an integer multiple of $\lambda$ (so that in particular $\mathrm{e}^{ \pm 2 \pi \lambda_{i} / \lambda \mid} \neq 1$ ) then the eigenvalues of $\mathrm{M}_{u}$ near $\mathrm{e}^{ \pm 2 \pi \lambda_{i} / \lambda \mid}$ remain on the unit circle for $u$ near $p$. The eigenvalues of $M_{u}$ near 1 fall into two classes: those that remain at 1 because of the group action, and the rest. Those forced to equal 1 are discussed in $\S 4$. Let $\mathcal{O}=\mathcal{O}_{u}$ as in $(0.1)$. Clearly $\sigma_{T} \mid \mathcal{O}_{u}$ is the identity mapping, so $\mathrm{M}_{u} \mid T_{u(0)} \mathcal{O}_{u}$ is also the identity. More eigenvalues of $\mathrm{M}_{u}$ are forced to equal 1 because the flow $\sigma_{t}$ must leave invariant the momentum mapping, or 'constants of motion', associated to the $\Gamma$-action by Noether's theorem. Theorem 4.5 lets us divide out those parts of $T_{u(0)} \mathrm{P}$ corresponding to eigenvalues of $\mathrm{M}_{u}$ forced to 1 , defining a restricted Floquet operator $\mathrm{N}_{u}$ whose eigenvalues are the remaining eigenvalues of $M_{u}$.

To describe our approach to the eigenvalues of $N_{u}$, we again suppose, for simplicity only, that $\lambda_{1}=\ldots=\lambda_{n}=\lambda$, say. Then $\mathrm{N}_{u}$ is a linear symplectic map which commutes with an action of $\Sigma$ derived from the restriction of the $\Gamma \times \mathbf{S}^{1}$-action on $T_{p} P$. (This is not obvious: see theorem 6.4.) We are therefore able to apply general results about equivariant symplectic maps, proved in $\S 2$. These include a representation-theoretic characterization of cyclospectral representations, i.e. 
representations of $\Sigma$ for which every $\Sigma$-equivariant symplectic linear map has all its eigenvalues on the unit circle (theorem 2.3). This result provides the final ingredient for theorem 5.1. It also has other applications, and in $\S 3$ we apply it to find sufficient conditions for the spectral stability of an equilibrium point. The machinery of $\$ \S 2$ and 4 will also be an essential part of any general discussion of equilibria and periodic trajectories in hamiltonian systems with symmetry.

In the final two sections we illustrate our results on a number of examples. In $\S 7$ we consider equilibria fixed by actions of $Z_{n}, \mathrm{D}_{n}, \mathbf{S O}(2), \mathbf{O}(2), \mathbf{O}(n)$ and $\mathbf{S U}(2)$, showing how results, some of which are perhaps well known, follow from purely group-theoretic considerations. When the symmetry group $\Gamma$ is abelian, the methods of this paper provide no essentially new information on existence or stability, because irreducible symplectic representations are then two dimensional and generically a combination of the standard Liapunov centre theorem and Krein theory yields the same results. However, when $\Gamma$ is not abelian the methods do provide new information, as even the simplest examples show.

In $\S 8$ we look in greater detail at equilibria fixed by actions of $\mathbf{O}(3)$. Near these, under suitable conditions, we prove the existence of a variety of periodic trajectories with prescribed symmetries. These can be approximated by certain linear combinations of rotating spherical harmonics, see figure 6. For low-order spherical harmonics many of these trajectories are forced by the group action to be spectrally stable. As the order increases this is no longer true, although there are always two types of rotating wave that are spectrally stable. However, even here we can use the group action to limit the possible forms that the Floquet operator can take.

An informal discussion of these results, together with further examples, and including applications to vibrations of molecules and crystal lattices, is given in Montaldi et al. (1988a).

\section{EXISTENGE OF PERIODIG SOLUTIONS}

In this section we prove an equivariant version of the Weinstein-Moser theorem on the existence of periodic solutions of a hamiltonian system near an equilibrium (see Weinstein 1973, 1978; Moser 1 976). Suppose that the symmetry group is $\Gamma$, and let $S^{1}$ denote the circle group acting as phase shifts on periodic solutions. The main result, theorem 1.1 below, states that under suitable hypotheses, for each isotropy subgroup $\Sigma$ of a certain action of $\Gamma \times \mathbf{S}^{\mathbf{1}}$, there exist periodic solutions with symmetry group containing $\Sigma$. The number of such solutions is at least half the dimension of the fixed-point subspace of $\Sigma$. We also make a number of remarks on generalizations of the main theorem and related matters, and describe three examples: the spherical pendulum, the Hénon-Heiles system, and the spring pendulum.

We begin by making the setting precise. Let $\mathrm{P}$ be a finite-dimensional symplectic manifold, $\mathscr{H}$ a hamiltonian function on $\mathrm{P}$, and $\mathrm{X}$ its associated vector field, i.e.

$$
\omega(\mathrm{X}(z), v)=\mathrm{D} \mathscr{H}_{z}(v) \quad\left(\forall z \in \mathrm{P}, v \in T_{z} \mathrm{P}\right),
$$

where $\omega$ is the symplectic form on $P$. Suppose that $\Gamma$ is a group acting symplectically on $P$, and $\mathscr{H}$ is $\Gamma$-invariant,

$$
\mathscr{H}(g \cdot z)=\mathscr{H}(z) \quad(\forall g \in \Gamma, z \in \mathrm{P}) .
$$

Then $\mathrm{X}$ is equivariant with respect to the induced action of $\Gamma$ on $T P$,

$$
\mathrm{X}(g \cdot z)=g \cdot \mathrm{X}(z) \quad(\forall g \in \Gamma, z \in \mathrm{P}) .
$$


Let $p \in \mathrm{P}$ be a critical point of $\mathscr{H}$, hence an equilibrium point of $\mathrm{X}$, and suppose that $p$ is fixed by $\Gamma$.

Let $\mathscr{C}^{k}(T)$ denote the space of continuously $k$-times differentiable $T$-periodic maps $u: \mathbb{R} \rightarrow \mathrm{P}$. This space will, of course, contain all $T$-periodic trajectories of X. Identify the circle group $\mathbf{S}^{1}$ with $\mathbb{R} / 2 \pi \mathbb{Z}$ and define an action of $\Gamma \times \mathbf{S}^{1}$ on $\mathscr{C}^{k}(T)$ by

for all $\gamma \in \Gamma, \theta \in \mathbf{S}^{1}, u \in \mathscr{C}^{k}(T)$.

$$
(\gamma, \theta) \cdot u(s)=\gamma u(s+T \theta / 2 \pi)
$$

Recall that for any action of a group $\mathrm{G}$ on a set $\mathrm{Y}$ the isotropy subgroup $\mathrm{G}_{y}$ of a point $y \in \mathrm{Y}$ is defined by

$$
\mathrm{G}_{y}=\{\sigma \in \mathrm{G}: \sigma \cdot y=y\}
$$

and that for any subgroup $\Sigma$ of $\mathrm{G}$ the fixed-point set Fix $(\Sigma ; \mathrm{Y})$ is defined by

$$
\operatorname{Fix}(\Sigma ; \mathrm{Y})=\{y \in \mathrm{Y}: \sigma \cdot y=y \text { for all } \sigma \in \Sigma\} .
$$

We write Fix $(\Sigma)$ for this if there is no ambiguity. When G acts linearly, Fix $(\Sigma)$ is a linear subspace.

Let $u$ be a $T$-periodic trajectory of $\mathrm{X}$, or indeed of any equivariant vector field, and consider $u$ as an element of $\mathscr{C}^{k}(T)$ with the above $\Gamma \times \mathbf{S}^{1}$-action. The isotropy subgroup of $u$ is called the symmetry group of $u$. Note that this definition depends on specifying the period $T$ of $u$, which may not be minimal. In fact $u$ has minimal period $T / k$ if and only if $\Sigma_{u} \cap \mathbf{S}^{1}=\mathbf{Z}_{k}$, the finite cyclic group of order $k$.

Below we state a theorem on the existence of $T$-periodic trajectories near $p$, with prescribed symmetry group. This theorem will be proved by considering $\mathrm{X}$ as a perturbation of its linearization at $p$, the trajectories of which are the solutions of

$$
\dot{x}+\mathrm{L} x=0,
$$

where $\mathrm{L}=\mathrm{DX}_{p}$. The equivariance of $\mathrm{X}$ implies that $\mathrm{L}$ is a $\Gamma$-equivariant linear transformation of $\mathrm{V}=T_{p} P$. Let $\lambda$ be a non-zero purely imaginary eigenvalue of $\mathrm{L}$, and define the resonance space $\mathrm{V}_{\lambda}$ for $\lambda$ to be the unique subspace of $\mathrm{V}$, maximal with respect to all eigenvalues of $L \mid \mathrm{V}_{\lambda}$ being integer multiples of $\lambda$. In other words, $V_{\lambda}$ is the real part of the sum of the generalized eigenspaces of $\mathrm{L}$ for eigenvalues $k \lambda, k \in \mathbb{Z}$. Assume the following conditions on $\mathscr{H}$.

$\mathrm{H1}: \mathrm{D}^{2} \mathscr{H}_{p}$ is a non-degenerate quadratic form;

$\mathrm{H} 2: \mathrm{D}^{2} \mathscr{H}_{p} \mid \mathrm{V}_{\lambda}$ is positive definite.

The condition $\mathrm{H} 1$ is equivalent to $L$ being non-singular; and $\mathrm{H} 2$ implies that $L \mid \mathrm{V}_{\lambda}$ is semisimple, that is, can be diagonalized over $\mathbb{C}$.

The $\Gamma$-equivariance of $L$ implies that $V_{\lambda}$ is invariant under the action of $\Gamma$ on $V$. It is clear that $V_{\lambda}$ is also invariant under the flow obtained by integrating (1.2). Because $L \mid V_{\lambda}$ is semisimple, the trajectories of this flow on $V_{\lambda}$ are all periodic with period $2 \pi /|\lambda|$ and so define an action of $\mathbf{S}^{1}$. This action of $\mathbf{S}^{1}$ commutes with that of $\Gamma$ and so together they define an action of $\Gamma \times \mathbf{S}^{1}$.

The $\mathbf{S}^{1}$-action can be described explicitly as follows. Let the eigenvalues of $L \mid V_{\lambda}$ be $\pm m_{j} \lambda$, where $m_{1}, \ldots, m_{n}$ are strictly positive integers. Then $\mathrm{V}_{\lambda}$ can be identified with $\mathbb{C}^{n}$ and $\mathbf{S}^{1}$ with the complex numbers $\mathrm{e}^{\mathrm{i} \psi}, 0 \leqslant \psi<2 \pi$, acting by

$$
\mathrm{e}^{\mathrm{i} \psi}\left(z_{1}, \ldots, z_{n}\right)=\left(\mathrm{e}^{\mathrm{i} m_{1} \psi} z_{1}, \ldots, \mathrm{e}^{\mathrm{i} m_{n} \psi} z_{n}\right) .
$$

The periodic trajectories of $\mathrm{X}$, whose existence will be proved below, are obtained by perturbing 
periodic solutions of (1.2) lying in $V_{\lambda}$. An essential point of the theorem is that symmetry groups are preserved by these perturbations.

Theorem 1.1. (Equivariant Weinstein-Moser theorem.) Suppose the hamiltonian $\mathscr{H}$ satisfies $\mathbf{H} 1$ and H2. Then for every isotropy subgroup $\Sigma$ of the $\Gamma \times \mathbf{S}^{1}$-action on $\mathrm{V}_{\lambda}$, and for all $\epsilon$ sufficiently small, there exist at least

$$
\frac{1}{2} \operatorname{dim} \operatorname{Fix}\left(\Sigma, V_{\lambda}\right)
$$

periodic trajectories of $\mathrm{X}$, with periods near $2 \pi /|\lambda|$ and symmetry group containing $\Sigma$, on the energy surface $\mathscr{H}(x)=\mathscr{H}(p)+\epsilon^{2}$.

Note that by taking $\Gamma$ to be trivial and $\Sigma$ to be the trivial isotropy subgroup we recover the Weinstein-Moser theorem. Our proof of theorem 1.1 is an equivariant adaptation of Weinstein (1978).

Proof. Because the theorem is local we may, without loss of generality, identify $\mathbf{P}$ and the $\Gamma$ action in a neighbourhood of $p$ with $\mathrm{V}=T_{p} \mathrm{P}$ and its $\Gamma$-action. This follows from an equivariant version of Darboux's theorem (Guillemin \& Sternberg 1984), theorem 22.2. The point $p$ becomes the origin in $\mathrm{V}$. We may also suppose $\mathscr{H}(0)=0$. The trajectories of $\mathrm{X}$ are solutions of the differential equation

$$
\dot{x}+\mathrm{X}(x)=0 \text {. }
$$

Writing $x=\epsilon y$ and dividing by $\epsilon(1.3)$ becomes

$$
\dot{y}+\mathrm{L} y+\epsilon \overline{\mathrm{X}}(y)=0
$$

where $\overline{\mathrm{X}}(y)=\epsilon^{-2}[\mathrm{X}(\epsilon y)-\epsilon \mathrm{L} y]$ is a smooth $\Gamma$-equivariant vector field. Equation (1.4) has $\overline{\mathscr{H}}(y)=\epsilon^{-2} \mathscr{H}(\epsilon y)$ as a hamiltonian function. So $T$-periodic solutions of $(1.3)$ with energy $\epsilon^{2}$ correspond precisely to $T$-periodic solutions of (1.4) with energy equal to 1 .

For $\epsilon=0$, the $2 \pi /|\lambda|$-periodic solutions of (1.4) are precisely the trajectories of the linearized equation (1.2) that lie in $V_{\lambda}$. This gives a $\Gamma \times S^{1}$-equivariant embedding of $V_{\lambda}$ into $\mathscr{C}^{k}(2 \pi /|\lambda|)$. With this identification, the $2 \pi /|\lambda|$-periodic solutions with energy 1 correspond to the points on an ellipsoid $S$ in $V_{\lambda}$. We claim that $S$ is a non-degenerate periodic manifold for (1.3) with $\epsilon=0$, in the sense of Weinstein (1978). This is easily checked by the corollary on p. 246 of that paper.

Theorems on pp. 241 and 247 of Weinstein (1978) imply that for any neighbourhood $U$ of $\mathbf{S} \times\{2 \pi /|\lambda|\}$ in $\mathscr{C}^{k}(2 \pi /|\lambda|) \times \mathbb{R}$ there exists $\epsilon_{0}>0$ such that for $|\epsilon|<\epsilon_{0}$ there is an embedding $e_{\varepsilon}$ of $\mathbf{S}$ in $\mathrm{U}$, and a smooth function $A_{\varepsilon}$ on $e_{\epsilon}(\mathbf{S})$, such that the critical points $(u, T)$ of $A_{\varepsilon}$ are precisely the pairs in $\mathrm{U}$ given by $T$-periodic solutions of $(\mathbf{1 . 3})$ with energy equal to 1 .

The submanifold $S$ is invariant under the $\Gamma \times S^{1}$-action on $\mathscr{C}^{k}(2 \pi /|\lambda|)$. Remark 1 on p. 243 of Weinstein (1978) implies that $e_{\varepsilon}$ is equivariant with respect to this action (extended to be trivial on the factor $\mathbb{R}$ in $\left.\mathscr{C}^{k}(2 \pi /|\lambda|) \times \mathbb{R}\right)$, and $A_{\epsilon}$ is invariant under the induced action on $e_{\varepsilon}(\mathbf{S})$.

Let $\Sigma$ be an isotropy subgroup of the $\Gamma \times \mathbf{S}^{1}$-action on $\mathrm{V}_{\lambda}$, and hence also for its induced action on $\mathbf{S}$. Then the critical points of $A_{\epsilon}$ fixed by $\Sigma$ are precisely the critical points of the restriction of $A_{\epsilon}$ to Fix $\left(\Sigma ; e_{\epsilon}(\mathbf{S})\right)$, by the principle of symmetric criticality, see Palais (1979). This fixed-point set is diffeomorphic to a sphere; moreover, because the $\mathbf{S}^{\mathbf{1}}$-action commutes with that of $\Gamma$, it restricts to an action on Fix $\left(\Sigma ; e_{\varepsilon}(\mathbf{S})\right)$ which leaves the restriction of $A_{\epsilon}$ invariant. It now follows by theorem 1.3 of Weinstein (1973) that $A_{\epsilon}$ must have at least

$$
\frac{1}{2}\left[\operatorname{dim} \operatorname{Fix}\left(\Sigma ; e_{\varepsilon}(\mathbf{S})\right)+1\right]=\frac{1}{2} \operatorname{dim} \operatorname{Fix}\left(\Sigma, \mathrm{V}_{\lambda}\right)
$$

$\mathbf{S}^{1}$-orbits of critical points fixed by $\boldsymbol{\Sigma}$. 


\section{Remarks 1.2}

(a) If $\Sigma$ is maximal (with respect to inclusion) among isotropy subgroups of non-zero points in $\mathrm{V}_{\lambda}$, then we can make the slightly stronger statement that there are at least $\frac{1}{2} \operatorname{dim} \mathrm{Fix}\left(\Sigma ; \mathrm{V}_{\lambda}\right)$ periodic trajectories with symmetry group equal to $\Sigma$, on each level for a sufficiently small positive energy.

(b) By a closer examination of the proofs of the Weinstein-Moser theorem it can be shown that if $\operatorname{dim} \operatorname{Fix}\left(\Sigma ; \mathrm{V}_{\lambda}\right)=2$ (an important special case in which $\Sigma$ is necessarily maximal) then through $p$ there is a $C^{\infty}$ two-dimensional submanifold of $\mathrm{P}$ that is foliated by periodic trajectories with periods near $2 \pi /|\lambda|$ and symmetry groups $\Sigma$. The tangent space to this submanifold at $p$ is equal to Fix $\left(\Sigma ; V_{\lambda}\right)$. This is an equivariant generalization of the Liapunov centre theorem (Abraham \& Marsden 1978 theorem 5.6.7). For higher-dimensional fixed-point spaces we expect the periodic trajectories to form families that are $C^{\infty}$ away from the origin, but have conelike singularities at the origin. These families lie inside submanifolds of $\mathbf{P}$ that are $C^{k}$ at $p$ for any finite $k$, and have tangent spaces at $p$ equal to Fix $\left(\Sigma ; V_{\lambda}\right)$. A similar situation is treated in Duistermaat ( 1984 ).

(c) It can also be shown that $\mathrm{H} 2$ may be relaxed to the condition that $\mathrm{L} / \mathrm{V}_{\lambda}$ is semisimple and $\mathrm{D}^{2} \mathscr{H}_{p} \mid \mathrm{Fix}\left(\Sigma ; \mathrm{V}_{\lambda}\right)$ is positive definite.

(d) Suppose, for simplicity, that $\mathrm{V}_{\lambda}=\mathrm{V}=\mathrm{P}$. Then the hamiltonian $\mathscr{H}$ of theorem 1.1 is said to be in Birkhoff normal form if it is invariant under the action of $\mathbf{S}^{\mathbf{1}}$ defined by the linearization of $\mathrm{X}$ at $p$. This can always be arranged, by an equivariant symplectic change of coordinates, to any finite degree in the Taylor series of $\mathscr{H}$ about $p$ (see, for example, Churchill et al. 1983), but the resulting formal power series is not, in general, convergent.

If $\mathscr{H}$ is in Birkhoff normal form then the proof of theorem 1.1 follows directly from the Weinstein-Moser theorem together with the observation that for each $\Sigma$ the restricted vector field $\mathrm{X} \mid \mathrm{Fix}(\Sigma ; \mathrm{V})$ is tangent to Fix $(\Sigma ; \mathrm{V})$ and has $\mathscr{H} \mid \mathrm{Fix}(\Sigma ; \mathrm{V})$ as hamiltonian. The periodic trajectories of $\mathrm{X}$ that are fixed by $\Sigma$ are precisely the periodic trajectories of $X \mid \mathrm{Fix}(\Sigma ; \mathrm{V})$, and so the theorem follows from the Weinstein-Moser theorem applied to this vector field.

(e) The previous result is related to the approach of Churchill et al. (1983) to periodic orbits in symmetric hamiltonian systems. Suppose that $\mathrm{G}$ is a compact Lie group acting symplectically on $\mathrm{P}$ and let $\Phi: \mathrm{P} \rightarrow \mathrm{g}^{*}$ be the associated momentum map (see $\S 4$ and references therein). Suppose $\mu \in \mathrm{g}^{*}$ is a regular value of $\Phi$. Then $\Phi^{-1}(\mu)$ is a smooth manifold on which $\mathrm{G}_{\mu}$ (the isotropy group of the co-adjoint action of $\mathrm{G}$ on $\mathrm{g}^{*}$ at $\mu$ ) acts. The smooth part $\mathrm{Q}$ of the quotient $\Phi^{-1}(\mu) / G_{\mu}$ inherits a symplectic form from $P$. This is the Marsden-Weinstein reduced phase space (see Abraham \& Marsden 1978; Arnold 1978). If X is a G-equivariant hamiltonian vector field on $\mathrm{P}$ then there is an induced hamiltonian vector field $\mathrm{Y}$ on $\mathrm{Q}$ such that trajectories of $\mathrm{X}$ in $\Phi^{-1}(\mu)$ project to trajectories of $Y$ on $Q$. In particular, equilibrium points of $Y$ correspond to $\mathrm{G}_{\mu}$-orbits in $\Phi^{-1}(\mu)$ that are invariant under the flow generated by $\mathrm{X}$. These are the relative equilibria of $\mathrm{X}$ with respect to the $\mathrm{G}$-action. If $\mathrm{G}=\mathbf{S}^{\mathbf{1}}$ then relative equilibria are periodic trajectories. Conversely, if $\mathrm{V}=\mathrm{V}_{\lambda}=\mathrm{P}$ and $\mathscr{H}$ is in Birkhoff normal form as in remark 4, then the periodic trajectories near $p$ with periods near $2 \pi /|\lambda|$ are the relative equilibria of $\mathrm{X}$ with respect to the $\mathbf{S}^{1}$-action on $\mathrm{V}$, provided that $\mathrm{Q}$ is smooth at the appropriate point.

Now suppose that $\Gamma$ is another compact group acting symplectically on $P$, that this action commutes with that of $\mathrm{G}$, and that $\mathrm{X}$ is $\Gamma$-equivariant as well as $\mathrm{G}$-equivariant. Then there is an induced action of $\Gamma$ on $Q$ with respect to which $Y$ is equivariant. Any isolated fixed point 
$q \in \mathrm{Q}$ of this action must be an equilibrium point of $\mathrm{Y}$ and hence a relative equilibrium of $\mathrm{X}$. There may, of course, be others.

Churchill et al. use these ideas to study hamiltonian vector fields on $\mathbb{R}^{4}$, with separate arguments to deal with the singular points of $Q$ and to show that periodic trajectories of the Birkhoff normal form persist as periodic solutions of the original system, sufficiently close to the equilibrium point.

$(f)$ Fadell \& Rabinowitz (1978) have shown that (roughly) the number of periodic trajectories with period near $2 \pi /|\lambda|$, in hamiltonian systems that do not satisfy $\mathrm{H} 2$, but for which $\mathrm{L} \mid \mathrm{V}_{\lambda}$ is still semisimple, is at least $\frac{1}{2}|\nu|$ where $\nu$ is the signature of $\mathrm{D}^{2} \mathscr{H}_{p} \mid \mathrm{V}_{\lambda}$. In these circumstances the flow of the linearized equations still defines an $\mathbf{S}^{\mathbf{1}}$-action on $\mathrm{V}_{\lambda}$, and in a $\Gamma$ equivariant system there will also be a $\Gamma$-action. We conjecture that if $\Sigma$ is an isotropy subgroup of the $\Gamma \times \mathbf{S}^{1}$-action on $V_{\lambda}$, then the number of periodic trajectories with period near $2 \pi /|\lambda|$ and symmetry groups containing $\Sigma$ will be at least

$$
\frac{1}{2}\left|\operatorname{sgn} D^{2} \mathscr{H}_{p}\right| \operatorname{Fix}\left(\Sigma ; \mathrm{V}_{\lambda}\right) \mid .
$$

(g) We can also prove an equivariant version of the result of Moser (1976) for conservative systems. We continue to suppose that $\mathrm{L}=\mathrm{DX}_{p}$ is non-singular, but now take $\mathscr{H}$ to be only a conserved quantity satisfying $\mathrm{H} 2$. With these hypotheses theorem 1.1 remains true, except that we can guarantee only one periodic trajectory fixed by $\Sigma$, no matter what the dimension of Fix $(\Sigma)$ is. The equivariant Liapunov centre theorem also continues to hold in this case.

(h) Following Schmidt (1976) and Alexander \& Yorke (1978), Hopf bifurcation theorems can be used to prove the existence of periodic solutions near equilibria of hamiltonian and conservative systems. In the same way, equivariant Hopf bifurcation theorems, such as those in Golubitsky \& Stewart (1985) and Fiedler (1986), may be applied to symmetric hamiltonian and conservative systems. For hamiltonian systems the conclusions obtained in this manner are decidedly weaker than theorem 1.1. For conservative systems the results are essentially equivalent to those in remark $(g)$.

The calculations needed to apply theorem 1.1 to specific systems, namely the computation of isotropy subgroups of the $\Gamma \times \mathbf{S}^{1}$-action on $V_{\lambda}$, are identical to those needed to apply the equivariant Hopf bifurcation theorems of Golubitsky \& Stewart (1985) and Fiedler (1986). This will be clear in the examples below.

(i) Theorem 1.1 is an existence result. For an explicitly given hamiltonian, perturbation series expansions for the periodic orbits near an equilibrium point can, at least in principle, be calculated by using Liapunov-Schmidt reduction and Birkhoff normal form. Examples will be given in (Montaldi et al. 1988b).

\section{Examples 1.3}

(a) The spherical pendulum. The spherical pendulum is symmetric with respect to rotations about the equilibrium position and reflections in vertical planes containing the support. Thus the symmetry group is $\mathbf{O}(2)$. The stable vertical equilibrium position is fixed by $\mathbf{O}(2)$; we can identify the tangent space $\mathrm{V}$ to the phase space at this point with $\mathbb{C}^{2}$. The group $\mathbf{O}(2)$ is generated by its subgroup of rotations, $\mathrm{SO}(2)=\{\theta: 0 \leqslant \theta<2 \pi\}$ and an arbitrary reflection $\kappa$. We show in $\S 7$ below that the action on $\mathrm{V} \equiv \mathbb{C}^{2}$ can be written as

$$
\begin{aligned}
& \theta\left(z_{1}, z_{2}\right)=\left(\mathrm{e}^{\mathrm{i} \theta} z_{1}, \mathrm{e}^{-1 \theta} z_{2}\right), \\
& \kappa\left(z_{1}, z_{2}\right)=\left(z_{2}, z_{1}\right) .
\end{aligned}
$$


The eigenvalues of the linearization about the stable equilibrium are imaginary, and are forced to be double by the group action. The $\mathbf{S}^{\mathbf{1}}$-action defined by the linear flow is

$$
\phi\left(z_{1}, z_{2}\right)=\left(\mathrm{e}^{1 \phi} z_{1}, \mathrm{e}^{1 \phi} z_{2}\right)
$$

This is identical to the group action discussed in Golubitsky \& Stewart (1985) in the context of Hopf bifurcation with $\mathbf{O}(2)$ symmetry, although their coordinate system is different. From that paper the maximal isotropy subgroups are conjugate either to

$$
\widetilde{\mathbf{S O}}(2)=\left\{(\theta,-\theta) \in \mathbf{S O}(2) \times \mathbf{S}^{\mathbf{1}}\right\}
$$

or to

$$
\mathbf{Z}_{2} \oplus \mathbf{Z}_{2}^{c}
$$

where

$$
\begin{aligned}
Z_{2} & =\{1, \kappa\}, \\
Z_{2}{ }^{c} & =\left\{1,(\pi, \pi) \in \mathbf{S O}(2) \times \mathbf{S}^{1}\right\} .
\end{aligned}
$$

The group $\mathbf{Z}_{2}{ }^{c}$ acts trivially on $\mathbb{C}^{2}$.

Each maximal isotropy group has a two-dimensional fixed-point space. By theorem 1.1, on each energy level near the equilibrium, there is a periodic trajectory with each of these symmetry groups. Other periodic trajectories with conjugate symmetry groups are obtained by applying elements of $\Gamma=\mathbf{O}(2) \times \mathbf{S}^{1}$ to these. In total there are two periodic trajectories with symmetry groups conjugate to $\widetilde{\mathbf{S O}}(2)$, and an infinite family, foliating an invariant torus, with symmetry groups conjugate to $\mathbf{Z}_{2} \oplus \mathbf{Z}_{2}{ }^{c}$. The former are the periodic trajectories where the pendulum rotates in a horizontal circle (one clockwise trajectory, one anti-clockwise). The latter are the trajectories where the pendulum swings in a fixed vertical plane, and there is an infinite family of such planes. These are in fact the only periodic trajectories 'near' those of the linearized system.

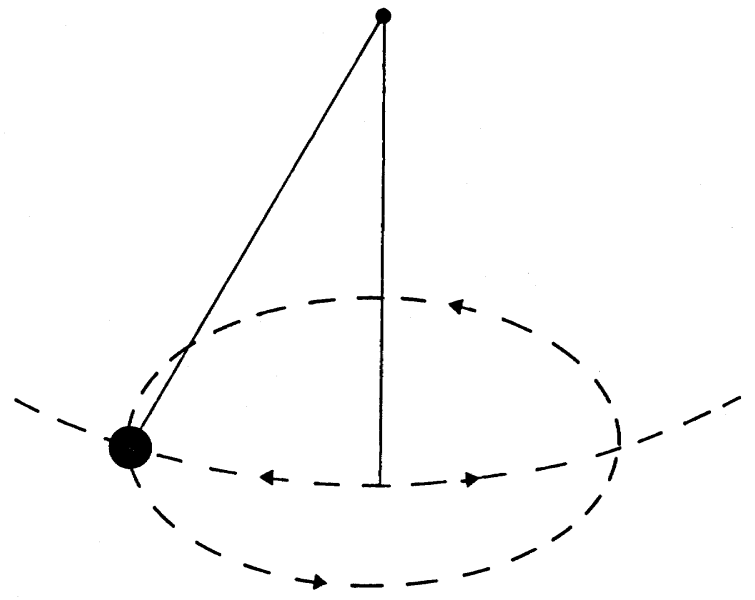

FIGURE 1. Circular and planar trajectories of the spherical pendulum.

(b) The Hénon-Heiles hamiltonian. For details see Hénon \& Heiles (1964) and Rod \& Churchill (1985). Let $\Gamma=D_{3}$, the dihedral group of order 6 (or symmetry group of an equilateral triangle). Identify this with the subgroup of $\mathbf{O}(2)$ generated by $\frac{2}{3} \pi \in \mathbf{S O}(2)$ and $\kappa$, by using the notation of example $1.3 a$ and let it act on $\mathrm{P}=\mathrm{V}=\mathbb{C}^{2}$ by the restriction of the $\mathbf{O}(2)$-action above. Let $\mathscr{H}$ be a hamiltonian on $\mathbb{C}^{2}$ that is invariant under this action; an example is the Hénon-Heiles hamiltonian. Again the group action forces the eigenvalues of the linearization 
to be double, and we suppose they are imaginary as in the Hénon-Heiles system. The $\mathbf{S}^{\mathbf{1}}$-action given by the linearization is the same as in example $1.3 a$. The maximal isotropy subgroups of the combined $\mathbf{D}_{3} \times \mathbf{S}^{1}$-action are calculated in Golubitsky \& Stewart (1986). They are conjugate to one of the following subgroups:

where $\pi \in \mathbf{S}^{\mathbf{1}}$.

$$
\begin{aligned}
\tilde{\mathbf{Z}}_{3} & =\left\{(\theta,-\theta) \in \mathrm{D}_{3} \times \mathbf{S}^{1}: \theta=0, \frac{2}{3} \pi, \frac{4}{3} \pi\right\}, \\
\mathbf{Z}_{2}{ }^{\kappa} & =\{1, \kappa\}, \\
\mathbf{Z}_{2}{ }^{(\kappa, \pi)} & =\{1,(\kappa, \pi)\},
\end{aligned}
$$

Again each maximal isotropy subgroup has a two-dimensional fixed-point space, so (by applying the $\mathbf{D}_{3} \times \mathbf{S}^{1}$-action) theorem 1.1 yields two periodic trajectories with symmetry groups conjugate to $\tilde{\mathbf{Z}}_{3}$ and three periodic trajectories with symmetry groups conjugate to each of $\mathbf{Z}_{2}{ }^{\kappa}, \mathbf{Z}_{2}{ }^{(\kappa, \pi)}$. These are the only periodic trajectories of the Hénon-Heiles system near periodic trajectories of the linearization. We can identify these eight solutions with those shown in figure 2, taken from Churchill et al. (1983), as follows:

$$
\begin{aligned}
& \Pi_{1}, \Pi_{2}, \Pi_{3} \text { have symmetry groups conjugate to } Z_{2}{ }^{k} \text {. } \\
& \Pi_{4}, \Pi_{5}, \Pi_{6} \text { have symmetry groups conjugate to } Z_{2}{ }^{(\kappa, \pi)} \text {. } \\
& \Pi_{7}, \Pi_{8} \text { have symmetry groups conjugate to } \tilde{Z}_{3} \text {. }
\end{aligned}
$$

These results are generalized to systems with $\mathbf{D}_{n}$ symmetry in $\S 7$.

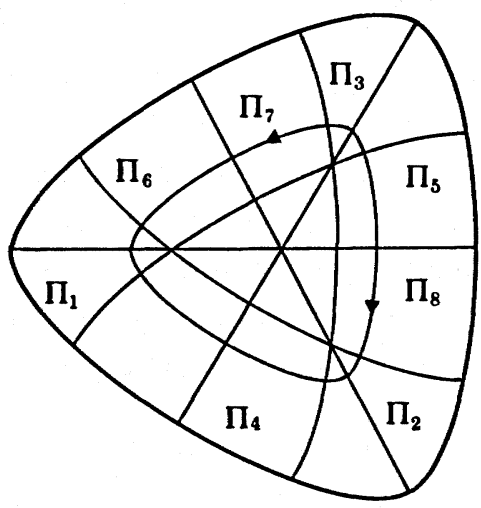

Figure 2. Periodic trajectories in the Hénon-Heiles system, after Churchill et al. (1983).

(c) Spring pendulum. Consider a pendulum in a vertical plane suspended by a spring instead of a stiff rod. This is a hamiltonian system invariant under reflection through the vertical line. In a neighbourhood of the vertical equilibrium position (that minimizes the hamiltonian) the phase space is isomorphic to $\mathbb{C}^{2}$ with $\mathbf{Z}_{2}=\{1, \kappa\}$ acting by $\kappa \cdot\left(z_{1}, z_{2}\right)=\left(-z_{1}, z_{2}\right)$. The eigenvalues of the linearization about the vertical equilibrium are imaginary, but vary with the spring constant, the equilibrium length of the pendulum, etc. In particular they may be resonant (for details see Duistermaat 1984). In all cases the Weinstein-Moser theorem guarantees at least two periodic trajectories in each energy level near equilibrium, with periods near those of the linearized system.

If the eigenvalues $\pm \lambda_{1}, \pm \lambda_{2}$ are non-resonant then the resonance spaces $V_{\lambda_{1}}$ are both two dimensional and can be identified with $\mathbb{C}$. On one, say $V_{\lambda_{1}}$, the flip $\kappa$ acts trivially, whereas on 
the other it acts non-trivially. On each, $\mathbf{S}^{\mathbf{1}}$ acts as multiplication by unit-circle complex numbers. With respect to these actions of $Z_{2} \times \mathbf{S}^{1}$, the space $V_{\lambda_{1}}$ is fixed pointwise by $Z_{2}{ }^{k}$, whereas $\mathrm{V}_{\lambda_{2}}$ is fixed pointwise by $\mathrm{Z}_{2}{ }^{\kappa, \pi}=\{1,(\kappa, \pi)\}$. Thus theorem 1.1 implies that on each energy level near the stable equilibrium there exists one periodic trajectory fixed by $\kappa$, with period near $2 \pi /\left|\lambda_{1}\right|$, and another fixed by $(\kappa, \pi)$ with period near $2 \pi /\left|\lambda_{2}\right|$.

Suppose now that $\lambda_{1}=\lambda_{2}=\lambda$. Then $V_{\lambda}=\mathbb{C}^{2}$ with action of $Z_{2}$ as above, and again $\mathbf{S}^{1}$ acts as multiplication by unit-circle complex numbers. The non-trivial isotropy subgroups are $\mathbf{Z}_{2}{ }^{*}$ and $Z_{2}{ }^{(\kappa, \pi)}$, defined as above, both with two-dimensional fixed-point subspaces. Again theorem 1.1 implies that there exists one periodic trajectory fixed by $\kappa$ and one fixed by $(\kappa, \pi)$, both with period near $2 \pi /|\lambda|$.

In fact, by applying theorem 1.1 to the possible eigenspaces $V_{\lambda}$ that can occur in both nonresonant and resonant equilibria, it can be shown that there is always one periodic trajectory with $\kappa$ in its symmetry group, and another with $(\kappa, \pi) \in \mathbf{Z}_{2} \times \mathbf{S}^{\mathbf{1}}$ in its symmetry group, provided that both symmetry groups are taken with respect to the minimal periods of the trajectories. These may be thought of as spring-like and pendulum-like trajectories respectively. We do not obtain more periodic orbits than the Weinstein-Moser theorem gives; but we derive extra information about their symmetries.

\section{EQUIVARIANT SYMPLEGTIC LINEAR MAPPINGS}

A symplectic representation of a group $G$ is a real vector space $V$ on which is defined a symplectic form $[$,$] and a linear action of G$ that preserves the symplectic form:

$$
[g \cdot v, g \cdot w]=[v, w] \quad(v, w \in \mathrm{V}, g \in \mathrm{G}) .
$$

Recall that an equivariant mapping $\mathrm{V} \rightarrow \mathrm{V}$ is one that commutes with the actions of all $g \in \mathrm{G}$. A symplectic representation $\mathrm{V}$ is said to be cyclospectral if every equivariant symplectic linear mapping $\mathrm{N}: \mathrm{V} \rightarrow \mathrm{V}$ has all its eigenvalues on the unit circle. The main aim of this section is to characterize cyclospectral representations of compact groups in a computable manner. We state the main theorems in $\S 2 a$, illustrating them on the group $\mathbf{S}^{1}$. The proofs, which are technical, are given in $\S 2 b-d$.

\section{(a) Statement of results}

We begin by recalling some standard facts about representations of a compact Lie group G. See Adams (1969) for further details.

A representation of $\mathrm{G}$ is irreducible if it has no proper non-zero invariant subspace. If $\mathrm{V}$ is a representation of $G$ we write $\operatorname{Hom}_{G}(V, V)$ for the set of equivariant linear maps $V \rightarrow V$. If $W$ is an irreducible representation then $\operatorname{Hom}_{G}(W, W)$ is a real division algebra (with multiplication given by composition of maps), hence isomorphic to one of $\mathbb{R}, \mathbb{C}$, or $\mathbb{H}$. We say that $\mathrm{W}$ is real, complex, or quaternionic according to which possibility is realized.

An arbitrary representation $\mathrm{V}$ can be decomposed as a direct sum of irreducible representations. By collecting together all summands of a given isomorphism type we obtain a unique isotypic decomposition

$$
\mathrm{V}=\mathrm{V}_{1} \oplus \ldots \oplus \mathrm{V}_{l}
$$

where each $V_{j}$ is a direct sum of subspaces, each isomorphic to a single irreducible representation $\mathrm{W}_{j}$, such that if $j \neq k$ then $\mathrm{W}_{j}$ is not isomorphic to $\mathrm{W}_{k}$. If $\mathrm{W}_{j}$ is a real, complex or 
quaternionic irreducible representation then we refer to $V_{j}$ as a real, complex or quaternionic isotypic block, respectively.

If $\mathrm{V}$ is a symplectic representation then by ignoring the symplectic structure we obtain an ordinary representation, which we call the underlying representation of $\mathrm{V}$. An irreducible symplectic representation is one that has no proper non-zero invariant symplectic subspaces. The underlying representation need not be irreducible in this case: the first result describes the relation in detail.

Theorem 2.1. (Classification of irreducible symplectic representations.)

(a) The underlying representation of an irreducible symplectic representation is either:

(i) a complex or quaternionic irreducible representation, or

(ii) isomorphic to $\mathrm{W} \oplus \mathrm{W}$ where $\mathrm{W}$ is a real irreducible representation.

(b) In the real and quaternionic cases the isomorphism type of the irreducible symplectic representation is uniquely determined by that of its underlying representation.

(c) In the complex case there are precisely two isomorphism types of irreducible symplectic representation for a given complex irreducible underlying representation.

The two types of irreducible symplectic representation that occur for a given complex irreducible underlying representation are dual to each other, in a sense to be made precise in $\S 2 b$.

An irreducible symplectic representation is referred to as real, complex, or quaternionic according to the type of its underlying representation.

Example. Let $\mathrm{V}=\mathbb{C}$ with symplectic form

$$
[z, w]=\operatorname{Im} z \bar{w}
$$

Let $\mathrm{G}=\mathbf{S}^{1}$, identified with the unit complex numbers $\left\{\mathrm{e}^{\mathbf{1} \theta}\right\}$. For every integer $r$ there is a symplectic action $\sigma_{r}$ of $\mathrm{G}$ on $\mathrm{V}$ given by

$$
\mathrm{e}^{\mathrm{i} \theta} \cdot z=\mathrm{e}^{\mathrm{r} \theta} z
$$

If $r \neq 0$ this is a complex irreducible symplectic representation; if $r=0$ it is a real irreducible symplectic representation. If $r \neq s$ then $\sigma_{r}$ and $\sigma_{s}$ are not isomorphic as symplectic representations. However, their underlying representations are isomorphic if $r=-s$, the isomorphism being $z \mapsto \bar{z}$. Every irreducible symplectic representation of $\mathbf{S}^{\mathbf{1}}$ is isomorphic to some $\sigma_{r}$.

TheOREM 2.2. (Isotypic decomposition of symplectic representations.) Every symplectic representation $\mathrm{V}$ has a unique direct sum decomposition

$$
\mathrm{V}=\mathrm{V}_{1} \oplus \ldots \oplus \mathrm{V}_{\ell}
$$

where each $\mathrm{V}_{\text {, }}$ is a symplectic subspace of $\mathrm{V}$, and either

(a) $\mathrm{V}_{\mathrm{j}}$ is isomorphic to a direct sum of a number of copies of a single real or quaternionic irreducible symplectic representation, or

(b) $\mathrm{V}_{j}$ is isomorphic to a direct sum

$$
\left(\oplus^{s} \mathrm{X}\right) \oplus\left(\oplus^{t} \mathrm{Y}\right) \quad(s \geqslant 0, t \geqslant 0),
$$

where $\mathrm{X}$ and $\mathrm{Y}$ are a (dual) pair of complex irreducible symplectic representations whose underlying representations are isomorphic.

Example. There is a bijection between isomorphism classes of symplectic representations $\mathrm{V}$ of $\mathbf{S}^{1}$ and sequences of integers $\left(r_{1}, \ldots, r_{k}\right)$ with $r_{1} \leqslant \ldots \leqslant r_{k}$. Namely, decompose the 
representation into a direct sum of $\sigma_{r} s$ and list the $r s$ that occur in ascending order (with appropriate multiplicity). Let $\left(R_{1}, \ldots, R_{\ell}\right)$ be the distinct values of $\left|r_{j}\right|$ listed in ascending order. Then the isotypic decomposition of $\mathrm{V}$ has the form

$$
\mathrm{V}=\bigoplus_{j=1}^{\ell} \mathrm{V}_{j}
$$

where $\mathrm{V}_{j}$ is the sum of the irreducible subspaces of $\mathrm{V}$ on which $\mathbf{S}^{1}$ acts either by $\sigma_{R_{j}}$ or $\sigma_{-R_{j}}$.

THEOREM 2.3. (Gharacterization of cyclospectral representations.) A symplectic representation $\mathrm{V}$ is cyclospectral if and only if, in its isotypic decomposition:

(a) there are no real isotypic blocks;

(b) no complex isotypic block contains two distinct complex irreducible symplectic representations (necessarily dual to each other);

(c) every quaternionic isotypic block consists of a single quaternionic irreducible representation.

More briefly: 'cyclospectral' means 'no reals, no dual complex pairs, no multiple quaternionics'.

Example. There is a bijection between isomorphism classes of cyclospectral symplectic representations of $\mathbf{S}^{1}$ and sequences of integers $\left(r_{1}, \ldots, r_{k}\right)$ with $r_{1} \leqslant \ldots \leqslant r_{k}$ such that $r$ and $-r$ do not both occur in the sequence (so in particular 0 does not occur). Proof: there are no quaternionic irreducible representations of $\mathbf{S}^{\mathbf{1}}$. There is a real block if and only if $\sigma_{0}$ occurs. Complex dual pairs correspond to $\sigma_{r}$ and $\sigma_{-r}, r>0$.

\section{(b) Isotypic decomposition of a symplectic representation}

In this section we prove theorems 2.1 and 2.2. We begin with some further standard facts from representation theory.

Let $\mathrm{V}$ be a representation of $\mathrm{G}$. The isotypic decomposition (2.1) can be written in the form

$$
\mathrm{V} \approx \bigoplus_{j=1}^{\ell} \mathbf{k}_{j}{ }^{n_{j}} \otimes_{\mathbf{k}_{j}} \mathrm{~W}_{j}
$$

where $\mathrm{W}_{1}, \ldots, \mathrm{W}_{\ell}$ are pairwise non-isomorphic irreducible representations and

$$
\mathbf{k}_{j}=\operatorname{Hom}_{\mathbf{G}}\left(\mathbf{W}_{j}, \mathrm{~W}_{\mathrm{j}}\right) \approx \mathbb{R}, \mathbb{C} \text {, or } \mathbb{H} \text {. }
$$

Note that the action of $\mathrm{G}$ on $\mathrm{V}_{\mathrm{g}}$ is the tensor product of the action on $\mathrm{W}_{\mathrm{j}}$ and the trivial action on $\mathbf{k}_{j}{ }^{n_{j}}$. It is a consequence of Schur's lemma that any $\mathrm{N} \in \operatorname{Hom}_{\mathrm{G}}(\mathrm{V}, \mathrm{V})$ can be written as

$$
\mathrm{N}=\bigoplus_{j=1}^{\ell} \mathrm{T}_{j} \otimes_{\mathbf{k}_{j}} \mathbf{1}_{\mathrm{w}_{j}} \text {, }
$$

where $\mathbf{1}_{\mathrm{w}_{j}}$ is the identity on $\mathrm{W}_{j}$ and $\mathrm{T}_{j} \in \operatorname{Hom}_{\mathbf{k}_{j}}\left(\mathbf{k}_{j}{ }^{{ }_{j}}, \mathbf{k}_{j}{ }^{n_{j}}\right)$, the space of $\mathbf{k}_{j}$-linear transformations of $\mathbf{k}_{j}{ }^{n_{j}}$. Conversely, if $\mathrm{N}_{j} \in \operatorname{Hom}_{\mathbf{k}_{j}}\left(\mathbf{k}_{j}{ }^{n_{j}}, \mathbf{k}_{j}{ }^{n_{j}}\right)$ for $j=1, \ldots, \ell$ then (2.4) defines an element of $\mathrm{Hom}_{\mathrm{G}}(\mathrm{V}, \mathrm{V})$. Thus

$$
\operatorname{Hom}_{\mathrm{G}}(\mathrm{V}, \mathrm{V}) \approx \operatorname{Hom}_{\mathbf{k}_{\mathbf{1}}}\left(\mathbf{k}_{\mathbf{1}}{ }^{{ }_{1}}, \mathbf{k}_{\mathbf{1}}{ }^{n_{1}}\right) \oplus \ldots \oplus \operatorname{Hom}_{\mathbf{k}_{\ell}}\left(\mathbf{k}_{\ell}{ }^{{ }^{\imath}}, \mathbf{k}_{\ell}{ }^{{ }{ }^{\prime}}\right) \text {. }
$$

Restricting to invertible maps we obtain

$$
\mathbf{G L}_{\mathbf{G}}(\mathrm{V}) \approx \mathbf{G L}\left(n_{1} ; \mathbf{k}_{\mathbf{1}}\right) \times \ldots \times \mathbf{G L}\left(n_{\ell} ; \mathbf{k}_{\ell}\right) \text {. }
$$

For a uniform treatment of the cases $\mathbb{R}, \mathbb{C}, \mathbb{N}$ we must work not with symplectic forms but with closely related antihermitian forms. An antihermitian form on a k-vector space $\mathrm{X}$ is a pairing $\{\}:, X \times X \rightarrow k$ that is $\mathbf{k}$-linear in the first variable and satisfies

$$
\{y, x\}=-\{\overline{x, y}\} \text { for all } x, y \in \mathrm{X} .
$$


For $\mathbf{k}=\mathbb{R}$, conjugation is trivial, so antihermitian forms are the same as symplectic forms. For each of $\mathbf{k}=\mathbb{R}, \mathbb{C}, \mathbb{H}$ we define the standard antihermitian forms on $\mathbf{k}^{n}$ by

$$
\begin{aligned}
& \mathbf{k}=\mathbb{R}:\{x, y\}=\sum_{j=1}^{\frac{1}{3} n}\left(x_{j} y_{\frac{1}{2} n+j}-x_{\frac{1}{2} n+j} y_{j}\right) \quad(n \text { must be even }), \\
& \mathbf{k}=\mathbb{C}:\{x, y\}=\sum_{j=1}^{p} x_{j} \overline{i y_{j}}-\sum_{j=p+1}^{n} x_{j} \overline{i y_{j}} \quad(0 \leqslant p \leqslant n), \\
& \mathbf{k}=\mathbb{H}:\{x, y\}=\sum_{j=1}^{n} x_{j} \overline{i y_{j}} .
\end{aligned}
$$

Here the bar denotes complex conjugation in $\mathbb{C}$ or quaternionic conjugation in $\mathbb{H}$.

For each of $\mathbb{R}$ and $\mathbb{H}$ there is a unique standard antihermitian form, while for $\mathbb{C}$ there are $n+1$ of them. Any antihermitian form on $\mathbf{k}^{n}$ can be transformed into a standard one by a k-linear change of coordinates (see MacDonald 1979).

If $\{$,$\} is an antihermitian form then its real part$

$$
[,]=\operatorname{Re}\{,\}
$$

is a symplectic form on the underlying real vector space of $\mathbf{k}^{n}$, which satisfies

$$
[\lambda x, \lambda y]=|\lambda|^{2}[x, y] \text { for all } x, y \in \mathbf{k}^{n}, \lambda \in \mathbf{k} \text {. }
$$

Conversely any symplectic form satisfying $(2.7)$ can be used to define an antihermitian form on $\mathbf{k}^{n}$. by

$$
\begin{array}{ll}
\{x, y\}=[x, y] & \text { if } \mathbf{k}=\mathbb{R}, \\
\{x, y\}=[x, y]+[x, i y] i & \text { if } \mathbf{k}=\mathbb{C}, \\
\{x, y\}=[x, y]+[x, i y] i+[x, j y] j+[x, k y] k & \text { if } \mathbf{k}=\mathbb{H} .
\end{array}
$$

Two antihermitian forms are isomorphic by a k-linear change of coordinates if and only if the corresponding symplectic forms are similarly isomorphic. It follows that any symplectic form on $\mathbf{k}^{n}$ satisfying (2.7) can be transformed by a $\mathbf{k}$-linear change of coordinates to a standard symplectic form $[]=,\operatorname{Re}\{$,$\} , where \{$,$\} is a standard antihermitian form.$

Theorems 2.1 and 2.2 are consequences of the following result.

THEOREM 2.4. Every symplectic representation $\mathrm{V}$ of $\mathrm{G}$ has a unique decomposition

where

$$
\mathrm{V}=\mathrm{V}_{1} \oplus \ldots \oplus \mathrm{V}_{\ell},
$$

(a) The $\mathrm{V}_{1}$ are $\mathrm{G}$-invariant symplectic subspaces of $\mathrm{V}$;

(b) $\mathrm{V}_{1} \approx \mathbf{k}_{1}{ }^{n_{j}} \otimes_{\mathbf{k}_{1}} \mathrm{~W}_{1}$ where $\mathrm{W}_{1}, \ldots, \mathrm{W}_{\ell}$ are pairwise non-isomorphic irreducible representations of $\mathrm{G}$ and $\operatorname{Hom}_{\mathbb{G}}\left(\mathrm{W}_{j}, \mathrm{~W}_{j}\right) \approx \mathbf{k}_{j}=\mathbb{R}, \mathbb{C}$, or $\mathbb{H}$;

(c) The action of $\mathrm{G}$ on $\mathrm{V}_{j}$ is the tensor product of the action on $\mathrm{W}_{j}$ and the trivial action on $\mathbf{k}_{j}{ }^{{ }_{j}}$;

(d) The symplectic form on $\mathrm{V}_{1}$ can be transformed by an equivariant linear change of coordinates to one given by

$$
\left[x_{1} \otimes w_{1}, x_{2} \otimes w_{2}\right]_{\mathrm{v}_{j}}=\left[x_{1}, x_{2}\right]_{j}\left(w_{1}, w_{2}\right)_{\mathrm{w}_{j}} .
$$

Here $[$,$] , is a standard symplectic form on \mathbf{k}_{j}{ }^{{ }_{j}}$ and $(,)_{\mathrm{w}_{j}}$ is a G-invariant inner product on $\mathrm{W}_{j}$ (unique up to a scalar multiple). The symplectic forms $[$,$] , are uniquely determined by \mathrm{V}$.

Proof. Let $\mathrm{V}=\mathrm{V}_{1} \oplus \ldots \oplus \mathrm{V}$, be the usual isotypic decomposition of $\mathrm{V}$ and choose 
isomorphisms $\mathrm{V}_{j} \approx \mathbf{k}_{j}{ }^{n_{j}} \otimes_{\mathbf{k}_{j}} \mathrm{~W}_{j}$. Fix G-invariant inner products $(,)_{\mathrm{W}_{j}}$ on the $\mathrm{W}_{j}$ and let $(,)_{j}$ denote the standard inner product on $\mathbf{k}_{j}{ }^{n_{j}}$, defined by

Then

$$
(x, y)_{j}=\operatorname{Re} \sum_{k=1}^{n_{j}} x_{k} \bar{y}_{k} .
$$

defines a $\mathrm{G}$-invariant inner product on $\mathrm{V}_{j}$, and the direct sum of these gives a G-invariant inner product $(,)_{\mathbf{v}}$ on $\mathrm{V}$.

If $[,]_{\mathrm{V}}$ is the symplectic form on $\mathrm{V}$ then the formula

$$
\left[v_{1}, v_{2}\right]_{\mathrm{v}}=\left(v_{1}, \mathrm{~J}_{\mathrm{v}} v_{2}\right)_{\mathrm{v}}
$$

defines a symplectic involution $\mathrm{J}_{\mathrm{V}}: \mathrm{V} \rightarrow \mathrm{V}$. Conversely $\mathrm{J}_{\mathrm{v}}$ determines $[,]_{\mathrm{V}}$ in the presence of $(,)_{\mathrm{v}}$. A simple calculation shows that $\mathrm{J}_{\mathrm{v}}$ is G-equivariant, so by $(2.4) \mathrm{J}_{\mathrm{v}}=\oplus \mathrm{J}_{\mathrm{v}_{j}}$, where $\mathrm{J}_{\mathrm{v}_{j}}: \mathrm{V}_{j} \rightarrow \mathrm{V}_{j}$ is given by $\mathrm{J}_{\mathrm{v}_{j}}=\mathrm{J}_{j} \otimes 1_{\mathrm{w}_{j}}$ for some $\mathbf{k}_{j}$-linear map $\mathrm{J}_{j}: \mathbf{k}_{j}{ }^{n_{j}} \rightarrow \mathbf{k}_{j}{ }^{n_{j}}$.

Therefore $J_{v}$ leaves $V_{j}$ invariant, so that $V_{j}$ is a symplectic subspace of $V$. Moreover, the symplectic form restricted to $V_{j}$ is given by

$$
\begin{aligned}
{\left[x_{1} \otimes w_{1}, x_{2} \otimes w_{2}\right]_{\mathrm{v}} } & =\left(x_{1} \otimes w_{1}, \mathrm{~J}_{\mathrm{v}_{j}}\left(x_{2} \otimes w_{2}\right)\right)_{\mathrm{v}_{j}} \\
& =\left(x_{1} \otimes w_{1}, \mathrm{~J}_{j} x_{2} \otimes w_{2}\right)_{\mathrm{v}_{j}} \\
& =\left(x_{1}, \mathrm{~J}_{j} x_{2}\right)_{j}\left(w_{1}, w_{2}\right)_{\mathrm{w}_{j}} .
\end{aligned}
$$

The symplectic involution $\mathrm{J}_{j}$ gives a symplectic form $\left(x_{1}, \mathrm{~J}_{j} x_{2}\right)_{j}$ on $\mathbf{k}_{j}{ }^{n_{j}}$ which satisfies (2.7). By the discussion preceding the statement of the theorem, this is equivalent, by some $\mathbf{k}_{\mathbf{j}}$-linear change of coordinates $\Phi_{j}$, to a standard symplectic form $[$,$] , on \mathbf{k}_{j}{ }^{n_{j}}$. Define an equivariant change of coordinates $\Psi_{j}$ of $V_{j}$ by $\Psi_{j}=\Phi_{j} \otimes_{k_{j}} 1_{w_{j}}$. This transforms the restriction of $[,]_{v}$ to $\mathrm{V}_{\mathrm{j}}$ into the symplectic form $[,]_{\mathrm{v}_{\mathrm{j}}}$ defined in $(2.8)$.

The rest of the theorem merely restates standard properties of the isotypic decomposition of $\mathrm{V}$.

It is clear from this that an irreducible symplectic representation must be of the form $\mathbb{R}^{2} \otimes_{\mathbb{R}} \mathrm{W}$, where $\mathrm{W}$ is a real irreducible representation, or $\mathbf{k} \otimes_{\mathbf{k}} \mathbf{W}$, where $\mathrm{W}$ is a $\mathbf{k}$-irreducible representation, $\mathbf{k}=\mathbb{C}$ or $\mathbb{H}$. (This is the content of theorem 1.1 of Golubitsky \& Stewart (1987), which is proved there by a more concrete method.) The symplectic form is given by a standard symplectic form on $\mathbb{R}^{2}$ or $\mathbf{k}$. For the real and quaternionic cases these are unique. However, on $\mathbb{C}$ there are two non-isomorphic symplectic forms $x \bar{i} \bar{y}$ and $-x \bar{i}$, giving two non-isomorphic irreducible symplectic representations with the same underlying representation. This proves theorem 2.1. Theorem 2.2 also follows immediately.

Remark. An alternative approach to theorem 2.4 is to use a symplectic involution $\mathrm{J}_{\mathrm{V}}$ of the symplectic representation $\mathrm{V}$ to define a complex structure on $\mathrm{V}$ with respect to which the representation becomes unitary. This gives a bijection between isomorphism classes of symplectic representations and those of unitary representations. This is useful because unitary representations are widely studied. The theorem follows from the isotypic decomposition of unitary representations and the classification of irreducible unitary representations into real, complex, and quaternionic types, see Adams (1969, theorem 3.57).

Note that if $\mathrm{W}$ is a unitary representation then its dual $\mathrm{W}^{*}$ is defined by the natural action of $\mathrm{G}$ on $\mathrm{Hom}_{\mathbb{C}}(\mathrm{W}, \mathbb{C})$. If $\mathrm{W}$ is a $\mathbf{k}$-irreducible unitary representation then it is isomorphic to $\mathrm{W}^{*}$ 
if and only if $\mathbf{k}=\mathbb{R}$ or $\mathbb{R}$, although the underlying real representations are always isomorphic. This is the sense in which there are dual pairs in the complex case, and corresponds to the fact, noted above, that on $\mathbb{C}$ there are two non-isomorphic symplectic forms $x \bar{x} \bar{y}$ and $-x \bar{i} \bar{y}$.

The essential difference between the symplectic and unitary categories lies in the mappings between representations. If $\mathrm{W}$ is a complex unitary/symplectic irreducible representation then the invertible equivariant unitary linear mappings $\mathrm{W} \oplus \mathrm{W}^{*} \rightarrow \mathrm{W} \oplus \mathrm{W}^{*}$ are sums of mappings $\mathrm{W} \rightarrow \mathrm{W}$ and $\mathrm{W}^{*} \rightarrow \mathrm{W}^{*}$. They therefore form a group isomorphic to $\mathrm{U}(1 ; \mathbb{C}) \times \mathbf{U}(1, \mathbb{C})$. However, the group of equivariant symplectic linear mappings is isomorphic to $U(1,1 ; \mathbb{C})$ as we shall see in the next section.

\section{(c) Decomposition of $\mathbf{S p}_{\mathbf{G}}(\mathrm{V})$}

The groups of $\mathbf{k}$-linear transformations of $\mathbf{k}^{n}$ that preserve the standard antihermitian forms are denoted by

$$
\begin{array}{ll}
\mathbf{S p}(n ; \mathbb{R}) & \text { if } \quad \mathbf{k}=\mathbb{R}, \\
\mathbf{U}(p, q ; \mathbb{C}) & \text { if } \quad \mathbf{k}=\mathbb{C}, p+q=n, \quad \text { and } \quad\{x, y\}=\sum_{j=1}^{p} x_{j} \overline{i y_{j}}-\sum_{j=p+1}^{n} x_{j} \overline{i y_{j}}, \\
\alpha \mathbf{U}(n ; \mathbb{H}) & \text { if } \quad \mathbf{k}=\mathbb{H} .
\end{array}
$$

Here $\mathbf{S p}(n ; \mathbb{R})$ is the usual symplectic group, $\alpha \mathrm{U}(n ; \mathbb{R})$ is the quaternionic antiunitary group, and the $\alpha$ is dropped from $\mathbf{U}(p, q ; \mathbb{C})$ because any transformation preserving the antihermitian form $\{$,$\} also preserves the hermitian form i\{$,$\} , and vice versa. Also \mathbf{U}(p, q ; \mathbb{C}) \approx \mathbf{U}(q, p ; \mathbb{C})$.

If $\mathrm{V}$ is a symplectic representation of $\mathrm{G}$ then we denote the group of equivariant symplectic linear transformations of $\mathrm{V}$ by $\mathbf{S p}_{\mathrm{G}}(\mathrm{V})$. We can describe the structure of this group precisely.

TheOREM 2.5. (Structure of symplectic equivariants.) Let $\mathrm{V}=\mathrm{V}_{1} \oplus \ldots \oplus \mathrm{V}_{\ell}$, $\mathrm{V}_{j}=\mathbf{k}_{j}{ }^{n_{j}} \otimes_{\mathbf{k}_{j}} \mathrm{~W}_{j}$, be the isotypic decomposition of $\mathrm{V}$. Then

where

$$
\mathrm{Sp}_{\mathrm{G}}(\mathrm{V}) \approx \mathrm{S}_{1} \times \ldots \times \mathrm{S}_{\ell}
$$

(a) If $\mathbf{k}_{j}=\mathbb{R}$ then $\mathbf{S}_{j}=\mathbf{S p}\left(n_{j} ; \mathbb{R}\right)$;

(b) If $\mathbf{k}_{j}=\mathbb{C}$ then $\mathrm{S}_{j}=\mathbf{U}(p, q ; \mathbb{C})$, where $p$ and $q$ are determined by the standard antihermitian form on $\mathbf{k}_{j}^{n_{j}}$ defined by $\mathrm{V}$;

(c) If $\mathbf{k}_{j}=\mathbb{H}$ then $\mathrm{S}_{j}=\alpha \mathbf{U}\left(n_{j} ; \mathbb{H}\right)$.

Proof. If $N \in \mathrm{Sp}_{\mathrm{G}}(\mathrm{V})$ then by $(2.4)$

$$
\mathrm{N}=\bigoplus_{j=1}^{\ell} \mathrm{T}_{j} \otimes_{\mathrm{k}_{j}} 1_{\mathrm{w}_{j}},
$$

where $\mathbf{T}_{j}: \mathbf{k}_{j}{ }^{n_{j}} \rightarrow \mathbf{k}_{j}{ }^{n_{j}}$ is $\mathbf{k}_{j}$-linear. An easy calculation shows that if $\mathrm{N}$ is symplectic then the $\mathbf{T}_{j}$ preserve the induced symplectic form on $\mathbf{k}_{j}{ }^{{ }_{j}}$. Conversely, given $\mathbf{k}_{j}$-linear symplectic maps $\mathbf{T}_{j}$ $(j=1, \ldots, l)$ the formula $(2.4)$ defines an element of $\operatorname{Sp}_{\mathrm{G}}(\mathrm{V})$. The proof is completed by showing that a $\mathbf{k}_{j}$-linear map $\mathbf{k}_{j}{ }^{n_{j}} \rightarrow \mathbf{k}_{j}{ }^{n_{j}}$ preserves a symplectic form on $\mathbf{k}_{j}{ }^{{ }_{j}}$ satisfying (2.7) if and only if it preserves the corresponding antihermitian form. Again this is straightforward.

Example. Let $\mathrm{V}$ be the symplectic representation of $\mathrm{G}=\mathbf{S}^{\mathbf{1}}$ indexed by a sequence of integers $\left(r_{1}, \ldots, r_{k}\right)$ with $r_{1} \leqslant \ldots \leqslant r_{k}$. Let $R_{1}<\ldots<R_{\ell}$ be the values $\left|r_{j}\right|$ arranged in ascending order. Let

$$
\begin{aligned}
& n_{j}=\left|\left\{r_{i}:\left|r_{i}\right|=R_{j}\right\}\right|, \\
& p_{j}=\left|\left\{r_{i}: r_{i}=R_{j}\right\}\right|, \\
& q_{j}=\left|\left\{r_{i}: r_{i}=-R_{j}\right\}\right|=n_{j}-p_{j} .
\end{aligned}
$$


Then

$$
\begin{aligned}
& \mathbf{S p}_{\mathrm{G}}(\mathrm{V})=\mathbf{S p}\left(2 n_{1} ; \mathbb{R}\right) \times \mathbf{U}\left(p_{2}, q_{2} ; \mathbb{C}\right) \times \ldots \times \mathbf{U}\left(p_{\ell}, q_{\ell} ; \mathbb{C}\right) \text { if } R_{1}=\mathbf{0}, \\
& \mathbf{S p}_{\mathrm{G}}(\mathrm{V})=\mathbf{U}\left(p_{1}, q_{1} ; \mathbb{C}\right) \times \ldots \times \mathbf{U}\left(p_{\ell}, q_{\ell} ; \mathbb{C}\right) \text { if } R_{1}>0
\end{aligned}
$$

(d) Eigenvalues of equivariant symplectic mappings

Finally we prove theorem 2.3 . This can be done by showing that:

(i) every element of $\mathbf{U}(p, 0 ; \mathbb{C})$ and $\alpha \mathrm{U}(1 ; \mathbb{H})$ has all its eigenvalues on the unit circle, but

(ii) there exist elements of $\mathbf{S p}(2 n ; \mathbb{R})(n \geqslant 1), \mathbf{U}(p, q ; \mathbb{C})(p, q>0)$, and $\alpha \mathbf{U}(n ; \mathbb{H})(n \geqslant 2)$ with eigenvalues not on the unit circle.

There are several ways to do this. One is by direct calculation, noting that for (ii) it is sufficient to deal with $\mathbf{S p}(2 ; \mathbb{R}), \mathbf{U}(1,1 ; \mathbb{C})$ and $\alpha U(2 ; \mathbb{H})$. Another method is to exploit the Chevalley-Jordan decomposition into unipotent and semi-simple parts, see Springer (I 98I), to reduce the problem to semi-simple elements; then use the classification of semi-simple elements in Sugiura (1959). The third method is more abstract, but perhaps gets closer to the essence of the matter: it rests on the following observation.

THEOREM 2.6. $\mathrm{V}$ is cyclospectral if and only if $\mathbf{S p}_{\mathrm{G}}(\mathrm{V})$ is compact.

Proof. A compact Lie group acting on a space $\mathrm{V}$ preserves a positive definite inner product, hence can be considered as a subgroup of $\mathbf{O}(\mathrm{V})$. Therefore all eigenvalues lie on the unit circle.

Conversely, theorem 2.5 implies in particular that that $\mathbf{S p}_{\mathbf{G}}(\mathrm{V})$ is a reductive Lie group. We show that if a reductive Lie group $\mathrm{H}$ has a faithful representation $\rho$ such that all eigenvalues of $\rho(h)$ lie on the unit circle for all $h \in \mathrm{H}$, then $\mathrm{H}$ is compact. By Varadarajan (1974, p. 255 exercise 43), $\mathrm{H}=\mathrm{MA}$ where $\mathrm{M}$ and $\mathrm{A}$ are closed subgroups, $\mathrm{M}$ is semi-simple, $\mathrm{A}$ is compact abelian, and A commutes with $\mathrm{M}$. Therefore without loss of generality we may assume $\mathbf{H}$ is semi-simple. Let $\mathfrak{h}$ be the Lie algebra of $H$. Then $\rho$ induces a faithful representation of $\mathfrak{h}$, which we also denote $\rho$. Consider the trace form $\mathrm{Q}_{\rho}(\xi)=\operatorname{Tr} \rho(\xi)^{2}, \xi \in \mathfrak{h}$. This quadratic form is nondegenerate because $\mathfrak{h}$ is semi-simple, see Varadarajan (1974, lemma 3.9.5, p. 212). Because for all $h \in \mathrm{H}$ the eigenvalues of $\rho(h)$ lie on the unit circle, the eigenvalues of $\rho(\xi)$ are purely imaginary, whence the eigenvalues of $\rho(\xi)^{2}$ are real and negative. Therefore $\mathrm{Q}_{\rho}$ is negative definite (definite since it is non-degenerate). Further, $\mathrm{Q}_{\rho}$ is invariant under the adjoint representation of $\mathrm{H}$. To see this compute

$$
\operatorname{Tr}\left(\operatorname{ad}_{h} \rho(\xi)\right)^{2}=\operatorname{Tr}\left(h^{-1} \rho(\xi) h\right)^{2}=\operatorname{Tr}\left(h^{-1} \rho(\xi)^{2} h\right)=\operatorname{Tr} p(\xi)^{2} .
$$

Thus, $\operatorname{ad}(H)$ preserves a definite quadratic form. Therefore $\operatorname{ad}(H) \subset \mathbf{O}(\mathfrak{h})$ which is compact, so $\operatorname{ad}(H)$ is compact. But $\mathrm{H}$ is a covering of ad $(\mathrm{H})$, and Weyl's theorem (Varadarajan 1974, theorem 4.11.6, p. 345), implies that $\mathrm{H}$ is compact.

The proof of theorem 2.3 can now be completed as follows. By MacDonald (1979) or Tits $(1983$, p. 231) the groups $\mathbf{S p}(2 n ; \mathbb{R})$ are not compact; $\mathbf{U}(p, q ; \mathbb{C})$ is compact if and only if $p=0$ or $q=0$; and $\alpha \mathbf{U}(n ; \mathbb{H})$ is compact if and only if $n=1$.

\section{Remarks 2.7}

(a) When the representation of $\mathrm{G}$ is cyclospectral the compactness of $\mathbf{S p}_{\mathrm{G}}(\mathrm{V})$ also implies that every commuting symplectic mapping $M$ is semi-simple. That is, $M$ is linearly stable in the sense of $\S 5 b$. 
(b) Suppose that the isotypic blocks of $\mathrm{G}$ on $\mathrm{V}$ either satisfy the conditions of theorem 2.3, or have $\operatorname{Sp}_{G}\left(V_{j}\right) \approx \mathbf{S p}(2 ; \mathbb{R})$ (a symplectic irreducible real block). If $\mathrm{M} \in \mathrm{Sp}_{\mathrm{G}}(\mathrm{V})$, then those eigenvalues of $M$ that lie on the unit circle and are not equal to 1 must be semi-simple. This is true because $\mathbf{S p}(2 ; \mathbb{R})$ has the same property. Thus if $M$ is spectrally stable and has no eigenvalues equal to 1 , then it must be linearly stable.

\section{STABILITY OF EQUILIBRIUM POINTS}

In this section we apply the results of $\S 2$ to the linear stability of equilibrium points in hamiltonian systems with symmetry.

Let $\mathrm{P}$ be a symplectic manifold and $\mathrm{X}$ a hamiltonian vector field on $\mathrm{P}$ with equilibrium point $p$. Say that $p$ is spectrally stable if all eigenvalues of $\mathrm{DX}_{p}$ are imaginary, and linearly stable if in addition $\mathrm{DX}_{p}$ is semi-simple. The following result gives a representation-theoretic criterion for linear stability in equivariant systems.

Theorem 3.1. (Stability theorem for equilibria.) Let $\mathrm{P}$ be a symplectic manifold, $\mathrm{G}$ a group acting symplectically on $\mathrm{P}, \mathrm{X}$ an equivariant hamiltonian vector field and $p$ an equilibrium point of $\mathrm{X}$ with isotropy subgroup $\mathrm{H} \subset \mathrm{G}$. If the symplectic representation of $\mathbf{H}$ on $T_{p} \mathbf{P}$ is cyclospectral, then $p$ is linearly stable.

The proof rests upon the observation that $\mathrm{DX}_{p}$ is infinitesimally symplectic, i.e. it belongs to the Lie algebra $\mathfrak{s p}\left(T_{p} \mathbf{P}\right)$ of the group $\operatorname{Sp}\left(T_{p} \mathbf{P}\right)$ of all symplectic linear transformations of $T_{p} \mathrm{P}$. This is because the flow generated by $\mathrm{X}$ is symplectic. Because $\mathrm{DX}_{p}$ is equivariant with respect to the $\mathrm{H}$-action on $T_{p} \mathrm{P}$, the theorem is a corollary of the next result.

Proposition. Let $\mathrm{V}$ be a cyclospectral representation of the group $\mathrm{H}$ and let $\mathrm{L}: \mathrm{V} \rightarrow \mathrm{V}$ be an infinitesimally symplectic map that commutes with the action of $\mathrm{H}$. Then all eigenvalues of $\mathrm{L}$ are imaginary and $\mathrm{L}$ is semi-simple.

Proof. Because $\mathrm{L}$ is infinitesimally symplectic and equivariant, $\mathrm{e}^{\mathrm{L}}$ is an equivariant symplectic map, which therefore has all its eigenvalues on the unit circle in $\mathbb{C}$ and is semi-simple by remark 2.7 $a$. Therefore the eigenvalues of $\mathrm{L}$ are imaginary, and $\mathrm{L}$ is semi-simple.

\section{Remarks}

(a) Suppose that the representation of $\mathrm{H}$ on $T_{p} \mathrm{P}$ is the direct sum of a cyclospectral representation and a set of pairwise non-isomorphic real irreducible representations. Then an argument similar to that in remark $2.7 a$ shows that whenever $p$ is spectrally stable and $\mathrm{DX}_{p}$ is invertible, $p$ is also linearly stable.

(b) Liapunov stability may also be forced by a group action. We give two examples.

(i) Let $\mathrm{G}=\mathbf{S}^{\mathbf{1}}$ and $\mathbf{P}=\mathbb{C}^{k}$ with symplectic form

$$
[z, w]=\operatorname{Im} \sum_{j=1}^{k} z_{j} \bar{w}_{j}
$$

and G-action

Associated to this action is a 'momentum' $\phi$, which is conserved by any G-equivariant hamiltonian vector field $\mathrm{X}$ on $\mathbb{C}_{k}$ (see $\S 4$ ). Explicitly (see example $(a)$ in $\S 4$ )

$$
\phi(z)=\sum_{j=1}^{k} p_{j}\left|z_{j}\right|^{2}
$$


If the $p_{j}$ all have the same sign then the level sets of $\phi$ are spheres centred on the origin, which must therefore be a Liapunov stable equilibrium point of $\mathrm{X}$. However, if there exist $p_{j}$ of different sign, then it may no longer be true that the origin is a Liapunov stable equilibrium point of every equivariant hamiltonian vector field on $\mathbb{C}$, even though the representation is still cyclospectral if $p_{i} \neq-p_{j} \forall i, j$.

For example, take $k=2, p_{1}=-2$, and $p_{2}=1$, and let $\mathrm{X}$ be the vector field generated by the $\mathbf{S}^{1}$-invariant hamiltonian

$$
\mathscr{H}(z)=-2\left|z_{1}\right|^{2}+\left|z_{2}\right|^{2}+\left(z_{1} \bar{z}_{2}^{2}+\bar{z}_{1} z_{2}^{2}\right) .
$$

This has a linearly stable, but Liapunov unstable, equilibrium point at the origin, see page 5 of Holm et al. (I985), which uses a different coordinate system, and references therein.

(ii) Let $\mathrm{G}=\mathbf{S U}(2)$, realized as the unit quaternions, acting by left multiplication on $P=\mathbb{H}$. The associated momentum mapping (see example $(b)$ in $\S 4$ ) is of the form

$$
\Phi(w+x i+y j+z k)=\left(w^{2}+x^{2}-y^{2}-z^{2}, 2(x y+w z), 2(x z-w y)\right)=\left(W_{1}, W_{2}, W_{3}\right), \text { say. }
$$

Thus $\|\Phi(v)\|^{2}=W_{1}^{2}+W_{2}^{2}+W_{3}^{2}=\left(w^{2}+x^{2}+y^{2}+z^{2}\right)^{2}=\|v\|^{4}$ is conserved by the flow. But this has compact level-sets, hence the equilibrium is Liapunov stable.

(c) The stability criterion of theorem 3.1 may also be applied to relative equilibria. We recall the general situation of remark $1.2 d$. Let $\Gamma$ and $\mathrm{G}$ be compact Lie groups with commuting symplectic actions on $\mathrm{P}$. Choose $\mu \in \mathrm{g}^{*}$ and let $\mathrm{Q}=\Phi^{-1}(\mu) / \mathrm{G}_{\mu}$ be the associated reduced phase space with induced symplectic $\Gamma$-action. Let $Y$ be a $\Gamma \times G$-equivariant hamiltonian vector field on $\mathrm{Q}$. An equilibrium point $q$ of $\mathrm{Y}$ is spectrally stable if and only if the corresponding $\mathrm{G}_{\mu}$-orbit of relative equilibria in $\Phi^{-1}(\mu)$ is spectrally stable. The analogous statement is not true for linear or Liapunov stability. By theorem 3.1 the relative equilibria in $\Phi^{-1}(\mu)$ are spectrally stable if the representation of the $\Gamma$-isotropy subgroup of $q$ on $T_{q} \mathrm{Q}$ is cyclospectral. A variation on this idea is used to discuss the spectral stability of arbitrary periodic trajectories (near equilibria) in the next three sections.

\section{Decomposition of Floquet operators}

According to Noether's theorem, continuous symmetries of hamiltonian systems give rise to conserved quantities. These quantities are collected together in the momentum mapping. Conservation of momentum forces some eigenvalues of the Floquet operator $\mathbf{M}_{u}$ of a periodic trajectory to be 1. Equivariance of the hamiltonian flow forces other eigenvalues to be 1 . In this section we describe which eigenvalues are forced to be 1 . If $\Gamma$ is discrete then there are always two eigenvalues forced to 1 and this section gives no further information. We use a slight refinement of the decomposition of the tangent space to phase space introduced by Arms $e$ al. (1975), and define a quotient space on which the remaining eigenvalues lie. This quotient is precisely the tangent space to the reduced phase space of Marsden \& Weinstein (1974), when this is well defined.

In the main result of this section, theorem 4.5, we prove that the Floquet operator takes the form

$$
\mathrm{M}_{u}=\left[\begin{array}{cccc}
\mathrm{I}_{\mathrm{W}} & 0 & * & * \\
0 & \mathrm{I}_{\mathrm{X}} & 0 & 0 \\
0 & 0 & \mathrm{~N}_{u} & * \\
0 & 0 & 0 & \mathrm{I}_{\mathrm{z}}
\end{array}\right]
$$


relative to this decomposition. We also show that $\mathrm{N}_{u}$ is symplectic, and is equivariant with respect to an action of $\bar{\Sigma}_{u} \subset \Gamma \times \mathbb{R}$, where $\bar{\Sigma}_{u}$ is the inverse image of $\Sigma$ under the natural map $\Gamma \times \mathbb{R} \rightarrow \Gamma \times \mathbf{S}^{\mathbf{1}}$. In $\S 6$ we show that this action can be deformed to an action of $\Sigma$ under which $\mathrm{N}_{u}$ remains equivariant, which yields information on the eigenvalues of $\mathrm{N}_{u}$.

\section{(a) Decomposition of the tangent space}

Let $P$ be a symplectic manifold with symplectic form $\omega$ and let $G$ be a Lie group acting on $\mathbf{P}$ by symplectic diffeomorphisms. Denote the (real) Lie algebra of $\mathrm{G}$ by $\mathfrak{g}$ and its dual by $\mathfrak{g}^{*}$. The group $\mathrm{G}$ acts linearly on $\mathrm{g}$ by the adjoint action and on $\mathrm{g}^{*}$ by the coadjoint action. We assume that there exists a G-invariant momentum mapping for the action of $\mathrm{G}$ on $\mathrm{P}$. That is, an equivariant mapping $\Phi: \mathrm{P} \rightarrow \mathrm{g}^{*}$ such that for all $z \in \mathrm{P}, v \in T_{z} \mathrm{P}$, and $\xi \in \mathrm{g}$,

$$
\left\langle\mathrm{D} \Phi_{z}(v), \xi\right\rangle=\omega\left(\mathrm{X}_{\xi}(z), v\right) \text {. }
$$

Here $\mathrm{X}_{\xi}$ is the vector field on $\mathrm{P}$ generated by the 1-parameter subgroup $\exp (t \xi)$ of $\mathrm{G}$,

$$
X_{\xi}(z)=\left.(\mathrm{d} / \mathrm{d} t) \exp (t \xi) \cdot z\right|_{t=0},
$$

and $\langle$,$\rangle denotes the pairing \mathfrak{g}^{*} \times \mathfrak{g} \rightarrow \mathbb{R}$. This assumption is certainly valid locally (e.g. in the neighbourhood of a fixed point, see example $4 a$ below) if $G=\Gamma$ or $\Gamma \times \mathbb{R}$, where $\Gamma$ is a compact group and the $\mathbb{R}$-action is the flow of a $\Gamma$-invariant hamiltonian. The momentum map for $\Gamma \times \mathbb{R}$ is then the energy-momentum map for $\Gamma: \Phi_{\Gamma \times \mathbb{R}}=\left(\Phi_{\Gamma}, \mathscr{H}\right)$. Because we are concerned only with periodic trajectories near equilibria, this local existence is sufficient for our purposes.

The raison d'être of the momentum mapping is that if $\mathscr{H}$ is a G-invariant hamiltonian function on $\mathbf{P}$, then the associated flow leaves the momentum mapping invariant ("conservation of momentum'). For more details on the momentum mapping (see Marsden \& Weinstein 1974; Abraham \& Marsden 1978; Arnold 1978; Guillemin \& Sternberg 1984).

Examples. If $\mathrm{G}$ is compact then in the neighbourhood of a fixed point its action can be linearized, and if the action is symplectic then this linearization can render the symplectic form constant. For linear actions an explicit momentum mapping can be determined as follows. Let $\mathrm{V}$ be a symplectic representation of $\mathrm{G}$. This induces a representation of $\mathrm{g}$ on $\mathrm{V}$, for which $\xi \mapsto A_{\xi} \in \mathfrak{s p}(\mathrm{V})$. Then

$$
\langle\Phi(v), \xi\rangle=\frac{1}{2}\left[A_{\xi} v, v\right]
$$

is the appropriate momentum mapping. Note that $\Phi$ is a homogeneous quadratic mapping.

(a) Let $\mathrm{V}$ be a symplectic representation of $\mathbf{S}^{\mathbf{1}}$. As discussed in the examples in $\S 2$, to $\mathrm{V}$ there corresponds a sequence of integers $\left(r_{1}, \ldots, r_{k}\right)$. The momentum mapping is then

$$
\Phi(v)=\Sigma r_{j}\left|v_{j}\right|^{2},
$$

where $\mathrm{g}^{*}$ is identified with $\mathbb{R}$, and $v=\Sigma v_{j}$ is the decomposition of $v$ with respect to the irreducible representations $\sigma_{r_{j}}$.

(b) For a second example - with interesting theoretical implications - let $\mathrm{G}=\mathbf{S U}(2)$ and $\mathbf{V}=\mathbb{H}$, with symplectic form $[u, v]=\langle u, v i\rangle$. Identify $\mathbf{S U}(2)$ with the unit sphere $\mathbf{S}^{3} \subset \mathbb{H}$, acting by quaternionic left multiplication. Choosing $\{i, j, k\}$ as a basis for $\mathbf{g}$ and identifying $\mathbf{g}$ and $\mathrm{g}^{*}$ we get, for $v=w+i x+j y+z k$,

$$
\Phi(v)=\left(w^{2}+x^{2}-y^{2}-z^{2}, 2(x y+w z), 2(x z-w y)\right) .
$$

As observed in remark ( $b$ ii) of $\S 3,\|\Phi(v)\|^{2}=\|v\|^{4}$, so $\Phi$ maps spheres centred at $0 \in \mathbb{H}$ to spheres 
centred at $0 \in \mathfrak{g}^{*}$ (as it must, by equivariance). When restricted to a sphere, $\Phi$ is the Hopf fibration, and its fibres are circles. The flow of any $\mathbf{S U}(2)$-invariant hamiltonian on $\mathbb{H}$ preserves these circles, hence all its trajectories are periodic. Because the circles can all be obtained from any given circle by applying the SU(2)-action, the periods are constant on each sphere. Nonlinearities in the hamiltonian may change this period, but they leave the trajectories unchanged.

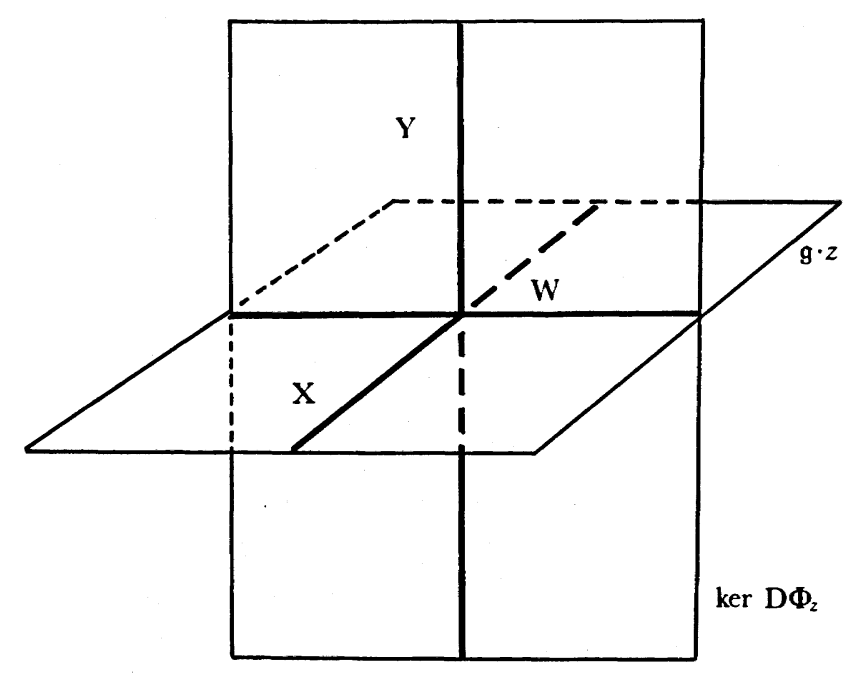

Figure 3. Decomposition of the tangent space to a symplectic manifold. The space $Z$ can be represented as a subspace perpendicular to the plane of the page.

We now take up the question of decomposing the tangent space $T_{z} \mathbf{P}$. The geometry behind the discussion that follows is shown in figure 3. Choose $z \in \mathrm{P}$ and let $\mu=\Phi(z) \in \mathrm{g}^{*}$. We define the following subspaces and quotients of $T_{z} \mathrm{P}$ :

$$
\begin{aligned}
& \mathrm{W}=\operatorname{ker} \mathrm{D} \Phi_{z} \cap \mathfrak{g} \cdot z, \\
& \mathrm{X}=\mathrm{g} \cdot z / \mathrm{W} \\
& \mathrm{Y}=\operatorname{ker} \mathrm{D} \Phi_{z} / \mathrm{W} \\
& \mathrm{Z}=T_{z} \mathrm{P} /\left(\operatorname{ker} \mathrm{D} \Phi_{z}+\mathfrak{g} \cdot z\right),
\end{aligned}
$$

where $\mathrm{g} \cdot z$ is $T_{z}(\mathrm{G} \cdot z)$, the tangent space to the group orbit through $z$. We have an isomorphism of vector spaces

$$
T_{z} \mathrm{P} \approx \mathrm{W} \oplus \mathrm{X} \oplus \mathrm{Y} \oplus \mathrm{Z}
$$

Let $\mathrm{G}_{z}$ and $\mathrm{G}_{\mu}$ denote the isotropy subgroups of the points $z$ and $\mu$ in $\mathbf{P}$ and $\mathrm{g}^{*}$ respectively, and let $\mathfrak{g}_{z}$ and $\mathfrak{g}_{\mu}$ denote their Lie algebras. Note that $G_{z} \subset G_{\mu}$ and $\mathfrak{g}_{z} \subset g_{\mu}$. There are natural actions of $\mathrm{G}_{z}$ on $T_{z} P, \operatorname{ker} \mathrm{D} \Phi_{z}$ (because $\Phi$ is equivariant), and $\mathrm{g} \cdot z$. It follows that there are induced actions of $G_{z}$ on each of $W, X, Y, Z$, though if $G$ is not compact the isomorphism (4.3) cannot in general be made $G_{z}$-equivariant. For example, the quotient $Z$ may not have a $G_{z^{-}}$ invariant representative in $T_{z} P$.

Before discussing the symplectic nature of the decomposition we need a lemma from linear algebra. For any vector space $\mathrm{V}$ we denote its dual by $\mathrm{V}^{*}$. If $\mathrm{U} \subset \mathrm{V}$ is a subspace, $\mathrm{U}^{\perp}$ will denote the annihilator of $U$ :

$$
\mathrm{U}^{\perp}=\left\{\alpha \in \mathrm{V}^{*}: \alpha \mid \mathrm{U}=0\right\}
$$


Lemma. Let $\mathrm{A}$ be a vector space and $\psi: \mathrm{A} \rightarrow \mathrm{A}^{*}$ an invertible linear map. Let $\mathrm{B} \subset \mathrm{A}$ be a subspace and denote by $\psi_{\mathrm{B}}$ the composition

$$
\mathrm{B} \longrightarrow \mathrm{A} \stackrel{\psi}{\longrightarrow} \mathrm{A}^{*} \longrightarrow \mathrm{B}^{*},
$$

the first arrou' being the natural inclusion and the third the natural projection. Let $\mathrm{C}=\psi(\mathrm{B})^{\perp} \subset \mathrm{A}$. Then

Moreover, if $\psi$ is antisymmetric, then

$$
\operatorname{ker} \psi_{\mathrm{C}}=\operatorname{coker} \psi_{\mathrm{B}}=\mathrm{B} \cap \mathrm{C} \text {. }
$$

$$
\operatorname{ker} \psi_{\mathrm{B}+\mathrm{C}}=\mathbf{B} \cap \mathbf{C} \text {. }
$$

Proof. Consider the composition

$$
\mathrm{A} \stackrel{\psi^{*}}{\longrightarrow} \mathrm{A}^{*} \longrightarrow \mathrm{B}^{*} \text {. }
$$

The kernel of this map is $\mathrm{C}$, and because $\psi_{\mathrm{B}}{ }^{*}$ is its restriction to $\mathrm{B}$, we have $\operatorname{ker} \psi_{\mathrm{B}}{ }^{*}=\mathrm{B} \cap \mathrm{C}$. Repeating the argument using

$$
\mathrm{A} \stackrel{\psi}{\longrightarrow} \mathrm{A}^{*} \longrightarrow \mathrm{C}^{*}
$$

we obtain $\operatorname{ker} \psi_{\mathrm{C}}=\mathrm{B} \cap \mathrm{C}$. The last part follows immediately.

For a subspace $\mathrm{U}$ of $T_{z} \mathrm{P}$, denote by $\omega \mid \mathrm{U}$ the restriction of $\omega$ to $\mathrm{U}$.

PROPOSITION 4.2

(a) $\operatorname{ker} \mathrm{D} \Phi_{z}$ is the $\omega$-orthogonal space to $\mathrm{g} \cdot z$.

(b) $\omega \mid\left(\mathrm{ker} \mathrm{D} \Phi_{z}+\mathrm{g} \cdot z\right)$ is singular, with null-space $\mathrm{W}$.

(c) $\omega$ induces $\mathrm{G}_{z}$-invariant symplectic forms $\omega_{\mathrm{X}}$ on $\mathrm{X}$ and $\omega_{\mathrm{Y}}$ on $\mathrm{Y}$.

(d) $\omega$ defines $a \mathrm{G}_{z}$-isomorphism of $\mathrm{W}$ with $\mathrm{Z}^{*}$. Thus the representation of $\mathrm{G}_{z}$ on $\mathrm{Z}$ is dual to that on $\mathrm{W}$.

\section{Proof}

(a) This follows immediately from (4.1).

(b) This follows from lemma 4.1, with $\psi: T_{z} \mathrm{P} \rightarrow T_{z}{ }^{*} \mathrm{P}$ given by $\psi(u)=\omega\left(u,{ }^{\bullet}\right), \mathrm{B}=\operatorname{ker} \mathrm{D} \Phi_{z}$, $\mathrm{C}=\mathrm{g} \cdot z$. The fact that $\mathrm{C}=\psi(\mathrm{B})^{\perp}$ follows from $(a)$.

(c) Similarly $\omega \mid \operatorname{ker} \mathrm{D} \Phi_{z}$ and $\omega \mid \mathrm{g} \cdot z$ both have $\mathrm{W}$ as null-space, and so induce nondegenerate symplectic forms on the quotients $\mathrm{X}$ and $\mathrm{Y}$.

(d) Because $\omega$ is $\mathrm{G}_{z}$-invariant, it follows that $\psi$ is $\mathrm{G}_{z}$-equivariant. From $(b), \psi(\mathrm{W})=$ $(\mathrm{W} \oplus \mathrm{X} \oplus \mathrm{Y})^{\perp}$, which can be naturally identified with $\mathrm{Z}^{*}$.

Remark. A symplectic form $\omega^{\prime}$ can be defined on $\mathrm{W} \oplus \mathrm{X} \oplus \mathrm{Y} \oplus \mathrm{Z}$, and representative subspaces of $T_{z} \mathrm{P}$ can be chosen, in such a way that the isomorphism (4.3) becomes symplectic. To do this define $\omega^{\prime}$ as follows:

$$
\omega^{\prime}\left|\mathrm{X}=\omega_{\mathrm{X}}, \quad \omega^{\prime}\right| \mathrm{Y}=\omega_{\mathrm{Y}}, \quad \omega^{\prime}\left|\mathrm{W}=\omega^{\prime}\right| \mathrm{Z}=0,
$$

$\omega^{\prime}(w, z)=\omega(w, z)$ for $w \in \mathrm{W}, z \in \mathrm{Z}$, which by proposition $4.2 b$ is well defined.

A particularly useful feature of the decomposition is that the actions of $G_{z}$ on three of the components, $W, X$, and $Z$, depend only on the sequence of subgroups $G_{z} \subset G_{\mu} \subset G$.

Recall that the Kostant-Kirillov-Souriau symplectic form on co-adjoint orbits (see, for example, Abraham \& Marsden 1978; Arnold 1978; Guillemin \& Sternberg 1984), is defined by a symplectic form $\Omega_{\mu}$ on $\mathfrak{g} / \mathfrak{g}_{\mu}$ via

$$
\Omega_{\mu}(\xi, \eta)=\langle\mu,[\xi, \eta]\rangle .
$$

It is easy to see that this is well defined on $\mathfrak{g} / \mathfrak{g}_{\mu}$. 


\section{Proposition 4.3}

(a) The restriction to $\mathrm{G}_{z}$ of the adjoint action of $\mathrm{G}$ on $\mathfrak{g}$ leaves invariant the subspaces $\mathfrak{g}_{\mu}$ and $\mathfrak{g}_{z}$ and so induces actions on $\mathfrak{g} / \mathfrak{g}_{\mu}$ and $\mathfrak{g}_{\mu} / \mathfrak{g}_{\mathbf{z}}$.

(b) $\left(\mathrm{X}, \omega_{\mathrm{X}}\right)$ and $\left(\mathfrak{g} / \mathfrak{g}_{\mu}, \Omega_{\mu}\right)$ are isomorphic symplectic representations of $\mathrm{G}_{\boldsymbol{z}}$.

(c) $\mathrm{W}$ and $\mathrm{g}_{\mu} / \mathrm{g}_{z}$ are isomorphic representations of $\mathrm{G}_{z}$, so $\mathrm{W} \oplus \mathrm{Z}$ is symplectically isomorphic to $\mathfrak{g}_{\mu} / \mathfrak{g}_{z} \otimes \mathbb{C}$ with standard symplectic form.

\section{Proof}

(a) The proof of this part is clear.

(b) The isomorphism is provided by the differential of the momentum mapping. Because $\Phi$ is G-equivariant, it follows that $\mathrm{D} \Phi_{z}$ is $\mathrm{G}_{z}$-equivariant. It therefore maps $\mathrm{g} \cdot z$ onto $\mathrm{g} \cdot \mu$, and $\mathrm{g} \cdot \mu$ is identified with $\mathfrak{g} / \mathfrak{g}_{\mu}$. Thus we have the exact sequence

$$
0 \longrightarrow \mathrm{W} \longrightarrow \mathrm{g} \cdot z \stackrel{\mathrm{D} \Phi_{z}}{\longrightarrow} \mathrm{g} / \mathrm{g}_{\mu} \longrightarrow 0 \text {. }
$$

So $\mathrm{g} / \mathrm{g}_{\mu}$ is $\mathrm{G}_{z}$-isomorphic to $\mathrm{g} \cdot z / \mathrm{W}$ which is by definition $\mathrm{X}$.

It remains to show that $\mathrm{D} \Phi_{z} \mid \mathrm{g} \cdot z$ preserves the restriction of the symplectic form. (It is a standard fact that $\Phi$ is 'symplectic between orbits' in this sense, but we reproduce the proof here for completeness.) Let $\xi, \eta \in \mathfrak{g}$, and let $g^{t}=\exp (t \eta)$. By the equivariance of $\Phi$,

$$
\left\langle\Phi\left(g^{t} z\right), \xi\right\rangle=\left\langle\Phi(z), \operatorname{Ad}_{g^{-t}} \xi\right\rangle \text {. }
$$

Differentiating with respect to $t$ at $t=0$, we get

$$
\left\langle\mathrm{D} \Phi_{z}\left(\mathrm{X}_{\eta}\right), \xi\right\rangle=\langle\Phi(z),[-\eta, \xi]\rangle
$$

and the right-hand side is precisely $\Omega_{\mu}(\xi, \eta)$. Furthermore, by $(4.1)$,

and we are done.

$$
\left\langle\mathrm{D} \Phi_{z}\left(\mathrm{X}_{\eta}\right), \xi\right\rangle=\omega\left(\mathrm{X}_{\xi}, \mathrm{X}_{\eta}\right)
$$

(c) Let $g \in \mathrm{G}$, and let $g: T \mathrm{P} \rightarrow T \mathrm{P}$ be the action induced from that on $\mathrm{P}$. Then

$$
g \mathrm{X}_{\xi}(z)=\mathrm{X}_{\mathrm{Ad}_{g} \xi}(g z) \text {. }
$$

If $g \in \mathrm{G}_{z}$ we get the desired result. The final statement then follows by proposition $4 d$.

Remark. If $\mu$ is a regular value of $\Phi$ and the reduced phase space $\Phi^{-1}(\mu) / \mathrm{G}_{\mu}$ is smooth at the point corresponding to $z$, then $\mathrm{Y}$ can be identified with its tangent space. See remark $1 e$.

\section{(b) Decomposition of Floquet operators}

Suppose now that $G=\Gamma \times \mathbb{R}$ where $\Gamma$ is a compact Lie group acting symplectically on $P$ and the action of $\mathbb{R}$ is given by the G-equivariant hamiltonian flow $\sigma_{t}: \mathrm{P} \rightarrow \mathrm{P}$ generated by a smooth $\Gamma$-invariant hamiltonian function $\mathscr{H}$ on P. Let $u(t)$ be a $T$-periodic trajectory of this flow with $u(0)=z$. Then the Floquet operator of $u$ is

$$
\mathrm{M}_{u}=\mathrm{D}\left(\sigma_{T}\right)_{z}: T_{z} \mathrm{P} \rightarrow T_{z} \mathrm{P} .
$$

Define $\bar{\Sigma}_{u}$ to be the isotropy group $G_{z} \subset \Gamma \times \mathbb{R}$. The new notation is chosen to suggest a relation with the symmetry group $\Sigma_{u} \subset \Gamma \times \mathbf{S}^{1}$ defined in $\S 1$. In fact, if $\nu: \Gamma \times \mathbb{R} \rightarrow \Gamma \times \mathbf{S}^{\mathbf{1}}$ is the mapping $(\gamma, t) \mapsto(\gamma,[2 \pi t / T])$, where $[\theta]$ is the equivalence class of $\theta \bmod 2 \pi \mathbb{Z}$, then $\bar{\Sigma}_{u}=\nu^{-1}\left(\Sigma_{u}\right)$.

Proposition 4.4. $\mathrm{M}_{u}$ is a $\bar{\Sigma}_{u}$-equivariant symplectic mapping.

Proof. This follows easily because $\sigma_{T}$ is symplectic and G-equivariant.

We now come to the main result of this section. 
THEOREM 4.5

(a) If we choose representative subspaces for $\mathrm{X}, \mathrm{Y}$, and $\mathrm{Z}$ in $T_{z} \mathrm{P}$, then with respect to the decomposition (4.3) $\mathrm{M}_{u}$ has the form

$$
\mathrm{M}_{u}=\left[\begin{array}{cccc}
\mathrm{I}_{\mathrm{W}} & 0 & * & * \\
0 & \mathrm{I}_{\mathrm{X}} & 0 & 0 \\
0 & 0 & \mathrm{~N}_{u} & * \\
0 & 0 & 0 & \mathrm{I}_{\mathrm{Z}}
\end{array}\right],
$$

where the $*_{s}$ represent arbitrary matrices, depending of course on the choice of representatives, and $I_{\mathrm{U}}$ is the identity map on $\mathrm{U}(\mathrm{U}=\mathrm{W}, \mathrm{X}, \mathrm{Z})$. See the remark in $\S \mathbf{6}(b)$ for further comment.

(b) The eigenvalue 1 of $\mathrm{M}_{u}$ has multiplicity at least

$$
2 \operatorname{dim} \mathrm{G} \cdot z-\operatorname{dim} \mathrm{G} \cdot \mu .
$$

(c) The map $\mathrm{N}_{u}: \mathrm{Y} \rightarrow \mathrm{Y}$ is symplectic (with respect to the symplectic form $\omega_{\mathrm{Y}}$ of proposition 4.2) and $\bar{\Sigma}_{u}$-equivariant.

Proof

(a) The map $\sigma_{T}$ is G-equivariant and so fixes the orbit $\mathrm{G} \cdot z$ pointwise, giving the form of the first two columns of $M_{u}$. The second and fourth rows of $M_{u}$ are explained by 'conservation of momentum': $\Phi \circ \sigma_{T}=\Phi$. Differentiating the equality at $z$, and taking duals we get

$$
\mathrm{M}_{u}{ }^{*} \cdot \mathrm{D} \Phi_{z}{ }^{*}=\mathrm{D} \Phi_{z}{ }^{*},
$$

so $\mathrm{M}_{u}{ }^{*}$ is the identity on the image of $\mathrm{D} \Phi_{z}{ }^{*}$. But

$$
\operatorname{Im}\left(D \Phi_{z}{ }^{*}\right)=\left(\operatorname{ker} D \Phi_{z}\right)^{\perp} .
$$

(b) Clearly the multiplicity of the eigenvalue 1 is at least

$$
\operatorname{dim} W+\operatorname{dim} X+\operatorname{dim} Z,
$$

and this is

$$
2 \operatorname{dim} \mathrm{G} \cdot z-\operatorname{dim} \mathrm{G} \cdot \mu \text {. }
$$

(c) To show that $\mathrm{N}_{u}: \mathrm{Y} \rightarrow \mathrm{Y}$ is symplectic, let $y_{1}, y_{2} \in \mathrm{Y}$ and write

for $\mathrm{E}: \mathrm{Y} \rightarrow \mathrm{W}$. Then

$$
\mathrm{M}_{u} y_{i}=\mathrm{E}_{i}+\mathrm{N}_{u} y_{i}
$$

$$
\begin{aligned}
\omega\left(y_{1}, y_{2}\right) & =\omega\left(\mathrm{M}_{u} y_{1}, \mathrm{M}_{u} y_{2}\right) \\
& =\omega\left(\mathrm{E}_{1}+\mathrm{N}_{u} y_{1}, \mathrm{E}_{2}+\mathrm{N}_{u} y_{2}\right) \\
& =\omega\left(\mathrm{N}_{u} y_{1}, \mathrm{~N}_{u} y_{2}\right),
\end{aligned}
$$

the other terms being zero by proposition $4.2 b$. The $\bar{\Sigma}_{u}$-equivariance of $\mathrm{N}_{u}$ is clear.

Remark. Even if $\Phi$ does not exist, or is not equivariant, we can still use proposition 4.2 to obtain a decomposition of $T_{z} \mathrm{P}$. On $\mathrm{g} \cdot z$ the restriction of $\omega$ has null-space denoted by $\mathrm{W}$ and $\mathrm{X}$ is defined to be $\mathrm{g} \cdot z / \mathrm{W}$. Define $\mathrm{Y}$ to be the quotient of the $\omega$-orthogonal subspace to $\mathrm{g} \cdot z$ by $\mathrm{W}$, and finally let $\mathrm{Z}$ be the quotient of $T_{z} \mathrm{P}$ by $\mathrm{W} \oplus \mathrm{X} \oplus \mathrm{Y}$. The analogue of theorem 4.4 tells us that the eigenvalue 1 of $M_{u}$ has multiplicity at least

$$
2 \operatorname{dim} \mathrm{G} \cdot z-\operatorname{rank} \omega \mid \mathrm{g} \cdot z .
$$

This is also proved in Losco (1983). 


\section{(c) Relation between the actions of $\Gamma$ and $\Gamma \times \mathbb{R}$}

To apply the theory to be developed in $\$ \S 5$ and 6 below, it is useful to investigate the relation between the symmetry properties of the momentum mappings for $\Gamma$ and for $G=\Gamma \times \mathbb{R}$ (where $\mathbb{R}$ acts via the hamiltonian flow). The aim of this section is to clarify this relation.

Let $u$ be a periodic trajectory, of fundamental period $T$, with symmetry group $\Sigma \subset \Gamma \times \mathbf{S}^{1}$. Let $z=u(0)$. Then $\mathrm{G}_{z}=\bar{\Sigma}$ is the inverse image of $\Sigma$ under the natural map $v: \Gamma \times \mathbb{R} \rightarrow \Gamma \times \mathbf{S}^{1}$, where $\nu(\gamma, t)=(\gamma,[2 \pi t / T])$ and $[\theta]$ is the equivalence class of $\theta \bmod 2 \pi \mathbb{Z}$. Let $\mathrm{H}$ be the image of $\Sigma$ under the projection $\rho: \Gamma \times \mathbf{S}^{1} \rightarrow \Gamma$, and let $\mathrm{K}=\Gamma_{z}=\Gamma \cap \Sigma$. Then $\mathrm{K} \subset \mathrm{H} \subset \Gamma$. Because $T$ is the fundamental period of $u$, the groups $H$ and $\Sigma$ are isomorphic (see Golubitsky \& Stewart $1985, \S 6)$. The twist map is the natural homomorphism $\theta: \Sigma \rightarrow \mathbf{S}^{\mathbf{1}}$ so that the elements of $\Sigma$ are of the form $(\beta(\sigma), \theta(\sigma))$, where $\beta: \Sigma \rightarrow \mathrm{H}$ is the above isomorphism. Note that Golubitsky \& Stewart (1985) define the twist as a map $\theta^{\prime}: \mathbf{H} \rightarrow \mathbf{S}^{\mathbf{1}}$ such that elements of $\Sigma$ are $\left(h, \theta^{\prime}(h)\right)$, so $\theta$ is $\beta^{-1} \circ \theta^{\prime}$.

The group $\mathrm{K}$ is equal to $\operatorname{ker} \theta$, hence is a normal subgroup of $\mathrm{H}$, and $\mathrm{H} / \mathrm{K}$ is isomorphic to a subgroup of $\mathbf{S}^{\mathbf{1}}$, which must either be the whole of $\mathbf{S}^{\mathbf{1}}$ or a finite cyclic group $\mathbf{Z}_{m}$. In the first case we say that $u$ is a rotating wave; in the second case we say it is a discrete rotating wave if $m>2$ and a standing wave if $m \leqslant 2$.

Lemma 4.6. Let $u$ be a $T$-periodic orbit with $u(0)=z$. Then $\dot{u}(0) \in T_{z}(\Gamma \cdot z)$ if and only if $u$ is a rotating wave.

Proof. If $u$ is a rotating wave then the twist is surjective, so for each $\theta \in \mathbf{S}^{1}$ there exists $\gamma_{\theta} \in \Gamma$ such that

for all $t$. Thus

$$
\gamma_{\theta} \cdot u(t+T \theta / 2 \pi)=u(t)
$$

$$
u(t)=\gamma_{2 \pi t / T}^{-1} \cdot u(0)
$$

and the trajectory lies in $\Gamma \cdot z$.

Conversely, if $\dot{u}(0)$ is tangent to $\Gamma \cdot z$ there exists $\xi$ in the Lie algebra of $\Gamma$ such that $\dot{u}(0)=$ $X_{\xi}(z)$. The curve $r(t)=\exp (t \xi) z$ is a solution curve of the hamiltonian system, because

$$
\dot{r}(t)=\exp (t \xi) \dot{u}(0)=\exp (t \xi)_{*} \mathrm{X}_{\mathrm{H}}(z)=\mathrm{X}_{\mathrm{H}}(\exp (t \xi) z)
$$

by the equivariance of the flow; so $r(t)=u(t)$ by uniqueness.

Remark. If the reduced phase space corresponding to the point $z$ is smooth, see remark $1.2 e$, then a rotating wave is a relative equilibrium.

For the remainder of this section, let $\gamma$ denote the Lie algebra of $\Gamma$. Then $\mathfrak{g}=\gamma \oplus \mathbb{R}$, and because $\mathbb{R}$ is in the centre of $G$, the adjoint or coadjoint actions of $G$ and $\Gamma$, respectively, are the same except that $\mathbb{R}$ acts trivially. That is, if $(g, t) \in \Gamma \times \mathbb{R}$ then $\operatorname{Ad}_{(g, t)}=\operatorname{Ad}_{g} \times \operatorname{Id}_{\mathbb{R}}$. Let $\Phi$ denote the momentum mapping for $\Gamma$, let $\mu=\Phi(z) \in \gamma^{*}$, and let $\mu^{\prime}=(\mu, \mathscr{H}(z)) \in \mathrm{g}^{*}$. Then $\mathrm{G} \cdot \mu^{\prime}$ and $\Gamma \cdot \mu$ can be identified, and $\mathrm{G}_{\mu^{\prime}}=\Gamma_{\mu} \times \mathbb{R}$. Because $\operatorname{dim} \Gamma \cdot z=\operatorname{dim} \mathrm{G} \cdot z$ if and only if $\dot{u}(0) \in T_{z}(\Gamma \cdot z)$, theorem 4.5 and lemma 4.6 give the following corollary.

Corollary 4.7. Let $u$ be a periodic orbit with $u(0)=z$ and $\Phi(z)=\mu$. Then the multiplicity of 1 in the spectrum of $\mathrm{M}_{u}$ is at least

if $u$ is a rotating wave, and

otherwise.

$$
\begin{gathered}
2 \operatorname{dim} \Gamma \cdot z-\operatorname{dim} \Gamma \cdot \mu \\
2 \operatorname{dim} \Gamma \cdot z-\operatorname{dim} \Gamma \cdot \mu+2
\end{gathered}
$$

Thus the lower bound for the multiplicity of 1 can be deduced from knowledge of the $\Gamma$ action and $\Sigma_{u}$. Moreover, the symplectic representations of $\mathrm{G}_{z}$ involved in the decomposition 
of $T_{z} \mathrm{P}$ in proposition 4.3 also depend only on $\Gamma$ and $\Sigma_{u}$ (or more precisely on $\Gamma, \mathrm{H}$ and $\mathrm{K}$ ), together with $\Gamma_{\mu}$. Because the coadjoint actions of $\mathrm{G}$ and $\Gamma$ differ only by the trivial action of $\mathbb{R}$, as described above, the coadjoint action of $G_{z}$ factors through the action of $H$. In fact, consider the diagram

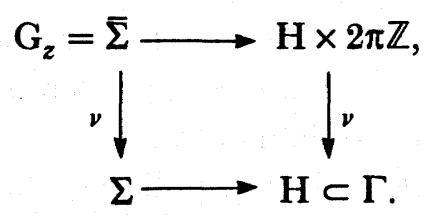

The action of $\bar{\Sigma}$ induces a natural action of $\mathrm{H}$ on $\mathrm{g}^{*}=\gamma^{*} \oplus \mathbb{R}$ by

$$
h \cdot(x, y)=(h, t(h)) \cdot(x, y),
$$

where $x \in \gamma^{*}, y \in \mathbb{R}$, and $t(h)$ is any element of $\mathbb{R}$ such that $(h, t(h)) \in \bar{\Sigma}$. Because $t(h)$ acts trivially this definition is independent of the choice of $t(h)$, and in fact

$$
(h, t(h)) \cdot(x, y)=(h \cdot x, y)
$$

because $\mathrm{H} \subset \Gamma$. Hence $h \cdot(x, y)=(h \cdot x, y)$.

More generally, suppose that groups $\mathrm{B}$ and $\mathrm{C}$ act on two spaces $\mathrm{V}$ and $\mathrm{W}$ that are isomorphic via a map $\alpha: \mathrm{V} \rightarrow \mathrm{W}$. We say that the $\mathrm{B}$-action factors through $\mathrm{C}$ if there is a group homomorphism $\beta: \mathrm{B} \rightarrow \mathrm{C}$ such that $\alpha(b \cdot v)=\beta(b) \cdot \alpha(w)$. If $\alpha$ preserves symplectic structure and $\mathrm{B}, \mathrm{C}$ act symplectically, then we say that the $\mathrm{B}$-action factors symplectically through the $\mathrm{C}$-action. Effectively we may then identify the actions of $B$ and $C$ except for a trivial part in $\operatorname{ker} \beta$. In particular, the $\mathrm{B}$-action on $\mathrm{V}$ is cyclospectral if and only if the $\mathrm{C}$-action on $\mathrm{W}$ is.

The above shows that the $\mathrm{G}_{z}$-action factors through the $\mathrm{H}$-action (provided we add the trivial space $\mathbb{R}$ to $\gamma^{*}$ for the $\mathrm{H}$-action). Routine computations lead to the following result:

PROPOSITION 4.8

(a) $\mathfrak{g} / \mathfrak{g}_{\mu^{\prime}} \approx \gamma / \gamma_{\mu}$, and the symplectic representation of $\mathrm{G}_{z}$ on $\mathrm{g} / \mathfrak{g}_{\mu^{\prime}}$ factors through that of $\mathrm{H}$ on $\gamma / \gamma_{\mu}$.

(b) $\mathfrak{g}_{\mu^{\prime}} / \mathfrak{g}_{z} \approx \gamma_{\mu} / \gamma_{z}$ if $u$ is a rotating wave, and to $\left(\gamma_{\mu} / \gamma_{z}\right) \oplus \mathbb{R}$ if not. The symplectic representation of $\mathrm{G}_{z}$ on $\mathfrak{g}_{\mu^{\prime}} / \mathfrak{g}_{z} \otimes \mathbb{C}$ factors symplectically through that of $\mathrm{H}$ on $\gamma_{\mu} / \gamma_{z} \otimes \mathbb{C}$ when $u$ is a rotating wave, and through that of $\mathbf{H}$ on $\left(\gamma_{\mu} / \gamma_{z} \oplus \mathbb{R}\right) \otimes \mathbb{C}$ when $u$ is not.

Remark. If the connected component $\Gamma^{0}$ of $\Gamma$ that contains the identity is abelian, then it acts trivially on $\gamma^{*}$, so $\Gamma_{\mu} \supset \Gamma^{0}$ for all $\mu \in \gamma^{*}$. Therefore $\gamma_{\mu}=\gamma, \gamma / \gamma_{\mu}=\{0\}$, and $\gamma_{\mu} / \gamma_{z}=\gamma / \gamma_{z}$. Thus in this case the corresponding representation of $\bar{\Sigma}=G_{z}$ can be calculated without explicit knowledge of $\Gamma_{\mu}$. In particular this is the case when $\Gamma$ is finite, because then $\Gamma^{0}=1$.

A final useful fact is the following.

Proposition 4.9. With the above notation, $\mathrm{H} \subset \Gamma_{\mu}$.

Proof. This follows directly from (4.4).

\section{STABILITY OF PERIOdig SOLUTIONS}

In the notation of $\S 1$ let $\mathscr{H}$ be a $\Gamma$-invariant hamiltonian on a symplectic manifold $\mathbf{P}$, let $p \in \mathrm{P}$ be an equilibrium, and let $u=u(t)$ be a periodic trajectory near $p$ for the $\Gamma$-equivariant hamiltonian system (1.1), obtained by applying theorem 1.1. Suppose that its period is $T_{u}$, close to $T_{p}=2 \pi /|\lambda|$, and that its isotropy subgroup is $\Sigma \subset \Gamma \times \mathbf{S}^{1}$. Let $T_{u(0)} \mathrm{P}$ be the tangent space 
to $\mathrm{P}$ at $u(0)$. If $\operatorname{dim} \mathrm{P}=2 n$ then we may identify $T_{u(0)} \mathrm{P}$ with $\mathbb{R}^{2 n}$. We recall the significance of the Floquet operator

$$
M_{u}: \mathrm{T}_{u(0)} \mathrm{P} \rightarrow \mathrm{T}_{u(0)} \mathrm{P}
$$

for the stability of $u$. For general systems a necessary condition for Liapunov stability is that all eigenvalues of $\mathbf{M}_{u}$ lie inside or on the unit circle. Because $\mathbf{M}_{u}$ is symplectic its eigenvalues form quartets $\lambda, \bar{\lambda}, \lambda^{-1}, \bar{\lambda}^{-1}$ (see Abraham \& Marsden 1978; Arnold 1978). Therefore a necessary condition for the Liapunov stability of $u$ is that all eigenvalues of $\mathrm{M}_{u}$ lie on the unit circle. If this is so we call $u$ spectrally stable. As for equilibria, $§ 3$, spectral stability is weaker than Liapunov stability. In proposition 4.4 we showed that the corresponding Floquet operator $\mathrm{M}_{u}$ is a $\bar{\Sigma}_{u}$-equivariant symplectic mapping, where $\bar{\Sigma}_{u}=\nu^{-1}(\Sigma)$ and $\nu: \Gamma \times \mathbb{R} \rightarrow \Gamma \times \mathbf{S}^{1}$ is the natural map modulo $T_{u} \mathbb{Z}$, where $T_{u}$ is the period. We wish to apply the results of $\S 3$ to $M_{u}$ to obtain a criterion for spectral stability, but $\bar{\Sigma}_{u}$ is not compact. In $\$ 5 b$ we show that in fact $\mathrm{M}_{u}$ commutes with an action of the compact group $\Sigma_{u}$, and that, when restricted to a suitable subspace, this action is isomorphic to the action of $\Sigma_{u}$ obtained by restriction from that of $\Gamma \times \mathbf{S}^{1}$ on $V_{\lambda}$. This leads to the main result of this paper, which we state as motivation below.

We begin by setting up some terminology for eigenspaces. Recall that eigenvalues of symplectic or infinitesimally symplectic mappings $\mathrm{A}$ on a space $\mathrm{V}$ come in quartets $\nu, \bar{\nu}, \nu^{-1}, \bar{\nu}^{-1}$ or $\nu, \bar{\nu},-\nu,-\bar{\nu}$, respectively. By the (real) eigenspace $\mathrm{V}_{\nu}$ corresponding to the quartet $\nu$ we mean the real part of the sum of the eigenspaces (in the complexification of $V$ ) for the members of the quartet. The same goes for generalized eigenspaces. Below we are primarily interested in the case when $\mathrm{A}$ is infinitesimally symplectic and $v$ is imaginary (so the quartet degenerates to a pair $\nu, \bar{\nu})$.

Recall that $\mathrm{L}=\mathrm{DX}_{p}$ is the linearized flow at equilibrium, and this infinitesimally symplectic. Let $\Phi: \mathrm{P} \rightarrow \mathrm{g}^{*}$ be the momentum mapping associated to the G-action as in $\S 2$. Define $\mathrm{K}=\operatorname{ker} \mathrm{D} \Phi_{u(0)}$, and let $\mathrm{E}$ be the sum of the real generalized eigenspaces of the Floquet operator $\mathrm{M}_{u}$ on $T_{u(0)} \mathrm{P}$ for eigenvalues 'near 1 ', see $\S 5 a$. Let the residual space corresponding to $\Sigma$ be

$$
\mathscr{R}_{\Sigma}=\mathrm{K} \cap \mathrm{E} / \mathrm{K} \cap \mathrm{g} \cdot u(0) .
$$

This space arises because, as we show in $\S 6$, there is a symplectic representation of the group $\Sigma$ on $\mathscr{R}_{\Sigma}$ that has strong implications for the eigenvalue structure of $\mathrm{M}_{u}$.

The main result is given in the following theorem.

Theorem 5.1. (Stability theorem.) Suppose that the hamiltonian $\mathscr{H}$ is $\Gamma$-invariant and satisfies hypotheses $\mathrm{H} 1$ and $\mathrm{H} 2$ from $\S 1$, for some $\lambda$, at the equilibrium point $p$. Let $u(t)$ be a periodic trajectory of (1.1), close to $p$, with isotropy subgroup $\Sigma \subset \Gamma \times \mathbf{S}^{1}$. Let $\mathrm{L}=\mathrm{DX}_{p}$. Assume that:

(a) all eigenvalues of $\mathrm{L}$ are on the imaginary axis;

(b). L has no eigenvalues $k \lambda / 2$ where $k \in \mathbb{Z}$ is odd;

(c) if $\mu$ is a multiple eigenvalue of $\mathrm{L}$, then $\mathrm{D}^{2} \mathscr{H}_{p}$ is definite on the (real) $\mu$ eigenspace of $L$;

(d) there are no resonances of the form $\mu_{1}-\mu_{2}=n \lambda, n \in \mathbb{Z} \backslash\{0\}$, for distinct eigenvalues $\mu_{1}$ and $\mu_{2}$ of $\mathrm{L}$ not of the form $k \lambda, k \in \mathbb{Z}$;

(e) the action of $\Sigma$ on the space $\mathscr{R}_{\Sigma}$ is cyclospectral.

Then $u(t)$ is spectrally stable for $u$ sufficiently near $p$.

\section{Remarks}

(a) Hypotheses $(a)-(d)$ of theorem 5.1 all concern data derived from the linearization $\mathrm{L}$, that is, from the quadratic part of the hamiltonian, at equilibrium. Condition $(e)$ is more subtle, but 
in many examples can also be verified from the linear data: see $\S 4 b$ and $\S 6 d$. Condition $(a)$ is obviously necessary for spectral stability of $u$. Conditions $(b),(c)$ and $(d)$ hold generically. Condition $(e)$ of course applies only to suitable isotropy subgroups $\Sigma$, but we shall show by examples in $\S \S 7$ and 8 that such isotropy subgroups occur sufficiently often for the theorem to have useful content.

(b) The idea of the theorem is that, as we describe in $\S 5 a, \mathrm{M}_{u}$ is a perturbation of $\mathrm{M}_{p}=$ $\mathrm{e}^{-2 \pi L / \lambda \mid}$, the 'Floquet operator at equilibrium for period $2 \pi /|\lambda|$ ', and thus we can infer some properties of $M_{u}$ from those of $M_{p}$. Each condition ensures that certain types of eigenvalue of $M_{u}$ are on the unit circle. If $(a)$ is not satisfied then $M_{u}$ has eigenvalues off the unit circle by a simple continuity argument. Condition (b) forbids eigenvalues of $M_{p}$, hence also of $M_{u}$, at -1 , and is related to possible period-doubling. Conditions $(c)$ and $(d)$ imply by Krein theory, see $\S 5 b$, that the eigenvalues of $M_{u}$ arising from eigenvalues of $M_{p}$ that are not close to 1 (that is, eigenspaces of $\mathrm{L}$ not contained in $\mathrm{V}_{\lambda}$ ) lie on the unit circle. Condition $(c)$ is automatically satisfied if the $\mu$-eigenspace is a symplectic irreducible, which is generically true. Condition $(d)$ can be weakened, see remark 5.10.1. Condition $(e)$ lets us apply the results of $\S 4$ to the eigenvalues of $M_{u}$ that are close to 1 . Some are forced to equal 1 by equivariance; the rest come from the space $\mathscr{R}_{\Sigma}$ and are forced to lie on the unit circle by the assumption of cyclospectrality. See $\S 5 b, c$.

\section{(a) Perturbation of the Floquet operator}

We may think of $M_{u}$ as a small perturbation of the corresponding operator $M_{p}$ for the equilibrium state. We shall describe how the Floquet operator $\mathbf{M}_{u}$ varies as we make small changes to the periodic solution $u(t)$ being studied.

We can describe the Floquet operator more concretely as follows. Identify $T_{u(0)} P$ with $\mathbb{R}^{2 n}$. and hence the equilibrium $p$ with 0 . Let $x \in T_{u(0)} \mathrm{P} \equiv \mathbb{R}^{2 n}$ and consider the non-autonomous linear system

$$
\dot{x}+\mathrm{DX}_{u(t)} x=0 .
$$

Let $x(t)$ be any solution to (5.1) and define $\mathrm{M}_{u} x(0)=x\left(T_{u}\right)$. Then $\mathrm{M}_{u}$ is the Floquet operator for $u$.

We can compare this with the situation at equilibrium. Here (5.1) becomes

$$
\dot{x}+\mathrm{L} x=0,
$$

where $\mathrm{L}=\mathrm{DX}_{p}$. This has the explicit solution $x(t)=\mathrm{e}^{-\mathrm{L} t} x(0)$, and the corresponding Floquet operator is $\mathrm{M}_{p}=\mathrm{e}^{-\mathrm{L} T_{p}}$, where $T_{p}=2 \pi /|\lambda|$. This has eigenvalues $\mathrm{e}^{-2 \pi \mu / \lambda \mid}$ as $\mu$ runs through the eigenvalues of $\mathrm{L}$.

We claim that $\mathrm{M}_{u}$ is continuous in $u$ at $u=p$. This implies that we can label the eigenvalues in such a way that each is a continuous function of $u$ at $u=p$. Essentially this is a result of classical perturbation theory, but we shall sketch a proof and make the statement more precise. To provide a suitable Banach space structure we must temporarily abandon the $C^{\infty}$ category, and in fact we prove that for arbitrary finite $k$, near $u=p$, the operator $\mathrm{M}_{u}$ varies in $\mathrm{C}^{k}$ fashion with $u$ for a suitable Banach space structure which depends on $k$.

We therefore consider a general $T$-periodic linear system

$$
\dot{x}+\mathrm{A}(t) x=0,
$$

where $\mathrm{A}(t)$ lies in a space of $\mathrm{C}^{k}$ mappings for some $k$, namely

$$
\mathscr{M}^{k}=\left\{T \text {-periodic } \mathrm{C}^{k} \text { maps } \mathbb{R} \rightarrow \mathfrak{s p}(2 n ; \mathbb{R})\right\} .
$$


A suitable norm on $\mathscr{M}^{k}$ is introduced as follows. For any linear map with matrix $C=\left(c_{i j}\right)$ define $\|C\|=\max _{i j}\left|c_{i j}\right|$, and extend this to multilinear mappings in the obvious way. For $\mathrm{A}=\mathrm{A}(t) \in \mathscr{M}^{k}$ define

$$
\|\mathrm{A}\|=\max _{1 \leqslant j \leqslant k} \sup _{t \in \mathbb{R}}\left\|\mathrm{D}^{j}{ }_{t} \mathrm{~A}(t)\right\| .
$$

Associated with each $A \in \mathscr{M}^{k}$ is its Floquet operator (or monodromy operator) $\mathbf{M}_{\mathrm{A}}$ defined by

where $x(t)$ satisfies (5.3).

$$
\mathrm{M}_{\mathrm{A}} x(0)=x(T)
$$

The following observation is no doubt well known.

Proposition 5.2. The map $\mathscr{M}^{k} \rightarrow \mathfrak{s p}(2 n ; \mathbb{R})$ associating to each $\mathrm{A} \in \mathscr{M}^{k}$ its Floquet operator $\mathrm{M}_{\mathrm{A}}$ is $C^{k}$.

Proof. We can interpret the matrix A as a parameter, taken from the Banach space $\mathscr{M}^{\boldsymbol{k}}$. The result then follows easily from smooth dependence on initial conditions and parameters of solutions of oDEs, in an appropriate infinite-dimensional setting (see Abraham et al. 1983, lemma 4.1.9, p. 190).

To deal with the Floquet equation (5.1) we must take account of the fact that the period $T_{u}$ of $u(t)$ depends on $u$. To deal with this introduce $\tau=T_{u}$ as a further parameter and rescale time by setting $t^{\prime}=\tau t / T_{p}$. Now consider the rescaled equation

$$
\mathrm{d} x / \mathrm{d} t^{\prime}+\tau / T_{p} \mathrm{DX}_{w\left(t^{\prime}\right)} x=0,
$$

where $w\left(t^{\prime}\right)=u\left(T_{p} t^{\prime} / \tau\right)$. Then we have the following.

Proposition 5.3. For each $k$, the Floquet operator $\mathbf{M}_{w}$ for (5.5) depends in a $\mathbf{C}^{k}$ fashion on $(w, \tau)$ considered as an element of $\mathscr{M}^{k} \times \mathbb{R}$.

Proof. Define $\mathrm{A}\left(t^{\prime}\right)=\tau / T_{p} \mathrm{DX}_{w\left(t^{\prime}\right)}$ and argue as before, using the parameter space $=$ $\{(w, \tau): w$ is near $\mathbf{0}$ and $\tau$ near 1$\} \subset \mathscr{M}^{k} \times \mathbb{R}$. This is an open subset of a Banach space, and the theorem is local, so the proof goes through.

\section{Remarks}

(a) In proposition 5.3 the Banach space structure depends on $k$, so the proof given here does not show that the dependence of $\mathbf{M}_{w}$ on $w$ is $C^{\infty}$. However, the referee of this paper pointed out that properties of time-ordered integrals imply that the dependence is $\mathbf{C}^{\infty}$. In this paper we require only the case $k=0$ of this proposition, and henceforth $\|u\|$ denotes the norm in $\mathscr{M}^{0}$. In that case all we need is that $T_{u}$ is continuous in $u$ at $u=p$, which is the meaning of the phrase 'period near $2 \pi /|\lambda|$ '. However, in Montaldi et al. (I988 b) we shall provide a further analysis of the dependence of the eigenvalues of $M_{u}$ on nonlinear terms in (5.1), and this will require the $C^{1}$ version of proposition 5.3.

(b) For non-hamiltonian systems a similar argument applies, but with $\mathfrak{s p}(2 n, \mathbb{R})$ replaced by $\mathfrak{g l}(2 n ; \mathbb{R})$ in the definition of $\mathscr{M}^{k}$.

Corollary 5.4. If $u(t)$ is sufficiently close to the equilibrium $x=p$, then $\mathrm{M}_{u}$ is arbitrarily close to $\mathrm{e}^{-T_{p} \mathrm{~L}}$, where $\mathrm{L}=\mathrm{DX}_{p}$.

It follows that if $u$ is near $p$ then the eigenvalues of $\mathrm{M}_{u}$ are near $\mathrm{e}^{-T_{p} \mu}$ for eigenvalues $\mu$ of L. More precisely, let $\alpha$ be an eigenvalue of $\mathrm{e}^{-T \mathrm{~L}}$ of multiplicity $m_{\alpha}$. Surround each distinct eigenvalue $\alpha$ of $\mathrm{e}^{-T_{p} \mathbf{L}}$ by a disc of radius $\epsilon$, small enough to exclude all other eigenvalues. By Rouché's theorem applied to the characteristic polynomials of $\mathrm{M}_{p}$ and $\mathrm{M}_{u}$, when $\|u\|$ is small enough, this disc still contains precisely $m_{\alpha}$ eigenvalues of $\mathbf{M}_{u}$ (counted according to multiplicity). We say that these eigenvalues of $\mathbf{M}_{u}$ emanate from $\alpha$ under perturbation of $\mathbf{M}_{u}$ from $\mathbf{M}_{p}$. 
We distinguish four types of eigenvalue of $\mathrm{M}_{p}$, as follows:

(a) eigenvalues off the unit circle;

(b) eigenvalues equal to -1 ;

(c) eigenvalues equal to 1 ;

(d) eigenvalues on the unit circle, other than \pm 1 .

We discuss these in turn.

(a) If $\mathrm{L}$ has eigenvalues off the imaginary axis, then $\mathrm{M}_{u}$ has eigenvalues off the unit circle for small enough $u$. Therefore the solution $u(t)$ will be unstable. This reduces the analysis to the case when all eigenvalues of $M_{p}$ lie on the unit circle, that is, all eigenvalues of $L$ are on the imaginary axis.

(b) Eigenvalues of $\mathrm{M}_{p}$ at -1 cause problems (related to possible period-doubling) and will be excluded by hypothesis. Generically they are absent in any case. However, if they are present, they can be removed by working with $\frac{1}{2} \lambda$ rather than $\lambda$, enlarging the space $V_{\lambda}$ to one that is technically more suitable. This process can if necessary be repeated, replacing $\lambda$ by $2^{-r} \lambda$ for suitable $r$.

(c) These correspond to the eigenvalues $k \lambda(k \in \mathbb{Z})$ of $\mathrm{L}$, because if $\mu=k \lambda$ then $\mathrm{e}^{-2 \pi \mu / \lambda \mid}=$ $\mathrm{e}^{ \pm 2 \pi 1 k}=1$. Under perturbation they break up into two types: eigenvalues that remain at 1 (due to symmetry, as in $\S 4$ ), and those 'near' 1 . We study them in detail in $\S 6$.

(d) Eigenvalues on the unit circle but not at \pm 1 will be dealt with by assuming a Krein condition, see $\S 5 b$, implying that they remain on the unit circle under perturbation. This condition is generically valid, because eigenspaces are generically $\Gamma$-simple and non-resonant.

\section{(b) Krein theory}

Recall that a symplectic transformation $\mathrm{M}: \mathrm{V} \rightarrow \mathrm{V}$ is spectrally stable if its spectrum lies on the unit circle, and it is linearly stable if in addition it is semi-simple, see $§ \$ 1$ and 3 . It is strongly stable if it lies in the interior of the set of spectrally stable symplectic transformations. We describe briefly the theory developed by Krein (1950, 1955), and also by Moser (1958), which gives necessary and sufficient conditions for $\mathrm{M}$ to be strongly stable. (See also Gelfand \& Lidskii 1955; Arnold \& Avez 1968, appendix 29; MacKay 1986).

Given $M \in \mathbf{S p}(V)$ define a quadratic form $\mathrm{Q}$ on $\mathrm{V}$ by

$$
\mathrm{Q}(x)=[\mathrm{M} x, x] .
$$

This quadratic form is clearly invariant under $M$. Let $\alpha$ be an eigenvalue of $M$. Then so are $\bar{\alpha}, \alpha^{-1}$, and $\bar{\alpha}^{-1}$. Let $E_{\alpha}$ be the real generalized eigenspace for this quartet, and let $Q_{\alpha}=Q \mid E_{\alpha}$. The eigenvalue $\alpha$ is said to be definite if $\mathrm{Q}_{\alpha}$ is definite.

Proposition 5.5. Let $\alpha$ be an eigenvalue of $M \in \mathrm{Sp}(\mathrm{V})$. Then

(a) if $\alpha \neq \pm 1$ then $\mathrm{Q}_{\alpha}$ is non-degenerate;

(b) if $|\alpha| \neq 1$ then $\mathrm{Q}_{\alpha}$ is indefinite;

(c) if $|\alpha|=1$ and $\alpha$ is simple then $\alpha$ is definite;

(d) if $\alpha$ is definite then $\mathrm{M} \mid \mathrm{E}_{\alpha}$ is semi-simple.

Proof. These results may be found, or adapted from similar results, in the cited references. Note that $(d)$ holds because $M \mid E_{\alpha}$ preserves a definite quadratic form.

Suppose now that a compact group $G$ acts symplectically on $V$, with $M \in \mathbf{S p}_{G}(V)$. As noted above, $\mathrm{Q}$ is then a G-invariant quadratic form. Define an eigenvalue $\alpha$ of $\mathrm{M}$ to be G-simple if the space $\mathrm{E}_{\alpha}$ is an irreducible symplectic subspace of $\mathrm{V}$ (in the sense of theorem 2.1). 
Proposition 5.6. Suppose that $|\alpha|=1, \alpha \neq \pm 1$, and $\alpha$ is G-simple. Then $\alpha$ is definite.

Proof. From theorem 2.1 (see also the paragraph following the proof of theorem 2.4) we consider the three types of underlying representation of $\mathrm{G}$. If the underlying representation is complex or quaternionic, then $\mathrm{E}_{\alpha}$ is itself irreducible, so the space of invariant quadratic forms is one dimensional, and all non-zero forms in it are definite. Proposition 5.5 a states that $\mathrm{Q}_{\alpha}$ is non-degenerate, hence non-zero, so it must be definite.

If the underlying representation is real, we can write $E_{\alpha}=\mathbb{R}^{2} \otimes W$, where $W$ is the underlying irreducible representation. Now $M \mid E_{\alpha}$ becomes $N \otimes 1$, where $N: \mathbb{R}^{2} \rightarrow \mathbb{R}^{2}$, and the eigenvalues of $M \mid E_{\alpha}$ are those of $N$, with multiplicity $\operatorname{dim} W$. But the eigenvalues of $N$ are on the unit circle and not real, so of the form $\alpha, \bar{\alpha}$, hence simple. If $u=x \otimes w$, then $[\mathrm{M} u, u]=$ $[\mathrm{N} x, x]_{\mathbb{R}^{2}}\langle w, w\rangle$, and $[\mathrm{N} x, x]$ and $\langle w, w\rangle$ are both definite.

TheOREM 5.7. (Krein's theorem.) Let $\mathrm{M} \in \mathrm{Sp}(\mathrm{V})$ be spectrally stable. Then $\mathrm{M}$ is strongly stable if and only if every eigenvalue is definite.

Proof. This is clear from proposition 5.5, because if every neighbourhood of M contains an $M^{\prime}$ with eigenvalues off the unit circle, then either $M$ is itself not spectrally stable, or some eigenvalue of $M$ leaves the unit circle on perturbing $M$. Because $Q_{\alpha}$ is definite, so is any nearby quadratic form, contradicting proposition 5.5.

CoROLlary 5.8. Strongly stable symplectic transformations are linearly stable.

Proof. This is an immediate consequence of Krein's theorem and proposition 5.5 d.

\section{(c) Eigenvalues far from 1}

Decompose $T_{p} P$ as $V_{\lambda} \oplus V^{\prime}{ }_{\lambda}$, where $V_{\lambda}$ is the sum of the eigenspaces of $L$ for eigenvalues that are integer multiples of $\lambda$, and $V_{\lambda}^{\prime}$ is the sum of the eigenspaces of $L$ for eigenvalues that are not integer multiples of $\lambda$. (Recall that $L$ is semi-simple.) In $\S 6$ we will discuss how $M_{p} \mid V_{\lambda}$ deforms to $M_{u} \mid E$. Here we consider how $M_{p} \mid V_{\lambda}^{\prime}$ deforms to $M_{u} \mid E^{\prime}$.

Let $\alpha$ be an eigenvalue of $M_{p} \mid V_{\lambda}^{\prime}$ with $|\alpha|=1$. Then $\alpha \neq 1$, and by hypothesis $(b)$ of the stability theorem (5.1), $\alpha \neq-1$. Let $\mathrm{U}_{\alpha} \subset \mathrm{V}_{\lambda}^{\prime}$ be the real eigenspace for the pair $\alpha, \bar{\alpha}$. Let $\mu$ be the eigenvalue of $\mathrm{L}$ with $\alpha=\mathrm{e}^{-2 \pi \mu /|\lambda|}$, which is unique by hypotheses $(c)$ and $(d)$.

Lemma 5.9. The eigenvalue $\alpha$ of $\mathrm{M}_{p}$ is definite if and only if $\mathrm{D}^{2} \mathscr{H}_{p} \mid \mathrm{U}_{\alpha}$ is definite.

Proof. By (1.1) $\mathrm{D}^{2} \mathscr{H}_{p}(x)=[L x, x]$. As in $\S 5 b$ let $\mathrm{Q}(x)=\left[\mathrm{M}_{p} x, x\right]$. Let $x \in U_{\alpha}$ and let $\xi$ be a complex eigenvector of $\mathrm{L}$ with eigenvalue $\mu$, such that $x=\xi+\bar{\xi}$. Then

and

$$
\left.\begin{array}{rl}
{[\mathrm{L} x, x]} & =(\mu-\bar{\mu})[\xi, \bar{\xi}] \\
\mathrm{Q}(x) & =(\alpha-\bar{\alpha})[\xi, \bar{\xi}] .
\end{array}\right\}
$$

The result follows.

We have now proved that the eigenvalues far from 1 remain on the unit circle, because hypotheses $(a),(c)$, and $(d)$ ensure, by the above lemma, that every eigenvalue of $M_{p} \mid V_{\lambda}^{\prime}$ is definite.

\section{Remarks $\mathbf{5 . 1 0}$}

(a) If $U_{\alpha}$ is symplectically irreducible then the invariance of $Q$ shows that $\alpha$ must be definite. It is easy to see that generically $\mathrm{U}_{\alpha}$ is symplectically irreducible.

(b) By Krein's theorem (5.7) $M_{p} \mid V_{\lambda}^{\prime}$ is strongly stable, and by continuity, so is $M_{u} \mid E^{\prime}$. By corollary $5.8, \mathrm{M}_{u} \mid \mathrm{E}^{\prime}$ is linearly stable. 
(c) For $M_{p} \mid V_{\lambda}^{\prime}$ to be strongly stable it is sufficient that each eigenvalue be definite. We can therefore weaken hypothesis $(d)$ to allow resonances of the form $\mu_{1}-\mu_{2}=n \lambda(n \in \mathbb{Z})$ provided a further 'compatibility of signs' condition is imposed on the $\mu_{1}$ and $\mu_{2}$ eigenspaces of L. Of course, we still require $\mathrm{D}^{2} \mathscr{H}_{p}$ to be definite on each L-eigenspace. From (5.6) we have

$$
(\alpha-\bar{\alpha})^{-1} \mathrm{Q}(x)=\frac{1}{2} \mu^{-1} \mathrm{D}^{2} \mathscr{H}_{p}(x)
$$

because $\mu$ is imaginary. For $\mathrm{Q}$ to be definite on $\mathrm{E}_{\alpha}$ we need the sign of $i \mu^{-1} \mathrm{D}^{2} \mathscr{H}_{p} \mid \mathrm{E}_{\alpha}$ to be constant on $\mathrm{E}_{\alpha}$. For example, if $\mu_{1}-\mu_{2}=n \lambda$ and $\mathrm{E}_{\alpha}=\mathrm{E}_{\mu_{1}} \oplus \mathrm{E}_{\mu_{2}}$, then $\mathrm{D}^{2} \mathscr{H}_{p} \mid \mathrm{E}_{\alpha}$ is definite if and only if $i \mu_{1}$ and $i \mu_{2}$ have the same sign, i.e. $\mu_{1} \mu_{2}<0$.

\section{DEFORMING THE AGTION OF $\bar{\Sigma}$}

It remains to consider the eigenvalues of $M_{u}$ that emanate from the eigenvalue 1 of $M_{p}$. We show that the action of the isotropy group $\Sigma$ of $\mathbf{M}_{u}$ imposes group-theoretic restrictions on these which, in suitable cases, imply that they remain on the unit circle. If the action of $\Sigma$ is not of such a kind, the analysis depends on the coefficients that occur in the Taylor expansion of the hamiltonian; we shall discuss this in Montaldi et al. (1988c).

We know from proposition 4.4 that $M_{u}$ is equivariant under an action of the group $\bar{\Sigma}_{u} \subset \Gamma \times \mathbb{R}$. For the remainder of this section, to simplify notation, we write $\bar{\Sigma}$ in place of $\bar{\Sigma}_{u}$, $\mathrm{M}$ in place of $\mathrm{M}_{u}$, and let $T=T_{u}$ be the period of $u$. In this section we modify the $\bar{\Sigma}$-action to produce a $\Sigma$-action (defined on the sum of the generalized eigenspaces of $M$ for the eigenvalues near 1) which retains all of its pleasant properties.

\section{(a) $\Sigma$-equivariance and eigenvalues near 1}

The obstacle to $\bar{\Sigma}$ inducing a $\Sigma$-action is that $(1, T)$ fails to act as the identity: that is, there are expanding and contracting directions for $M$. The action is therefore 'rescaled' to compensate for these contractions and expansions.

First we review some classical results on periodic linear systems, see Hale (1969, p. 119) or Hartman (1964, p. 47). We use the equation in the form

$$
\dot{x}+\mathrm{A}(t) x=0,
$$

where $x \in \mathbb{R}^{2 n}$ and $\mathrm{A}(t)=\mathrm{DX}_{u(t)}$ is a $T$-periodic infinitesinally symplectic $2 n \times 2 n$ matrix. There exists a fundamental matrix solution $\mathrm{Y}(t) \in \mathscr{M}^{0}$ such that every solution to (6.1) has the form

$$
x(t)=\mathrm{Y}(t) \cdot c
$$

for a constant vector $c \in \mathbb{R}^{2 n}$, and conversely every such $x(t)$ is a solution. Every other fundamental matrix solution is of the form $\mathrm{Y}(t) \mathrm{C}$ where $\mathrm{C}$ is a non-singular constant $2 n \times 2 n$ matrix, and anything of this form is a fundamental matrix solution. By taking $\mathrm{C}=\mathrm{Y}(0)^{-1}$ we may assume that $\mathrm{Y}(\mathbf{0})=\mathrm{I}$, which specifies $\mathrm{Y}$ uniquely.

Clearly $\mathrm{Y}(t+T)$ is also a fundamental matrix solution, so there must exist a constant matrix $\mathrm{K}$ such that

$$
\mathrm{Y}(t+T)=\mathrm{Y}(t) \mathrm{K} \text {. }
$$

Because $\mathrm{K}$ is non-singular, we can find a (possibly complex) matrix $B$ such that $K=\mathrm{e}^{T B}$. Because $\mathrm{Y}(0)=\mathrm{I}$ we have $x(t)=\mathrm{Y}(t) x(0)$. The classical trick is to define

$$
\mathrm{P}(t)=\mathrm{Y}(t) \mathrm{e}^{-\mathrm{B} t} \text {. }
$$


Then

$$
\mathrm{P}(t+T)=\mathrm{Y}(t+T) \mathrm{e}^{-\mathrm{B}(t+T)}=\mathrm{Y}(t) \mathrm{Ke}^{-\mathrm{B}(t+T)}=\mathrm{Y}(t) \mathrm{e}^{-\mathrm{B} t}=\mathrm{P}(t)
$$

so that $\mathrm{P}(t)$ is $T$-periodic. We have thus decomposed $\mathrm{Y}(t)$ as

$$
\mathrm{Y}(t)=\mathrm{P}(t) \mathrm{e}^{\mathrm{B} t},
$$

where $\mathrm{P}(t)$ is $T$-periodic and $\mathrm{B}$ is constant. Further, the Floquet operator is $\mathrm{M}=\mathrm{e}^{\mathrm{TB}}$. In particular $\mathrm{M}=\mathrm{K}$, and $\mathrm{B}$ must be some value of

$$
\mathrm{B}=1 / T \ln \mathrm{M} .
$$

Note that the entries in $\mathrm{B}$, hence in $\mathrm{P}(t)$, may as yet be complex, because the exponential map on $\mathfrak{s p}(2 n ; \mathbb{R})$ is not surjective; though it is on its complexification. Later we will need to ensure that they are real, but for the moment we ignore this point and investigate the consequences of $(6.2)$ for equivariance.

Whatever the choice of $B$ in (6.3), it must commute with $M$ because $M=e^{B T}$. We know that $M$ commutes with the action of $\bar{\Sigma}$. Indeed $M$ acts as $(1, T)$ which is in the centre of $\bar{\Sigma}$. Therefore, for all $(\gamma, \theta) \in \bar{\Sigma}$, we have

whence

$$
\begin{aligned}
\gamma \mathrm{Y}(\theta) \mathrm{M} & =\mathrm{M} \gamma \mathrm{Y}(\theta), \\
\gamma \mathrm{P}(\theta) \mathrm{e}^{\mathbf{B} \theta} \mathrm{M} & =\mathrm{M} \gamma \mathrm{P}(\theta) \mathrm{e}^{\mathbf{B} \theta}, \\
\gamma \mathrm{P}(\theta) \mathrm{Me}^{\mathbf{B} \theta} & =\mathrm{M} \gamma \mathrm{P}(\theta) \mathrm{e}^{\mathbf{B} \theta}, \\
\gamma \mathrm{P}(\theta) \mathrm{M} & =\mathrm{M} \gamma \mathrm{P}(\theta) .
\end{aligned}
$$

Thus $\mathrm{M}$ commutes with $\gamma \mathrm{P}(\theta)$. We now observe that $\gamma \mathrm{P}(\theta)$ also defines an action of $\bar{\Sigma}$, provided that a technical hypothesis holds, and that this induces an action of $\Sigma$.

Proposition 6.1. Suppose that $\mathrm{B}$ in $(6.3)$ is real and commutes with $\bar{\Sigma}$. Then there is an action * of $\bar{\Sigma}$ on $\mathbb{R}^{2 n}$ defined by $(\gamma, \theta) * x=\gamma \mathrm{P}(\theta) x$. Under * the element $(1, T)$ of $\bar{\Sigma}$ acts trivially, hence there is an induced action of $\Sigma$. If $\mathbf{B}$ is infinitesimally symplectic then the actions of $\bar{\Sigma}$ and $\Sigma$ are symplectic.

Proof. $\mathrm{e}^{\mathrm{B} \theta}$ commutes with all elements of $\bar{\Sigma}$ for any $\theta$, because $\mathrm{B}$ does. It is then easy to see that $*$ is an action (see lemma 6.5 below). Now $\mathrm{I}=\mathrm{Y}(0)=\mathrm{P}(0)$ and $\mathrm{P}$ is $T$-periodic, so $\mathrm{P}(T)=\mathrm{I}$. Thus the element $(1, T) \in \bar{\Sigma}$ acts as $1 \cdot \mathrm{P}(T)=\mathrm{I}$. Then the action of $\bar{\Sigma}$ factors through $\bar{\Sigma} / T \mathbb{Z} \approx \Sigma$. If $\mathrm{B}$ is infinitesimally symplectic then $\mathrm{e}^{\mathrm{B} \theta}$ is symplectic for all $\theta$, so the actions of $\bar{\Sigma}$ and $\Sigma$ are symplectic.

The aim now is to choose $\mathrm{B}$ in (6.3) so that

(a) B commutes with $\bar{\Sigma}$,

(b) $\mathrm{B}$ is real,

(c) B is infinitesimally symplectic, so that $\Sigma$ acts symplectically.

We also wish, when possible, to compute the symplectic isomorphism type of the action* from linear data at equilibrium. In fact there is one further technicality. The original action of $\Sigma$ is defined only on the subspace $\mathrm{V}_{\lambda}$ of $T_{p} \mathrm{P}$. Therefore we must restrict attention to a suitable subspace of $T_{u(0)} \mathrm{P}$ before performing the above construction. The idea is to treat the entire problem as a small deformation of the analogous problem at equilibrium, where everything can be calculated explicitly.

Now for the details. We have already noted that $\mathrm{M}$ can be thought of as a small perturbation of $\mathrm{M}_{p}=\mathrm{e}^{-T_{p} \mathbf{L}}$. The eigenvalues of $\mathrm{M}$ are close to $\mathrm{e}^{-\mathrm{T}_{p} \mu}$ for eigenvalues $\mu$ of $\mathrm{L}$, and the eigenvalues of interest in this subsection are those near 1 . Specifically, let $v_{1}, \ldots, v_{p}$ be the 
distinct eigenvalues of $M$ that emanate from 1 under perturbation from $M_{p}$ (where $\nu_{1}=1$ ) and define $E$ to be the (real part of the) sum of the generalized eigenspaces for the $v_{j}$. Let $E^{\prime}$ be the sum of the remaining generalized eigenspaces, so that $T_{u(0)} \mathrm{P}=\mathrm{E} \oplus \mathrm{E}^{\prime}$. The spaces $\mathrm{E}$ and $\mathrm{E}^{\prime}$ are skew-orthogonal symplectic subspaces.

Lemma 6.2

(a) $\operatorname{dim} \mathrm{E}=\operatorname{dim} \mathrm{V}_{\lambda}$.

(b) $\mathrm{E}$ is symplectic.

(c) $\mathbf{E}$ is invariant under $\overline{\boldsymbol{\Sigma}}$ and $\mathrm{M}$.

(d) $\mathrm{M} \mid \mathrm{E}$ is close to $\mathrm{I} \mid \mathrm{E}$.

(e) $\mathrm{E}$ is close to $\mathrm{V}_{\lambda}$, in the appropriate grassmannian manifold.

Proof. Parts $(a)$ and $(d)$ are obvious. Part $(b)$ follows from Williamson (1936), see also Golubitsky \& Stewart (1987, proposition 2.1) for a simple proof. To establish (c), note that by definition $E$ is invariant under $M$. However, $M$ commutes with $\bar{\Sigma}$, so $\bar{\Sigma}$ leaves all of the generalized eigenspaces of $\mathrm{M}$ invariant. Part $(e)$ is well known in perturbation theory: for example it follows from properties of Riesz projectors (see Lancaster \& Tismenetsky 1985, $\S 9.5)$.

In this section we are interested only in the eigenvalues of $\mathrm{M}$ on $\mathrm{E}$. The action of $\bar{\Sigma}$ leaves $\mathrm{E}$ invariant, and on it takes the form

$$
(\gamma, \theta) x(0)=\gamma x(\theta) .
$$

On $\mathrm{E}$ there is a natural choice for the matrix $\mathrm{B}$ in (6.3), as we now show. We define a matrix $\mathbf{B}^{\prime}$ by the logarithmic series:

$$
\mathrm{B}^{\prime}=1 / T \ln \mathrm{M}=1 / T \sum_{j=1}^{\infty}(1 / j)(-1)^{j+1}((\mathrm{M}-\mathrm{I}) \mid \mathrm{E})^{j} .
$$

This series converges because $\mathrm{M} \mid \mathrm{E}$ is close to $\mathrm{I} \mid \mathrm{E}$. The entries of $\mathbf{B}^{\prime}$ are obviously real.

Lemma 6.3

(a) $\mathbf{B}^{\prime}$ is infinitesimally symplectic on $\mathbf{E}$.

(b) $\mathbf{B}^{\prime}$ commutes with $\overline{\mathbf{\Sigma}}$ on $\mathbf{E}$.

(c) $\mathrm{B}^{\prime}$ commutes with $\mathrm{M}$ on $\mathbf{E}$.

(d) $\mathbf{B}^{\prime}$ is close to $0 \mid \mathbf{E}$.

(e) If $\mathbf{B}$ in (6.3) is chosen so that $\mathbf{B} \mid \mathbf{E}=\mathbf{B}^{\prime}$, then the $\bar{\Sigma}$-action on $\mathrm{E}$ is given by

Proof

$$
(\gamma, \theta) \cdot x=\gamma \mathrm{P}(\theta) \mathrm{e}^{\mathrm{B}^{\prime} \theta} x
$$

(a) For simplicity drop the $\mid \mathrm{Es}$. We have $\mathrm{M} \in \mathbf{S p}(\mathrm{E})$. The exponential map

$$
\exp : \mathfrak{s p}(E) \rightarrow \mathbf{S p}(E)
$$

is bijective near 0, see Adams (1969), and $\exp \left(T B^{\prime}\right)=M \mid E \in S p(E)$, whence $T B^{\prime} \in \mathfrak{s p}(E)$ so $\mathbf{B}^{\prime} \in \mathfrak{s p}(\mathrm{E})$.

$(b, c, d)$ These are clear from the series $(6.5)$.

(e) This follows because $\mathrm{e}^{\mathrm{B}^{\prime} T}=\mathrm{M}$ on $\mathrm{E}$.

Because $\mathbf{E}$ is invariant under $M$ we can indeed choose $B$ so that $B \mid E=B^{\prime}$, and this we now do. We next 'scale away' the $\mathbf{B}^{\prime}$ part of the $\bar{\Sigma}$-action, when restricted to E, leaving only the $\mathbf{P}(t)$ part, which is $T$-periodic (and will therefore induce a $\Sigma$-action). To be precise, we shall prove a variation on proposition 6.1 : 
Theorem 6.4. For u sufficiently near $p$ there is a symplectic action $*$ of $\bar{\Sigma}$ on $T_{u(0)} \mathbf{P}$ which restricts to the action of $\Sigma$ on $\mathrm{E}$ defined by

$$
(\gamma, \theta) * x=\gamma \mathrm{P}(\theta) x,
$$

and $\mathrm{M} \mid \mathrm{E}$ commutes with this action. The action (6.7) is symplectically isomorphic to the one obtained by restriction from the action of $\Gamma \times \mathbf{S}^{1}$ on $\mathrm{V}_{\lambda}$.

Remark. We emphasize that this is a $\Sigma$-action, not just a $\bar{\Sigma}$-action.

Theorem 6.4 follows from a simple algebraic fact: two commuting actions of a group can be combined by performing them in turn. This is proved by the following trivial calculation.

Lemma 6.5. Let $\bullet$ and $\diamond$ be two actions of a group $\mathrm{G}$ on a space $\mathrm{Z}$, which commute in the sense that

$$
g \diamond(h \bullet z)=h \bullet(g \diamond z)
$$

for all $g, h \in \mathrm{G}$ and $z \in \mathrm{Z}$. Then the map $\square: \mathrm{G} \times \mathrm{Z} \rightarrow \mathrm{Z}$ defined by

$$
g \square z=g \bullet(g \diamond z)
$$

is a G-action. If $\bullet$ and $\diamond$ are symplectic then so is $\square$.

Proof of theorem 6.4. Define $\mathrm{B}^{\prime \prime}: T_{u(0)} \mathrm{P} \rightarrow T_{u(0)} \mathrm{P}$ as follows. Choose $\mathrm{B}$ in (6.4) so that $\mathrm{B} \mid \mathrm{E}=\mathrm{B}^{\prime}$. Let $B^{\prime \prime} \mid E=B^{\prime}$, and let $B^{\prime \prime}$ be zero on $E^{\prime}$, the natural skew-orthogonal complement to $E$ on $T_{u(0)} \mathrm{P}$. Apply lemma 6.5 with $\mathrm{G}=\bar{\Sigma}, \mathrm{Z}=\mathrm{E}$, • the action

and $\diamond$ defined by

$$
\begin{gathered}
(\gamma, \theta) \bullet x(0)=\gamma x(\theta)=\gamma \mathrm{P}(\theta) \mathrm{e}^{\mathrm{B} \theta} x(0), \\
(\gamma, \theta) \diamond x(0)=\mathrm{e}^{-\mathrm{B}^{*} \theta} x(0) .
\end{gathered}
$$

Clearly $\diamond$ is also a symplectic action; and (6.8) follows from lemma 6.2 $a$. Then the action is the same as * in $(6.7)$. Now * is a $\bar{\Sigma}$-action; but $(1, T)$ acts on $\mathrm{E}$ by $\mathrm{P}(T) \mathrm{e}^{T \mathrm{~B}} \mathrm{e}^{-T \mathrm{~B}^{\prime}}=\mathrm{I}$, because $\mathrm{P}(T)=\mathrm{P}(0)=\mathrm{I}$ and $\mathrm{B}\left|\mathrm{E}=\mathrm{B}^{\prime}\right| \mathrm{E}$. We may therefore define a $\Sigma$-action on $\mathrm{E}$ by factoring out the subgroup $T \mathbb{Z}$ which acts trivially via *. Now on $E$ the Floquet operator $M$ commutes with $\bar{\Sigma}$ and with B, so it commutes with the action of $\Sigma$.

Finally we prove that the action (6.7) of $\Sigma$ is symplectically isomorphic to its action on $V_{\lambda}$, where

$$
(\gamma, \theta) \cdot x_{0}=\gamma \mathrm{e}^{-\theta \mathrm{L}} x_{0} .
$$

We do this by a deformation argument, based on computing the action when $u(t) \equiv p$ and showing that because it changes continuously it must retain its symplectic isomorphism type.

First consider what the action $*$ is for the equilibrium solution $x=p$ considered as a $T$ periodic solution $u(t) \equiv p$. On $\mathrm{V}_{\lambda}$ the Floquet operator $\mathrm{M}_{p}$ is $\mathrm{e}^{-T_{p} \mathrm{~L}}$, which is the identity. So $\mathrm{B}=0$ and $\mathrm{P}(t)=\mathrm{e}^{-\mathrm{L} t}$. Thus the $*$-action of $\Sigma$ on $\mathrm{V}_{\lambda}$ is given by $(6.10)$ when $u(t) \equiv 0$.

Now deform $u$ continuously away from $p$. The entire construction varies continuously, and in particular $\mathrm{V}_{\lambda}$ changes continuously into $\mathrm{E}$, see lemma $6.2 e$. Hence the action $(6.10)$ deforms continuously into the $*$-action of $\boldsymbol{\Sigma}$ on $\mathrm{E}$. But it is a standard fact about symplectic representations of compact Lie groups that they retain their isomorphism type under continuous deformation. This is a simple consequence of the orthogonality of characters. Recall that to each symplectic representation there is associated a unique unitary representation $(\S 2)$. The character of a unitary representation $\rho$ is the function $\chi: \Gamma \rightarrow \mathbb{C}$ given by $\chi(\gamma)=\operatorname{tr} \rho(\gamma)$. 
Isomorphic representations have the same character. There is an inner product on characters defined by

$$
\left\langle\chi_{1}, \chi_{2}\right\rangle=\int_{\Gamma} \chi_{1}(\gamma) \bar{\chi}_{2}(\gamma) .
$$

If $\chi$ and $\chi^{\prime}$ are irreducible characters then $\left\langle\chi, \chi^{\prime}\right\rangle=0$ unless the corresponding representations are isomorphic. If they are isomorphic then $\chi^{\prime}=\chi$ and $\langle\chi, \chi\rangle=1$. The multiplicity with which a representation having $\chi$ as its character appears in the decomposition of a representation having character $\xi$ is

$$
\langle\chi, \xi\rangle \text {. }
$$

As the representation deforms continuously, so does (6.11). But, being an integer, it must remain constant; hence the unitary isomorphism type of the representation, i.e. the symplectic isomorphism type of the original representation, cannot change.

\section{Remarks}

(a) Although we have made $\mathrm{M}$ commute with $\Sigma$ on $\mathrm{E}$ it seems unlikely that in general we can arrange this (for a non-trivial action of $\Sigma$ ) on all of $T_{u(0)} \mathrm{P}$, because the exponential function is not surjective. Under extra hypotheses, however, we can adapt the approach above to a more global setting. We shall not pursue the question here.

(b) By deleting all references to the symplectic structure, we can obtain a similar result in the analogous context of periodic solutions arising by Hopf bifurcation, as in Golubitsky \& Stewart (1985).

\section{(b) Proof of the stability theorem}

To complete the proof of the stability theorem 5.1 we apply the above construction in the context of $\S 4$. We set $z=u(0)$, so that $T_{u(0)} \mathrm{P}=T_{z} \mathrm{P}$, and write $\mathrm{K}=\operatorname{ker} \mathrm{D} \Phi_{z}$. We have a lattice of subspaces

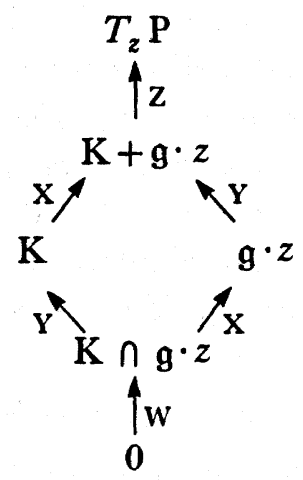

Here the annotations on the arrows denote the (isomorphism type of the) corresponding quotient spaces, introduced in $\S 4$. The eigenvalues of $\mathrm{M}$ on $\mathrm{W}, \mathrm{X}, \mathrm{Z}$ are all 1 .

There are two actions of $\bar{\Sigma}$ on $T_{z} \mathrm{P}$ : the original action $(\cdot)$ inherited from $\Gamma \times \mathbb{R}$, and the modified action $(*)$ of $(6.7)$. The latter induces an action of $\Sigma$ on $\mathrm{E} \subset T_{z} \mathrm{P}$. All subspaces in (6.12) are invariant under the original $\bar{\Sigma}$-action, and hence also under $M=(1, T) \in \bar{\Sigma}$.

Lemma 6.6. For $\|u\|$ sufficiently small, if a subspace of $T_{z} \mathrm{P}$ is invariant under the original action of $\bar{\Sigma}$, then it is invariant under the modified action.

Proof. Let $U \subset T_{z} \mathrm{P}$ be $\bar{\Sigma}$-invariant. Then $\mathrm{U}=\mathrm{U}_{1} \oplus \mathrm{U}_{2}$ where $\mathrm{U}_{1}$ is the sum of the 
generalized eigenspaces of $M \mid U$ for eigenvalues near 1 , and $U_{2}$ is the sum of the remaining generalized eigenspaces. We have $\mathrm{U}_{1}=\mathrm{U} \cap \mathrm{E}, \mathrm{U}_{2}=\mathrm{U} \cap \mathrm{E}^{\prime}$. On $\mathrm{U}_{2}$ the modified action is the same as the original, so for all $(\gamma, \theta) \in \bar{\Sigma}$ we have $(\gamma, \theta) * \mathrm{U}_{2} \subset \mathrm{U}_{2}$. Now $\mathrm{M}$ leaves $\mathrm{U}_{1}$ invariant, so $\mathrm{e}^{-\mathrm{B}^{\prime} \theta}$ leaves $\mathrm{U}_{1}$ invariant. If $x \in \mathrm{U}_{1}$ then $(\gamma, \theta) * x=(\gamma, \theta) \cdot \mathrm{e}^{-\mathrm{B}^{\prime} \theta} x \in \mathrm{U}_{1}$.

Thus when we say that a subspace of $T_{z} \mathrm{P}$ is $\bar{\Sigma}$ - or M-invariant, we need not specify which action is intended, provided we establish invariance for the original action.

We now prove the following.

Proposition 6.7. If the action of $\Sigma$ on $\mathrm{K} \cap \mathrm{E} / \mathrm{K} \cap \mathrm{g} \cdot z$ is cyclospectral, then $\mathrm{M} \mid \mathrm{E}$ is spectrally stable.

Proof. Note that $\mathrm{K} \cap \mathfrak{g} \cdot z \subset \mathrm{E}$ by the definition of $\mathrm{E}$. Consider the sequence

$$
\mathrm{E} \supset \mathrm{K} \cap \mathrm{E} \supset \mathrm{K} \cap \mathfrak{g} \cdot z \supset 0 .
$$

These spaces are $M$-invariant and the eigenvalues of $M$ on $E / K \cap E$ and on $K \cap g \cdot z$ are 1 by theorem 4.5. The space $\mathrm{K} \cap \mathrm{E} / \mathrm{K} \cap \mathfrak{g} \cdot z$ is symplectic by proposition $4.2 c$, and $\bar{\Sigma}$ acts symplectically on it, hence so does $\Sigma$. The result follows from the definition of cyclospectrality.

This completes the proof of theorem 5.1.

Remark. Unlike the case of equilibria, we cannot assert here that $\mathbf{M}_{u}$ is linearly stable when $\Sigma$ is cyclospectral on $\mathscr{R}_{\Sigma}$. This is because of the presence of off-diagonal blocks, marked *, in the matrix of theorem $4.5 a$.

If the eigenvalues of $M_{u}$ on the space $Y$ of theorem 4.5 are not 1 , then we can choose $Y$ to be the sum of the corresponding generalized eigenspaces, which is $\mathbf{M}_{u}$-invariant. This implies that the only possible off-diagonal block is the $(\mathrm{W}, \mathrm{Z})$, entry. This part of the matrix can be interpreted as a linear drift along the constant-momentum part of the orbit of periodic trajectories, and there is no reason to expect to be able to rule out such a drift. Indeed the 'correct' definition of linear stability of a periodic trajectory, when a symmetry group is present, should permit such a non-zero block in the $(\mathrm{W}, \mathrm{Z})$ position.

We call $\mathscr{R}_{\Sigma}=\mathrm{K} \cap \mathrm{E} / \mathrm{K} \cap \mathrm{g} \cdot z$ the residual near-1 eigenspace, or more briefly the residual space, for $\Sigma$. We emphasize $\Sigma$ in the notation because it is the residual representation of $\Sigma$, that is the representation of $\Sigma$ on $\mathscr{R}_{\Sigma}$, that is crucial. Similarly we call $\mathrm{M}_{u} \mid \mathscr{R}_{\Sigma}$ the residual Floquet operator. When $\mathrm{V}=\mathrm{V}_{\lambda}$, the space $\mathscr{R}_{\Sigma}$ is the same as $\mathrm{Y}$ in $\S 4$. We now investigate how to compute the residual representation.

\section{(c) Computing the action of $\Sigma$}

To apply the stability theorem to specific examples we must be able to compute the action of $\Sigma$ on $\mathscr{R}_{\Sigma}=\mathrm{K} \cap \mathrm{E} / \mathrm{K} \cap \mathrm{g} \cdot z$. In this subsection we derive a formula for the character of this representation, which depends on linear data at the equilibrium point, together with one further piece of information: the momentum isotropy subgroup $\Gamma_{\mu}$ where $\mu=\Phi(u(0))$ and $\Phi$ is the momentum mapping for $\Gamma \times \mathbb{R}$. From this definition $\Gamma_{\mu}$ apparently depends on the $\mathbb{R}$ action, that is, the hamiltonian flow. However, in the next subsection we show that in suitable cases $\Gamma_{\mu}$ can be found without knowing the details of this flow.

We know from the remark after theorem 2.4 that to each symplectic representation of $\Sigma$ on a symplectic space $U$ there corresponds a unique unitary representation on $U$ (with complex structure induced by a symplectic involution). We let $\chi[\mathrm{U}]$ denote the (complex) character of this representation. Then we have the following. 
Proposition 6.8

$$
\chi\left[\mathscr{R}_{\Sigma}\right]=\chi\left[\mathrm{V}_{\lambda}\right]-\chi\left[\mathfrak{g}_{\mu} / \mathfrak{g}_{z} \otimes \mathbb{C}\right]-\chi\left[\mathfrak{g} / \mathfrak{g}_{\mu}\right] .
$$

Remark. Before giving the proof we explain the notation. The character $\chi\left[\mathrm{V}_{\lambda}\right]$ is that of the representation of $\Sigma$ on $V_{\lambda}$ obtained by restriction from that of $\Gamma \times S^{1}$. By proposition $4.3 b \mathrm{~g} / \mathrm{g}_{\mu}$ is symplectically isomorphic to $\mathrm{g} \cdot z / \mathrm{K} \cap \mathrm{g} \cdot z$ as a $\bar{\Sigma}$-space. The coadjoint action of $\mathbb{R} \subset \Gamma \times \mathbb{R}$ on $\mathfrak{g}$ is trivial, hence the $\bar{\Sigma}$-action induces a $\Sigma$-action. Its unitary character is $\chi\left[\mathbf{g} / \mathfrak{g}_{\mu}\right]$. Similarly there is a symplectic isomorphism between

$$
(\mathrm{K} \cap \mathrm{g} \cdot z) \oplus\left(T_{z} \mathrm{P} /(\mathrm{K}+\mathrm{g} \cdot z)\right)^{*}
$$

and

$$
\mathfrak{g}_{\mu} / \mathfrak{g}_{z} \otimes \mathbb{C}
$$

as $\bar{\Sigma}$-spaces; and again $\mathbb{R}$ acts trivially on the latter, whence there is a $\Sigma$-action whose character we denote $\chi\left[\mathfrak{g}_{\mu} / \mathfrak{g}_{z} \otimes \mathbb{C}\right]$. All characters on the right-hand side of $(6.13)$ are in principle computable, hence so is the character on the left-hand side, which is what we require. In practice we compute the second two terms on the right-hand side by using proposition 4.8 , and in some cases the subsequent remark.

Proof. For the original action of $\bar{\Sigma}$ we have

$$
\chi[\mathrm{E}]=\chi[\mathrm{E} / \mathrm{K} \cap \mathrm{E}]+\chi\left[\mathscr{R}_{\Sigma}\right]+\chi[\mathrm{K} \cap \mathfrak{g} \cdot z] .
$$

Now $\mathrm{E} / \mathrm{K} \cap \mathrm{E} \approx \mathrm{E}+\mathrm{K} / \mathrm{K}$. We claim that $\mathrm{E}+\mathrm{K}=T_{z} \mathrm{P}$. This is clear because the eigenvalues of $\mathrm{M}$ on $T_{z} \mathrm{P} / \mathrm{K}$ are all 1 . Because $\mathrm{K}$ is $\mathrm{M}$-invariant the sum of the generalized eigenspaces of $\mathrm{M}$ on $T_{z} \mathrm{P} / \mathrm{K}$ for eigenvalues near 1 must be $\mathrm{E}+\mathrm{K} / \mathrm{K}$. Therefore $\mathrm{E}+\mathrm{K}=T_{z} \mathrm{P}$. So for the modified action (*) of $\bar{\Sigma}$ we also have (6.14), which we rewrite as

$$
\chi\left[\mathscr{R}_{\Sigma}\right]=\chi[\mathrm{E}]-\chi[\mathrm{E} / \mathrm{K} \cap \mathrm{E}]-\chi[\mathrm{K} \cap \mathrm{g} \cdot z] .
$$

On $\mathrm{E} / \mathrm{K} \cap \mathrm{E}$ and $\mathrm{K} \cap \mathfrak{g} \cdot z$ we have $\mathrm{M}=\mathrm{I}$, so the original and modified actions of $\bar{\Sigma}$ coincide; further we can interpret the modified $\bar{\Sigma}$-action as a $\Sigma$-action. Hence

$$
\begin{aligned}
\chi[\mathrm{E} / \mathrm{K} \cap \mathrm{E}]+\chi[\mathrm{K} \cap \mathfrak{g} \cdot z] & =\chi\left[T_{z} \mathrm{P} / \mathrm{K}\right]+\chi[\mathrm{K} \cap \mathfrak{g} \cdot z] \\
& =\chi\left[\mathfrak{g}_{\mu} / \mathfrak{g}_{z} \otimes \mathbb{C}\right]+\chi\left[\mathfrak{g} / \mathfrak{g}_{\mu}\right] .
\end{aligned}
$$

Finally $\mathrm{E}$ is $\Sigma$-isomorphic to $\mathrm{V}_{\lambda}$, so $\chi[\mathrm{E}]=\chi\left[\mathrm{V}_{\lambda}\right]$. The result follows.

We will give examples showing how to use proposition 6.8 in $\S \S 7$ and 8.

$$
\text { (d) Calculation of } \Gamma_{\mu}
$$

By the results of $\S 4 b$ everything in (6.13) can be computed from linear data at equilibrium, provided that we also know $\Gamma_{\mu}$. This appears to be a more subtle question and we are unable to give a general answer. However, we can obtain upper and lower 'bounds' for $\Gamma_{\mu}$ which turn out to be sufficient for the calculations in the next two sections.

The lower bound has already been described in $\S 4$. If $H$ is the projection of $\Sigma$ (or equivalently of $\overline{\boldsymbol{\Sigma}}$ ) into $\Gamma$, then $\Gamma_{\mu} \supset \mathrm{H}$. The upper bound will follow from the next result. As usual P is a symplectic manifold with a $\Gamma$-action which fixes $p$, and $\Phi$ is the momentum mapping on $P$. The tangent space $T_{p} \mathrm{P}$ is denoted by $\mathrm{V}$. The group $\Gamma$ acts on $\mathrm{V}$, hence there is also a momentum mapping $\Psi: \mathrm{V} \rightarrow \gamma^{*}$ where $\gamma$ is the Lie algebra of $\Gamma$.

Proposition 6.9. Let $\mathrm{S}$ be a submanifold of $\mathrm{P}$ containing $p$. Then there exists a neighbourhood $\mathrm{U}$ of 
$p$ in $\mathrm{S}$ with the following property: if $z \in \mathrm{U} \backslash\{p\}$ then there exists $w \in T_{p} \mathrm{~S} \backslash\{0\}$ such that $\Gamma_{\mu}$ is conjugate to a subgroup of $\Gamma_{\eta}$, where $\mu=\Phi(z)$ and $\eta=\Psi(w)$.

Proof. Because the $\Gamma$-action may be linearized near $p$ we may assume that $\mathrm{P}=\mathrm{V}, p=0$. Any two momentum maps for the same action differ by a constant, so $\Phi-\Psi=\tau \in \gamma^{*}$. The equivariance of $\Phi$ and $\Psi$ implies that $\tau$ is fixed by the coadjoint action, so without affecting any isotropy subgroups we can suppose that $\tau=0$ and thus $\Phi=\Psi$. From the remarks on examples in $\S 4 a$ we know that $\Psi$ is a homogeneous quadratic mapping. Assume for a contradiction that the proposition is false. Then there exists a sequence of points $\left\{z_{i}\right\}$ in $\mathrm{S}$ converging to $p$ and such that, if $\mu_{i}=\Psi\left(z_{i}\right)$, then $\Gamma_{\mu_{i}}$ is not conjugate to a subgroup of $\Gamma_{\eta}$ for any $\eta \in \gamma^{*}$ such that $\eta=\Psi(w)$ for some $w \in T_{0} S \backslash\{0\}$. Because $\Psi$ is homogeneous,

$$
c z_{i} \in \Psi^{-1}\left(\operatorname{Fix}\left(\Gamma_{\mu_{i}} ; \gamma^{*}\right)\right)
$$

for all $c \in \mathbb{R}$. Let $w_{i}=z_{i} /\left\|z_{i}\right\|$ and $\eta_{i}=\Psi\left(w_{i}\right)$; then $\Gamma_{\eta_{i}}=\Gamma_{\mu_{i}}$. The sequence $\left\{w_{i}\right\}$ lies on the unit sphere in $\mathrm{V}$ and accumulates on the unit sphere in $T_{0} \mathrm{~S}$. By compactness there must be a subsequence converging to a point $w \in T_{0} \mathrm{~S}$. Without loss of generality (remove superfluous $z_{j}$ ) we may assume that $\left\{w_{i}\right\}$ itself converges. Then the sequence $\left\{\eta_{i}\right\}$ converges to $\eta=\Psi(w)$. For $i$ sufficiently large, $\Gamma_{\eta_{i}}$ and hence $\Gamma_{\mu_{i}}$ is conjugate to a subgroup of $\Gamma_{\eta}$, contradicting our original assumption. See figure 4.

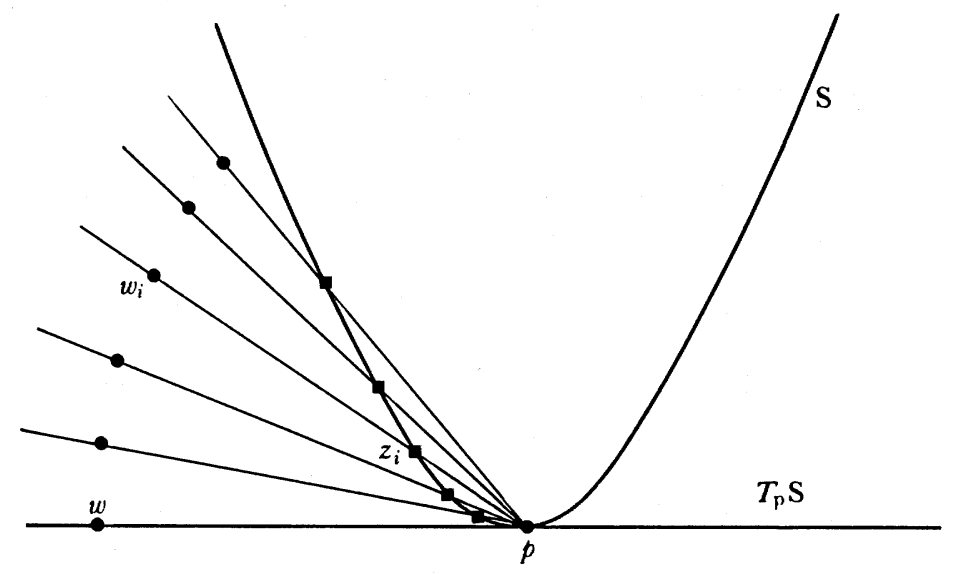

Figure 4. Proof of proposition 6.9.

To see how this result applies, let $p$ be an equilibrium point and $\lambda$ an eigenvalue of D $\mathscr{H}_{p}$ satisfying the conditions $\mathrm{H} 1$ and $\mathrm{H} 2$ of $\S 1$. Let $\Sigma$ be an isotropy subgroup of the $\Gamma \times \mathbf{S}^{1}$-action on $\mathrm{V}_{\lambda} \subset \mathrm{V}=T_{p} \mathrm{P}$, and consider a family of periodic trajectories, with symmetry groups $\Sigma$ and periods near $2 \pi /|\lambda|$, lying in a submanifold $\mathrm{S}$ of $\mathrm{P}$ containing $p$ and with $T_{p} \mathrm{~S}=\mathrm{Fix}\left(\Sigma ; \mathrm{V}_{\lambda}\right)$. (See theorem 1.1 and remark 2 of $\S 1$.) Then proposition 6.9 implies that if $u(t)$ is a periodic trajectory sufficiently close to $p$, and $\mu=\Phi(u(0))$, then $\Gamma_{\mu}$ is conjugate to a subgroup of $\Gamma_{\eta}$, for some $\eta=\Psi(v), v \in \mathrm{Fix}\left(\Sigma ; \mathrm{V}_{\lambda}\right), \mathrm{v} \neq 0$. As will be seen in the examples in $\S \S 7$ and 8 , this restricts $\Gamma_{\mu}$ considerably. In particular, if $\Gamma_{\eta}$ turns out to be a principal isotropy subgroup, a minimal isotropy subgroup for the action of $\Gamma$ on $\gamma^{*}$, then $\Gamma_{\mu}$ must be conjugate to $\Gamma_{\eta}$.

We conjecture that in fact $\Gamma_{\mu}$ is always conjugate to the corresponding $\Gamma_{\eta}$, for $u(0)$ sufficiently close to $p$, but we lack a proof. 


\section{SIMPLE EXAMPLES}

In this section we describe some simple examples that illustrate the results developed above. They are $\Gamma=\mathbf{O}(2)$ and $\mathbf{D}_{n}$ acting on $\mathbb{R}^{2} \otimes \mathbb{C} ; \mathbf{O}(n)$ on $\mathbb{R}^{n} \otimes \mathbb{C} ; \mathbf{S O}(2)$ and $\mathbf{Z}_{n}$ on $\mathbb{R}^{2}$; and SU $(2)$ on $\mathbb{R}^{4}$ and $\mathbb{R}^{4} \oplus \mathbb{R}^{2}$. These representations are chosen either because they occur frequently in applications or because of their theoretical implications.

For the purposes of this section we work only on $V_{\lambda}$ and describe the action of $\Gamma$ on $V_{\lambda}$. However, the results also apply when $\mathrm{V}_{\lambda}$ is embedded in a larger space $\mathrm{V}=T_{p} \mathrm{P}$, on which $\Gamma$ acts, provided the hypotheses of theorem 5.1 hold, that is, suitable non-resonance plus a Krein condition on all other eigenspaces. In all examples except 7.9 the space $V_{\lambda}$ is symplectically irreducible; but example 7.9 is a non-trivial ' $\Gamma$-resonance'. In several cases it is easy to find short cuts in the analysis, but we shall follow the general theory to see how it works out. A more substantial example, actions of $\mathbf{O}(3)$, is given in $\S 8$.

Example 7.1. $\mathbf{O}(2)$ acting on $\mathbb{R}^{2} \otimes \mathbb{C}$. We use the following notation for elements of $\mathbf{O}(2)$. Elements of $\mathbf{S O}(2)$ are denoted by $\theta \in \mathbb{R}(\bmod 2 \pi)$. The fip, reflection in an axis, is denoted $\kappa$. The standard action of $\mathbf{O}(2)$ on $\mathbb{R}^{2}$ is by

$$
\left.\begin{array}{l}
\theta \cdot\left[\begin{array}{l}
x \\
y
\end{array}\right]=\left[\begin{array}{rr}
\cos \theta & -\sin \theta \\
\sin \theta & \cos \theta
\end{array}\right]\left[\begin{array}{l}
x \\
y
\end{array}\right], \\
\kappa \cdot\left[\begin{array}{l}
x \\
y
\end{array}\right]=\left[\begin{array}{rr}
1 & 0 \\
0 & -1
\end{array}\right]\left[\begin{array}{l}
x \\
y
\end{array}\right] .
\end{array}\right\}
$$

This action is irreducible of real type. To obtain a symplectic action we must pass to $\mathbb{R}^{2} \otimes \mathbb{C}$. We therefore take coordinates

and complexify $(7.1)$ to obtain

$$
\left[\begin{array}{l}
z_{1} \\
z_{2}
\end{array}\right] \in \mathbb{C}^{2}
$$

$$
\begin{aligned}
& \theta \cdot\left[\begin{array}{l}
z_{1} \\
z_{2}
\end{array}\right]=\left[\begin{array}{rr}
\cos \theta & -\sin \theta \\
\sin \theta & \cos \theta
\end{array}\right]\left[\begin{array}{l}
z_{1} \\
z_{2}
\end{array}\right], \\
& \kappa \cdot\left[\begin{array}{l}
z_{1} \\
z_{2}
\end{array}\right]=\left[\begin{array}{rr}
r & 0 \\
0 & -1
\end{array}\right]\left[\begin{array}{l}
z_{1} \\
z_{2}
\end{array}\right] .
\end{aligned}
$$

Without loss of generality the $\mathbf{S}^{1}$-action is given by

$$
\phi \cdot\left[\begin{array}{l}
z_{1} \\
z_{2}
\end{array}\right]=\left[\begin{array}{l}
\mathrm{e}^{1 \phi} \\
\mathrm{e}_{1} \\
\mathrm{e}^{1 \phi} z_{2}
\end{array}\right] .
$$

The first step is to choose more suitable coordinates. Because $\mathbf{S O}(2) \times \mathbf{S}^{1}$ is abelian its action can be simultaneously diagonalized. To do this note that

$$
\left[\begin{array}{c}
1 \\
-\mathrm{i}
\end{array}\right] \text { and }\left[\begin{array}{l}
1 \\
\mathrm{i}
\end{array}\right]
$$

are simultaneous eigenvectors for all $\theta \in \mathbf{S O}(2)$, with eigenvalues $\mathrm{e}^{1 \theta}$ and $\mathrm{e}^{-1 \theta}$ respectively. They are also eigenvectors for all $\phi \in \mathbf{S}^{1}$. Define new coordinates $w_{1}, w_{2}$ on $\mathbb{C}^{2}$ by

$$
\left[\begin{array}{l}
z_{1} \\
z_{2}
\end{array}\right]=w_{1}\left[\begin{array}{c}
1 \\
-\mathrm{i}
\end{array}\right]+w_{2}\left[\begin{array}{l}
1 \\
\mathrm{i}
\end{array}\right],
$$


so that

$$
\begin{array}{ll}
z_{1}=w_{1}+w_{2}, & z_{2}=\mathrm{i}\left(w_{2}-w_{1}\right) \\
w_{1}=\frac{1}{2}\left(z_{1}+\mathrm{i} z_{2}\right), & w_{2}=\frac{1}{2}\left(z_{1}-\mathrm{i} z_{2}\right) .
\end{array}
$$

We can write $z_{j}=p_{j}+\mathrm{i} q_{j}$. The symplectic form

$$
[z, \mathrm{Z}]=\operatorname{Im}\left(z_{1} \overline{\mathrm{Z}}_{1}+z_{2} \overline{\mathrm{Z}}_{2}\right)
$$

is $\mathbf{O}(2)$-invariant, and in the $w_{j}$ coordinates takes the form

$$
[w, \mathrm{~W}]=2 \operatorname{Im}\left(w_{1} \overline{\mathbf{W}}_{1}+w_{2} \overline{\mathrm{W}}_{2}\right)
$$

The action of $\mathbf{O}(2) \times \mathbf{S}^{\mathbf{1}}$ is:

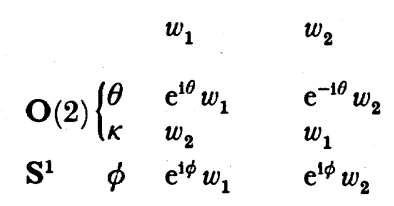

By Van Gils (1984) or Golubitsky \& Stewart (1985) the isotropy data are as shown in table 1.

TABLE 1. $\mathbf{O}(2)$ agting on $\mathbb{C} \otimes \mathbb{C}$

\begin{tabular}{lllccc}
$\begin{array}{c}\text { orbit } \\
\text { representative }\end{array}$ & \multicolumn{1}{c}{$\begin{array}{c}\text { isotropy } \\
\text { subgroup } \Sigma\end{array}$} & $\begin{array}{c}\text { fixed-point } \\
\text { subspace Fix }(\Sigma)\end{array}$ & $\begin{array}{c}\text { dim Fix }(\Sigma) \\
\text { eigenvalues } \\
\text { forced to 1 }\end{array}$ & cyclospectral? \\
$(0,0)$ & $\mathbf{O}(2) \times \mathbf{S}^{1}$ & $\{(0,0)\}$ & 0 & - & - \\
$(a, 0)$ & $\tilde{\mathbf{S O}}(2)$ & $\{(w, 0)\}$ & 2 & 2 & yes \\
$(a, a)$ & $\mathbf{Z}_{2}{ }^{c} \oplus \mathbf{Z}_{2}{ }^{c}$ & $\{(w, w)\}$ & 2 & 4 & yes \\
$(a, b)$ & $\mathbf{Z}_{2}{ }^{c}$ & $\mathbb{C}^{2}$ & 4 & 4 & yes \\
$a, b \in \mathbb{R}, a>b>0$ & & $w \in \mathbb{C}$ & & &
\end{tabular}

$$
\text { Here } \tilde{\mathbf{S O}}(2)=\{(\theta,-\theta) \mid \theta \in \mathbf{S O}(2)\}, \mathbf{Z}_{2}{ }^{\kappa}=\{(0,0),(\kappa, 0)\}, \mathbf{Z}_{2}{ }^{c}=\{(0,0),(\pi, \pi)\} .
$$

Near an equilibrium, and assuming hypotheses $\mathrm{H} 1$ and $\mathrm{H} 2$, theorem 1.1 implies that on each nearby energy level there exists at least one solution with isotropy subgroup $\widetilde{\mathbf{S O}}(2)$ and at least one with isotropy subgroup $\mathbf{Z}_{2}{ }^{{ }} \oplus \mathbf{Z}_{2}{ }^{c}$. Applying theorem 1.1 with $\Sigma=\mathbf{Z}_{2}{ }^{c}$ yields the existence of at least two solutions with isotropy subgroup containing $\mathbf{Z}_{2}{ }^{c}$, but these may just be repetitions of the two found above. This shows that theorem 1.1 may give no useful information for submaximal $\Sigma$.

We can interpret the $\widetilde{\mathbf{S O}}(2)$ solution as a rotating wave. In the spherical pendulum, example 1.3.1, it corresponds to the circular motion. The $\mathbf{Z}_{2}{ }^{{ }} \oplus \mathbf{Z}_{2}{ }^{c}$ solution is a standing wave. In the spherical pendulum it corresponds to the motion in a vertical plane.

Solutions in the $\mathbf{O}(2)$-orbits of these (having conjugate isotropy subgroups) may be described as follows. For $\widetilde{\mathbf{S O}}(2)$ there are two, which in the spherical pendulum correspond to clockwise and anticlockwise circular motion. For $\mathbf{Z}_{2}{ }^{\alpha} \oplus \mathbf{Z}_{2}{ }^{c}$ there is a circle's worth of standing waves, one for each possible reflectional symmetry, and in phase space these foliate a 2-torus. In the spherical pendulum they correspond to solutions lying in vertical planes of symmetry (see Golubitsky \& Stewart 1985, remark $(d)$, p. 128).

We can interpret the new coordinates $\left(w_{1}, w_{2}\right)$ as follows. If $w_{1}=r_{1} \mathrm{e}^{1 \theta_{1}}$ then $r_{1}$ is the amplitude and $\theta_{1}$ the phase of an anticlockwise rotating wave with isotropy subgroup $\widetilde{\mathbf{S O}}(2)$ and fixedpoint subspace $\{(w, 0)\}$. Similarly if $w_{2}=r_{2} \mathrm{e}^{\mathbf{1} \theta_{2}}$ then $r_{2}$ and $\theta_{2}$ are the amplitude and phase of a clockwise rotating wave with isotropy subgroup $\{(\theta, \theta)\}$ conjugate to $\widetilde{\mathbf{S O}}(2)$ and with fixed- 
point subspace $\{(0, w)\}$. Thus (at a linearized level) we are representing solutions as superpositions of two rotating waves. The standing waves are equal-amplitude superpositions of a wave in each direction.

Next we consider stability. The momentum mapping for $O(2) \times \mathbb{R}$ is

$$
\Phi: \mathbb{C}^{2} \rightarrow \mathrm{g}^{*} \equiv \mathbb{R}^{2},
$$

where $\mathfrak{g}$, the Lie algebra of $\mathbf{O}(2) \times \mathbb{R}$, is isomorphic to $\mathbb{R}^{2}$ with trivial Lie bracket. Because SO $(2) \times \mathbb{R}$ is abelian the coadjoint action of $\mathbf{O}(2) \times \mathbb{R}$ on $\mathrm{g}^{*}$ is trivial for $\theta \in \mathbf{S O}(2)$ and $\phi \in \mathbb{R}$. For $\kappa$ it is given by $\kappa \cdot(x, y)=(-x, y)$. All actions are trivial on the energy component of $\Phi$, corresponding to $\mathbb{R}$. Therefore $G_{\mu}$ contains $\mathbf{S O}(2)$, and $\mathfrak{g}_{\mu}=\mathfrak{g}$ for all $\mu$. This is always so when the connected component $\Gamma^{0}$ of the identity in $\Gamma$ is abelian: see the remark after proposition 4.8 .

First suppose that $\Sigma=\mathbf{Z}_{2}{ }^{\kappa} \oplus \mathbf{Z}_{2}{ }^{c}$. As noted above, $\mathfrak{g}_{\mu}=\mathfrak{g}$. Because $\boldsymbol{\Sigma}$ is finite, $\mathfrak{g}_{z}=\mathbf{0}$. Now $\mathfrak{g} / \mathfrak{g}_{\mu}=0$, and $\mathfrak{g}_{\mu} / \mathfrak{g}_{z} \otimes \mathbb{C}$ has dimension 4 . Thus the space $\mathscr{R}_{\Sigma}$ has dimension 0 . The representation of $\Sigma$ is trivially cyclospectral, and all eigenvalues of the Floquet operator $\mathrm{M}$ are forced to 1 by symmetry.

Next, suppose that $\Sigma=\widetilde{\mathbf{S O}}(2)$. Again $\mathfrak{g}_{\mu}=\mathfrak{g}$. However, $\mathfrak{g}_{z}=\left\{(x,-x) \in \mathbb{R}^{2}\right\}$. Thus $\mathfrak{g}_{\mu} / \mathfrak{g}_{z} \otimes \mathbb{C}$ is two dimensional. We compute the $\Sigma$-action on $\mathscr{R}_{\Sigma}$. Let $\chi_{k}$ denote the (complex) character of the action of $\Sigma$ on $\mathbb{C}$ in which $(\theta,-\theta)$ acts by $\mathrm{e}^{\mathrm{i} k \theta}$. On $\mathrm{V}_{\lambda}=\mathbb{C}^{2}$ the character of $\Sigma$ is

Clearly

$$
\chi\left[\mathrm{V}_{\lambda}\right]=\chi_{0}+\chi_{2}
$$

$$
\chi\left[\mathfrak{g}_{\mu} / \mathfrak{g}_{z} \otimes \mathbb{C}\right]=\chi_{0}
$$

because $\Sigma$ is abelian. By proposition $\mathbf{5 . 1 1}$

$$
\chi\left[\mathscr{R}_{\Sigma}\right]=\chi_{0}+\chi_{2}-\chi_{0}=\chi_{2} .
$$

This is a single irreducible of complex type, so $\Sigma$ is cyclospectral on $\mathscr{R}_{\Sigma}$. Hence the $\widetilde{\mathbf{S O}}(2)$ solutions are linearly stable, with two eigenvalues of $\mathrm{M}$ forced to 1 and two others on the unit circle.

Example 7.2. Non-standard actions of $\mathbf{O}(2)$. There are other irreducible representations of $\mathbf{O}(2)$. In particular if in (7.2) we change the $\theta$-action to $\left(\mathrm{e}^{k i \theta} w_{1}, \mathrm{e}^{-k i \theta} w_{2}\right)$ with $k \geqslant 2$, we again have an irreducible symplectic action of $\mathbf{O}(2) \times \mathbf{S}^{\mathbf{1}}$ on $\mathbb{C}^{2}$. The $\mathbf{O}(2)$-action has a kernel $\mathbf{Z}_{k}=\langle 2 \pi / k\rangle$, and the action induced on $\mathrm{O}(2) / \mathbf{Z}_{k} \approx \mathrm{O}(2)$ is the standard one. Therefore we can read off the behaviour from example 7.1 by pulling back each isotropy subgroup through the map $\mathbf{O}(2) \times \mathbf{S}^{1} \rightarrow \mathbf{O}(2) / \mathbf{Z}_{k} \times \mathbf{S}^{1}$. The abstract arguments, including stability assignments, are unchanged : only the interpretation of the symmetries of solutions changes. The $\mathbf{Z}_{2}{ }^{ } \oplus \mathbf{Z}_{2}{ }^{c}$ standing wave for the standard action becomes a $\mathbf{D}_{2 k}$-symmetric standing wave. The $\widetilde{\mathbf{S O}}(2)$ rotating wave becomes a rotating wave with $\mathbf{Z}_{\boldsymbol{k}}$ spatial symmetry and isotropy subgroup

$$
\widetilde{\mathbf{S O}^{k}}(2)=\{((\theta+2 \pi l) / k, \theta) \mid l=0, \ldots, k-1\} .
$$

Although the changes are mathematically trivial they are important in applications. For example, consider a two-dimensional liquid drop in $\mathbb{R}^{2}$, having an $\mathbf{O}(2)$-symmetric equilibrium state. Although this is an infinite-dimensional system the above results should remain applicable. Oscillations near this equilibrium can be expected to occur for all 'modes', that is, irreducible symplectic actions of $\mathbf{O}(2)$. The $\mathbf{D}_{2 k}$ solutions are those in which a droplet shaped 
like a (rounded) regular $k$-gon oscillates as a standing wave. The $\widetilde{\mathbf{S O}}^{k}(2)$ solutions correspond to a rotating structure with $k$-fold cyclic symmetry: like a $k$-armed spiral but close to circular form. See figure 5 .
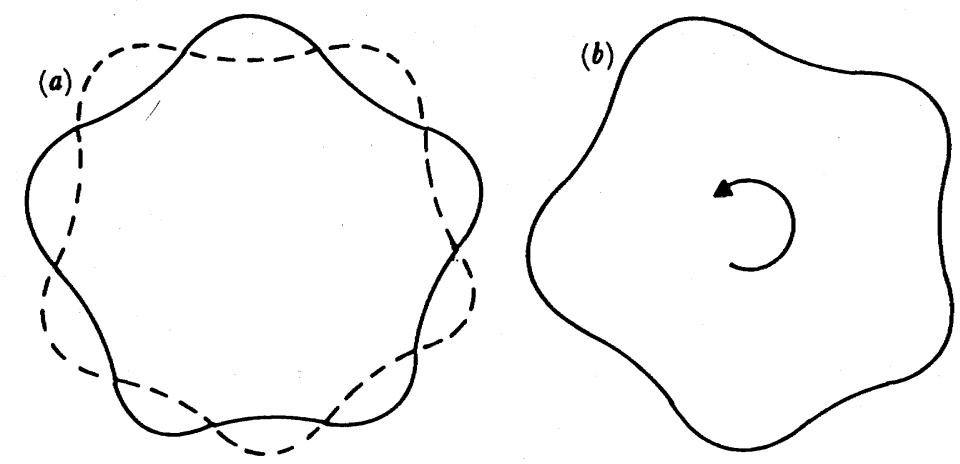

FigURE 5. Schematic illustration of symmetries of periodic solutions expected for the $k$-fold action of $\mathbf{O}(2)$.

(a) Standing wave with $\mathbf{D}_{2 k}$ symmetry; (b) rotating wave with spatial $\mathbf{Z}_{\boldsymbol{k}}$ symmetry. Here $k=\mathbf{5}$.

Such oscillations are commonly observed in a droplet of water lying on a heated surface (Aitta, personal communication). To take this analysis further requires an explicit model, and a suitable reduction to finite dimensions, but the model-independent symmetry features should, broadly speaking, be as just outlined. Other kinds of dynamic behaviour are of course possible.

Similar remarks apply to non-standard actions of $\mathbf{S O}(2), \mathbf{Z}_{n}$, and $\mathbf{D}_{n}$, provided we take care to check cyclospectrality for the appropriate actions. We shall therefore below describe only what happens for their standard actions.

Example 7.3. $\mathbf{D}_{n}$ acting on $\mathbb{R}^{2} \otimes \mathbb{C}$. Let the dihedral group $\mathbf{D}_{n}$ of order $2 n$ be realized as $\left\langle Z_{n}, \kappa\right\rangle \subset \mathbf{O}(2)$, where $Z_{n}$ is generated by $\zeta=2 \pi / n$. We take $n \geqslant 3$ for the moment because the standard action of $D_{2}$ on $\mathbb{R}^{2}$ is reducible. The standard action of $D_{n} \times S^{1}$ on $\mathbb{C}^{2}$ is obtained by restriction from (7.2) above. By Golubitsky \& Stewart (1986) the isotropy data are as in table 2 . In each case there are three maximal isotropy subgroups, with two-dimensional fixedpoint spaces, but the details depend in a minor way on $n(\bmod 4)$.

Because $\mathbf{D}_{n}$ is finite the momentum mapping provides no information that cannot be obtained more directly, but as a check we shall derive the results from the general theory. The momentum mapping for $\mathbf{D}_{n} \times \mathbb{R}$ is

$$
\begin{gathered}
\Phi: \mathbb{C}^{2} \rightarrow \mathfrak{g}^{*} \approx \mathbb{R} \\
\left(w_{1}, w_{2}\right) \mapsto \mathscr{H}\left(w_{1}, w_{2}\right) .
\end{gathered}
$$

For all $z$ we have $\mathfrak{g}=\mathfrak{g}_{\mu}, \mathfrak{g}_{z}=0$. Thus $\mathfrak{g}_{\mu} / \mathfrak{g}_{z} \otimes \mathbb{C}$ is two dimensional in all three cases, and so is $\mathscr{R}_{\Sigma}$, on which we wish to compute the $\Sigma$-action.

Suppose $\Sigma=\tilde{\mathbf{Z}}_{n}$. Let $\chi_{k}$ be the character of the action of $\Sigma$ in which $(\zeta,-\zeta)$ acts by $\mathrm{e}^{k 1 \zeta}$. Then

$$
\begin{gathered}
\chi\left[\mathrm{V}_{\lambda}\right]=\chi_{0}+\chi_{2}, \\
\chi\left[\mathfrak{g}_{\mu} / \mathfrak{g}_{z} \otimes \mathbb{C}\right]=\chi_{0}
\end{gathered}
$$

leaving

$$
\chi\left[\mathscr{R}_{\Sigma}\right]=\chi_{2}
$$

which is cyclospectral unless $n=4$.

The case $n=4$ is also exceptional in Hopf bifurcation (see Golubitsky \& Stewart 1986 and Swift 1986). 
TABLE 2. $\mathbf{D}_{n}$ Agting on $\mathbb{C} \otimes \mathbb{C}$

\begin{tabular}{|c|c|c|c|c|}
\hline $\begin{array}{l}\text { orbit } \\
\text { epresentative }\end{array}$ & $\begin{array}{l}\text { isotropy } \\
\text { subgroup } \Sigma\end{array}$ & $\begin{array}{c}\text { fixed-point } \\
\text { subspace Fix }(\Sigma)\end{array}$ & $\operatorname{dim} \operatorname{Fix}(\Sigma)$ & $\begin{array}{l}\text { number of } \\
\text { eigenvalues } \\
\text { forced to } 1 \text { cyclospectral? }\end{array}$ \\
\hline
\end{tabular}

$\begin{array}{llllll}(0,0) & \mathbf{D} \times \mathbf{S}^{1} & \{(0,0)\} & 0 & - & - \\ (a, 0) & \tilde{\mathbf{Z}}_{n}{ }^{{ }^{k}} & \{(w, 0)\} & 2 & 2 & \text { yes } \\ (a, a) & \mathbf{Z}_{2}{ }^{k} & \{(w, w)\} & 2 & 2 & \text { no } \\ (a,-a) & \mathbf{Z}_{2}^{\left({ }^{(}, \pi\right)} & \{(w,-w)\} & 2 & 2 & \text { no } \\ \left(a, w_{2}\right) & \mathbb{1} & \mathbb{C}^{2} & 4 & 2 & \text { no } \\ 0<a \in \mathbb{R}, \quad 0 \neq w_{2} \in \mathbb{C} & & w \in \mathbb{C} & & & \end{array}$

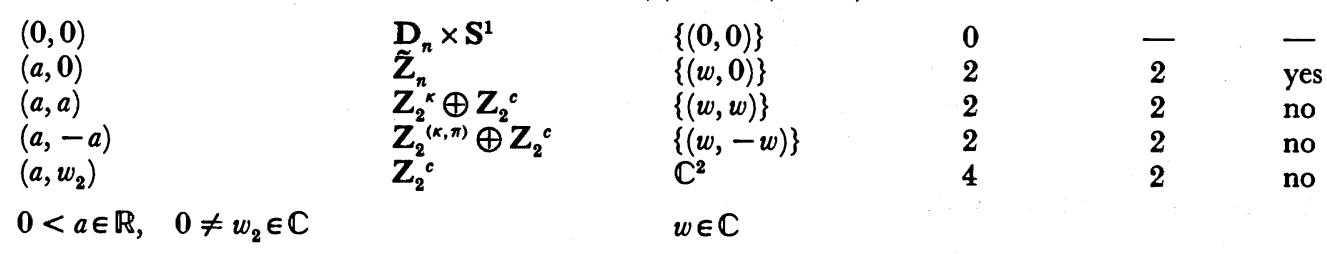

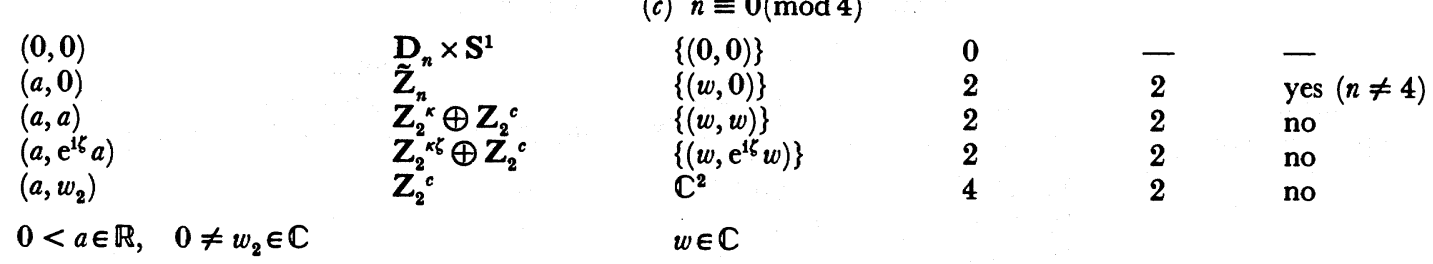

Here $\tilde{\mathbf{Z}}_{n}=\left\{(\theta,-\theta) \mid \theta \in \mathbf{Z}_{n}\right\}, \mathbf{Z}_{2}{ }^{\kappa}=\{(0,0),(\kappa, 0)\}, \mathbf{Z}_{2}{ }^{c}=\{(0,0),(\pi, \pi)\}$, and $\mathbf{Z}_{2}{ }^{(\kappa, \pi)}=\{(0,0),(\kappa, \pi)\}, \mathbf{Z}_{2}{ }^{\alpha \zeta}=\{(0,0)$, $(\kappa \zeta, 0)\}, \zeta=2 \pi / n$.

In the other two cases, where $\Sigma \approx \mathbf{Z}_{2}$ or $\mathbf{Z}_{2} \oplus \mathbf{Z}_{2}{ }^{c}$, there are two distinct characters: the trivial character $\chi_{0}$ and the non-trivial one $\chi_{1}$ in which a generator acts as $-I$. Now

$$
\chi\left[\mathrm{V}_{\lambda}\right]=\chi_{0}+\chi_{1} \quad \text { and } \quad \chi\left[\mathfrak{g}_{\mu} / \mathfrak{g}_{z} \otimes \mathbb{C}\right]=\chi_{0}
$$

so

$$
\chi\left[\mathscr{R}_{\Sigma}\right]=\chi_{1}
$$

which is of real type. Hence $\Sigma$ is not cyclospectral.

We now discuss the connection between these results and the Hénon-Heiles system, which is $D_{n}$-invariant for $n=3$. As remarked in example 1.2, the eight periodic trajectories discussed by Rod \& Churchill (1985, and references therein) can be associated with the three conjugacy classes of maximal isotropy subgroups discussed above. What we have just shown is that the $\tilde{\mathbf{Z}}_{3}$-solutions are spectrally stable, by virtue of their symmetries, and this is independent of the actual coefficients occurring in the Hénon-Heiles hamiltonian. The two types of $Z_{2}$-solution are not necessarily spectrally stable. In fact, by passing to Birkhoff normal form, it can be shown that, except at certain degeneracies and sufficiently close to equilibrium, exactly one of these two orbits of solutions is spectrally stable. In general it may be either, but for the specific hamiltonian chosen in the Hénon-Heiles system it is the orbit of $\mathbf{Z}_{2}{ }^{k}$ solutions. Similar results are derived in Churchill et al. (1983).

In the same way, for analogues of the Hénon-Heiles hamiltonian with $\mathrm{D}_{n}$ symmetry, $n \geqslant 4$, 
we find two solutions with symmetry $\tilde{\mathbf{Z}}_{n}$, and $n$ solutions each for the two kinds of $\mathbf{Z}_{2}$ symmetry. The $\tilde{\mathbf{Z}}_{n}$ solutions $(n \neq 4)$ are always spectrally stable. Such systems are described in Churchill et al. (1975). They remark there - though not in these terms - that the case of $\mathbf{D}_{2}$ symmetry is exceptional. We now show that this is a consequence of the standard representation of $\mathbf{D}_{2}$ on $\mathbb{R}^{2} \equiv \mathbb{C}$ being reducible.

Example 7.4. $\mathbf{D}_{2}$ acting on $\mathbb{R}^{2} \otimes \mathbb{C}$. The irreducible actions of $\mathbf{D}_{2}$ are on $\mathbb{R}$, not $\mathbb{R}^{2}$. There is a non-trivial kernel modulo which the action is that of $\mathbf{Z}_{2}$ or, for the trivial action, 1. Embed $\mathbf{D}_{2}$ in $\mathbf{O}(2)$ as the set of mappings $(x, y) \mapsto( \pm x, \pm y)$. The action on $\mathbb{R}^{2} \otimes \mathbb{C}$ obtained by restriction from example 7.1 is

$\begin{array}{lll} & w_{1} & w_{2} \\ 0 & w_{1} & w_{2} \\ \pi & -w_{1} & -w_{2} \\ \kappa & w_{2} & w_{1} \\ \kappa \pi & -w_{2} & -w_{1} \\ \phi & \mathbf{e}^{i \phi} w_{1} & \mathbf{e}^{i \phi} w_{2}\end{array}$

The isotropy data are as in table 3.

TABle 3. $D_{2}$ AGTing on $\mathbb{C} \otimes \mathbb{C}$

\begin{tabular}{|c|c|c|c|c|c|}
\hline $\begin{array}{c}\text { orbit } \\
\text { representative }\end{array}$ & $\begin{array}{c}\text { isotropy } \\
\text { subgroup } \Sigma\end{array}$ & $\begin{array}{c}\text { fixed-point } \\
\text { subspace Fix }(\Sigma)\end{array}$ & $\operatorname{dim} \operatorname{Fix}(\Sigma)$ & $\begin{array}{l}\text { number of } \\
\text { eigenvalues } \\
\text { forced to } 1\end{array}$ & cyclospectral? \\
\hline $\begin{array}{l}(0,0) \\
(x, x) \\
(x,-x) \\
\left(x, w_{2}\right)\end{array}$ & $\begin{array}{l}\mathbf{D}_{2} \times \mathbf{S}^{1} \\
\mathbf{Z}_{2}{ }^{k} \oplus \mathbf{Z}_{2}^{c} \\
\mathbf{Z}_{2}{ }^{k \pi} \oplus \mathbf{Z}_{2}{ }^{c} \\
\mathbf{Z}_{2}^{c}\end{array}$ & $\begin{array}{l}\{(0,0)\} \\
\{(w, w)\} \\
\{(w,-w)\} \\
\mathbb{C}^{2}\end{array}$ & $\begin{array}{l}0 \\
2 \\
2 \\
4\end{array}$ & $\begin{array}{l}- \\
2 \\
2 \\
2\end{array}$ & $\begin{array}{l}- \\
\text { no } \\
\text { no } \\
\text { no }\end{array}$ \\
\hline $\begin{array}{c}0<x \in \mathbb{R}, \quad 0 \neq w_{2} \in \mathbb{C}, \\
w_{2} \neq \pm x\end{array}$ & & $w \in \mathbb{C}$ & & & \\
\hline
\end{tabular}

Thus we get solutions for isotropy subgroups $\mathbf{Z}_{2}{ }^{\kappa} \oplus \mathbf{Z}_{2}{ }^{c}$ and $\mathbf{Z}_{2}{ }^{\kappa \pi} \oplus \mathbf{Z}_{2}{ }^{c}$. Only two eigenvalues are forced to 1 . Because all irreducible representations of $\mathbf{Z}_{2}$ are of real type, the representations are not cyclospectral. The results differ from $\mathbf{D}_{n}$ when $n \geqslant 3$ in that there are two types of solution, not three, and that linear stability is not forced on either.

Example 7.5. $\mathbf{O}(n)$ acting on $\mathbb{R}^{n} \otimes \mathbb{C}$. We take the standard action of $\mathbf{O}(n)$ on $\mathbb{R}^{n}$, which is irreducible of real type. We observe, following Golubitsky \& Stewart (1985), that the results exactly parallel $\mathbf{O}(2)$ on $\mathbb{R}^{2} \otimes \mathbb{C}$. For details see $\S 11$ of that paper. The lattice of (conjugacy classes of) isotropy subgroups is

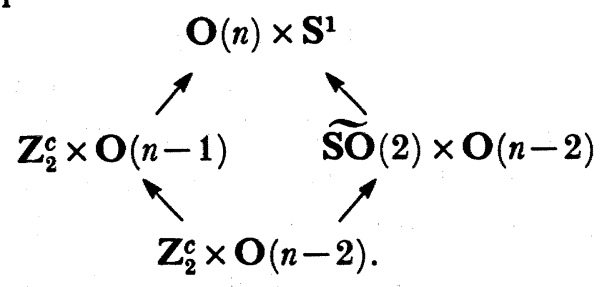

There is a rotating wave solution with isotropy $\widetilde{\mathbf{S O}}(2) \times \mathbf{O}(n-2)$ for which the Floquet operator $M$ has $2 n-2$ eigenvalues at 1 and the other two on the unit circle; and a standing wave $\mathrm{Z}_{2}{ }^{c} \times \mathbf{O}(n-1)$ with all $2 n$ eigenvalues at 1 . 
Example 7.6. SO $(2)$ acting on $\mathbb{R}^{2}$. The natural action of $\mathbf{S O}(2)$ on $\mathbb{R}^{2} \equiv \mathbb{C}$ is by $\theta \cdot z=\mathrm{e}^{1 \theta} z$. The $\mathbf{S}^{1}$-action is also by $\phi \cdot z=\mathrm{e}^{\mathrm{i} \phi} z$. The standard symplectic form is $[z, w]=\operatorname{Im}(z \bar{w})$. The isotropy data are as in table 4.

TABLE 4. SO(2) acting on $\mathbb{C}$

$\begin{array}{clcccc}\begin{array}{c}\text { orbit } \\ \text { representative }\end{array} & \begin{array}{c}\text { isotropy } \\ \text { subgroup } \Sigma\end{array} & \begin{array}{c}\text { fixed-point } \\ \text { subspace Fix }(\Sigma)\end{array} & \begin{array}{c}\text { dim } \mathbf{F i x}(\Sigma) \\ \text { number of } \\ \text { eigenvalues } \\ \text { forced to } 1\end{array} & \text { cyclospectral? } \\ 0 & \mathbf{S O}(2) \times \mathbf{S}^{1} & 0 & 0 & - & - \\ x(>0) & \mathbf{S O}(2) & \mathbb{C} & 2 & 2 & \text { yes }\end{array}$

Thus there is a solution with isotropy $\mathbf{S O}(2)$. The momentum map is

$$
\begin{aligned}
\Phi: \mathbb{C} & \mapsto \mathfrak{g}^{*} \approx \mathbb{R}^{2} \\
w & \mapsto\left(|w|^{2}, \mathscr{H}(w)\right) .
\end{aligned}
$$

The co-adjoint action is trivial so $\mathfrak{g}=\mathfrak{g}_{\mu}$. If $z \neq 0$ then $\mathfrak{g}_{z}=\{(x,-x)\} \subset \mathbb{R}^{2}$. Therefore $\mathfrak{g}_{\mu} / \mathfrak{g}_{z} \otimes \mathbb{C}$ is two dimensional, and both eigenvalues of $M$ are 1 .

This is of course obvious. By classical Floquet theory $M$ has at least one eigenvalue 1, but $M$ is symplectic, hence has determinant 1 . The existence of this solution follows from the Liapunov centre theorem, but its symmetry properties do not without further analysis.

Example 7.7. $\mathbf{Z}_{n}$ acting on $\mathbb{R}^{2}$. Let $\mathbf{Z}_{n} \subset \mathbf{S O}(2)$ act by restriction from example 7.6. The isotropy data are as in table 5 . (Note that $\tilde{\mathbf{Z}}_{\mathbf{2}}=\mathbf{Z}_{2}{ }^{c}$ )

\begin{tabular}{|c|c|c|c|c|c|}
\hline $\begin{array}{l}\text { orbit } \\
\text { representative }\end{array}$ & $\begin{array}{l}\text { isotropy } \\
\text { subgroup } \Sigma\end{array}$ & $\begin{array}{c}\text { fixed-point } \\
\text { subspace Fix }(\Sigma)\end{array}$ & $\operatorname{dim} \operatorname{Fix}(\Sigma)$ & $\begin{array}{l}\text { number of } \\
\text { eigenvalues } \\
\text { forced to } 1\end{array}$ & cyclospectral? \\
\hline $\begin{array}{l}0 \\
x(>0)\end{array}$ & ${\underset{\mathbf{Z}}{n}}_{n} \times \mathbf{S}^{1}$ & $\begin{array}{l}0 \\
\mathbb{C}\end{array}$ & $\begin{array}{l}\mathbf{0} \\
2\end{array}$ & $\overline{2}$ & yes \\
\hline
\end{tabular}

\section{TABLE 5. $\mathbf{Z}_{n}$ AGTING ON $\mathbb{C}$}

Here $\tilde{\mathbf{Z}}_{n}=\left\{(\theta,-\theta) \mid \theta \in \mathbf{Z}_{n}\right\}$.

The analysis is slightly different for the cases $n \geqslant 3, n=2$, but the end result is the same. Once more we find one solution type (also implied by the Liapunov centre theorem) with isotropy subgroup $\tilde{\mathbf{Z}}_{n}$ (not implied directly by the Liapunov centre theorem). Again both eigenvalues of $\mathrm{M}$ are 1 .

Example 7.8. SU(2) acting on $\mathbb{R}^{4}$. The geometry of this example is well known (see Cushman \& Rod 1982), but it is especially interesting. Consider $\mathbf{S U}(2)$ as the group of unit quaternions

$$
\left\{a+b i+c j+d k \mid a^{2}+b^{2}+c^{2}+d^{2}=1\right\}
$$

acting by left multiplication on $\mathbb{H} \equiv \mathbb{R}^{4}$. This is irreducible of quaternionic type.

The SU(2)-invariants are generated by the norm $N=x^{2}+y^{2}+z^{2}+t^{2}$ of elements $h=$ $x+y i+z j+t k \in \mathbb{H}$. The hamiltonian is of the form

$$
\mathscr{H}(h)=\alpha N+O\left(\|N\|^{2}\right)
$$

and $\alpha \neq 0$ for a non-degenerate equilibrium. The energy levels are 3-spheres $N=$ constant. 
The action of $\mathbf{S}^{1}$ is by a subgroup of $\mathbb{H} \backslash\{0\}$ acting on the right. Because $\mathbf{S U}(2)$ is the unique maximal compact subgroup of $\mathbb{W} \backslash\{0\}$ and all maximal tori of $\mathbf{S U}(2)$ are conjugate we may assume that

$$
\mathbf{S}^{1} \equiv\{\cos \theta+\mathrm{i} \sin \theta \mid 0 \leqslant \theta<2 \pi\}
$$

acting by right multiplication. Thus the action of $\mathbf{S U}(2) \times \mathbf{S}^{1}$ on $\mathbb{H}$ is

$$
(q, \theta) \cdot h=q h(\cos \theta+\mathrm{i} \sin \theta), \quad q \in \mathbf{S U}(2), \quad \theta \in \mathbf{S}^{\mathbf{1}}, \quad h \in \mathbb{H} .
$$

The isotropy lattice is

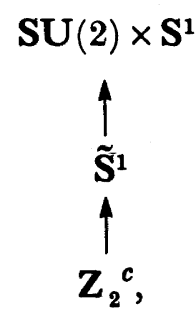

where $\mathbf{Z}_{2}{ }^{c}=\langle(-1,-1)\rangle, \tilde{\mathbf{S}}^{1}=\{(\cos \theta-\mathrm{i} \sin \theta, \cos \theta+i \sin \theta)\}$.

Clearly Fix $\left(\tilde{\mathbf{S}}^{1}\right)=\mathbb{R}\{1, \mathrm{i}\}$, which has dimension 2 , so there exists a rotating wave solution with isotropy $\widetilde{\mathbf{S}}^{1}$.

Under the $\mathbf{S U}(2)$-action the orbit of this solution fills out an invariant 3 -sphere, that is, an entire energy level. The set of periodic trajectories is the same as the Hopf fibration $\mathbf{S}^{\mathbf{1}}$-bundle over $\mathbf{S}^{2}$, see $\S 4$ and Cushman \& Rod (1982). All periodic orbits in a given energy level have the identical period.

It is easy to see that all four eigenvalues of $M$ are $\mathbf{1}$ for such solutions, so the residual representation is trivial.

Note that although this representation of $\mathbf{S U}(2)$ is of quaternionic type, an isotropy subgroup of $\mathbf{S U}(2) \times \mathbf{S}^{\mathbf{1}}$ can have a two-dimensional fixed-point subspace.

Example 7.9. SU(2) acting on $\mathbb{R}^{4} \oplus \mathbb{R}^{2}$. We include this example because an isotropy subgroup with a quaternionic isotypic block occurs. Also, it illustrates how our methods apply when there is a ' $\Gamma$-resonance', that is, when $V_{\lambda}$ is not $\Gamma$-irreducible. Suppose that $\mathbf{S U}(2)$ acts on $\mathbb{R}^{4}$ as in example 7.8 and trivially on $\mathbb{R}^{2}$. Assume that there is a $1: 1$ resonance between these representations, that is, the eigenvalues on them are the same. Then $\mathbf{S}^{1}$ acts as in example 7.8 on $\mathbb{R}^{4}$ and by the standard rotations on $\mathbb{R}^{2}$. The isotropy data are as in table 6 .

TABle 6. SU(2) Acting ON $\mathbb{H} \oplus \mathbb{R}^{2}$

$\begin{array}{clcccc}\begin{array}{c}\text { orbit } \\ \text { representative }\end{array} & \begin{array}{c}\text { isotropy } \\ \text { subgroup } \Sigma\end{array} & \begin{array}{c}\text { fixed-point } \\ \text { subspace Fix }(\Sigma)\end{array} & \operatorname{dim} \mathbf{F i x}(\Sigma) & \begin{array}{c}\text { number of } \\ \text { eigenvalues } \\ \text { forced to } 1\end{array} & \text { cyclospectral? } \\ (0,0) & \mathbf{S U}(2) \times \mathbf{S}^{1} & \{(0,0)\} & 0 & - & - \\ (x, 0) & \widetilde{\mathbf{S}}^{1} & \mathbb{R}^{2} \oplus \mathbf{0} & 4 & 4 & \text { yes } \\ (0, y) & \mathbf{S U}(2) & 0 \oplus \mathbb{R}^{2} & 2 & 2 & \text { yes } \\ (x, y) & \mathbb{1} & \mathbb{R}^{4} \oplus \mathbb{R}^{2} & 6 & 6 & \text { yes } \\ & x, y>0 . & & & \end{array}$

There are solutions with isotropy $\tilde{\mathbf{S}}^{1}$ and $\mathbf{S U}(2)$. The momentum mapping is

$$
\Phi: \mathbb{H} \oplus \mathbb{R}^{2} \rightarrow \mathfrak{g}^{*} \approx \mathfrak{s l}(2 ; \mathbb{R}) \oplus \mathbb{R},
$$$$
(q, z) \mapsto\left(\Phi_{\mathrm{H}}, \mathscr{H}(q, z)\right),
$$ 
where $\Phi_{H}(a+b i+c j+d k)$ is as in example 2 of $\S 4$. The possibilities for $\Gamma_{\mu}$ are $\mathbf{S U}(2)$ and subgroups conjugate to $\mathbf{S}^{\mathbf{1}}$.

If $\Sigma=\widetilde{\mathbf{S}}^{1}$ then in the usual notation for circle actions

$$
\chi\left[\mathrm{V}_{\lambda}\right]=\chi_{0}+\chi_{1}+\chi_{2} .
$$

If $(q, z) \in \operatorname{Fix}\left(\tilde{\mathbf{S}}^{1} ; \mathrm{V}\right)$ then an easy computation shows that $\Phi(q, z)$ has isotropy subgroup $\mathbf{S}^{1}$. The discussion in $\S 6 d$ implies that $\Gamma_{\mu}$ is conjugate to $\mathbf{S}^{1}$, and proposition 4.8 yields

$$
\chi\left[\mathfrak{g}_{\mu} / \mathfrak{g}_{z} \otimes \mathbb{C}\right]=\chi_{0}, \quad \chi\left[\mathfrak{g} / \mathfrak{g}_{\mu}\right]=\chi_{2},
$$

whence $\chi\left[\mathscr{R}_{\Sigma}\right]=\chi_{1}$ and the solution is spectrally stable.

If $\Sigma=\mathbf{S U}(2)$ then we let $\chi_{\mathrm{H}}$ be the character of the standard representation on $\mathbb{H}$ and $\chi_{0}$ be the trivial character. We have

and

$$
\chi\left[\mathrm{V}_{\lambda}\right]=\chi_{\mathrm{H}}+\chi_{0}
$$$$
\chi\left[\mathscr{R}_{\Sigma}\right]=\chi_{\mathrm{H}} .
$$

This is a non-repeated quaternionic isotypic block, hence the representation is cyclospectral and the solution is spectrally stable.

If $\Sigma=1$ then the formula of corollary 4.7 shows that all the eigenvalues of the Floquet operator are equal to 1 .

\section{SPHERICAL SYMMETRY: THE GROUP $\mathbf{O}(3)$}

In this final section we discuss the existence and stability of periodic trajectories in hamiltonian systems with spherical symmetry - the orthogonal group $\mathbf{O}(3)$ in $\mathbb{R}^{3}$ - near equilibria fixed by the $\mathbf{O}(3)$-action. The reader may like to keep in mind the example of a vibrating liquid drop or bubble (Lamb 1932). This is an infinite-dimensional system, but the results described here should still be applicable.

\section{(a) Existence of periodic trajectories}

Let $\mathrm{U}_{l}(l=0,1, \ldots)$ denote the space of spherical harmonics of order $l$ with its natural action of $\mathbf{O}(3)$. This is a real irreducible representation, so by theorem 2.1 the spaces $\mathrm{V}_{l}=\mathrm{U}_{l} \oplus \mathrm{U}_{l} \approx \mathrm{U}_{l} \otimes \mathbb{C}$, with induced $\mathbf{O}(3)$-actions and standard symplectic forms, are real irreducible symplectic representations. Let $\mathscr{H}$ be an $\mathbf{O}(3)$-invariant hamiltonian satisfying, at an equilibrium $p$, the hypotheses $\mathrm{H} 1$ and $\mathrm{H} 2$ of theorem 1.1, with $\mathrm{V}_{\lambda} \approx \mathrm{V}_{l}$ for some $l$. The latter condition holds generically. The $\mathbf{S}^{1}$-action given by the linearized flow on $V_{\lambda}$ is isomorphic to that induced on $\mathrm{V}_{l}$ from the action by multiplication by unit-circle complex numbers on $\mathbb{C}$. The isotropy subgroups of the resulting $\mathbf{O}(3) \times \mathbf{S}^{1}$-action that have two dimensional fixed-point subspaces are listed in table 7, which is taken from Golubitsky \& Stewart (1985) table 14.1. Our restriction to natural representations of $\mathbf{O}(3)$ on spherical harmonics means that, in their terminology, we consider only the 'plus representation' for even $l$ and the 'minus representation' for odd $l$. We have also made a correction: the isotropy subgroups in the next to last row of their table 14.1 are conjugate to subgroups of those in the last row, and hence should be omitted.

As usual $\mathrm{K}$ is $\Sigma \cap \Gamma$ and $\mathrm{H}$ is the projection of $\Sigma$ into $\mathbf{O}(3)$. The symmetry group $\Sigma$ can be reconstructed from the pair $(\mathrm{H}, \mathrm{K})$ by using a twist map, see $\S 4 c$. If the twist is trivial we have 
Table 7. Isotropy subgroups of $\mathbf{O}(3) \times \mathbf{S}^{1}$ agting on $V_{l}$, With tWo-dimensional FIXED-POINT SUBSPAGES

\begin{tabular}{|c|c|c|c|c|}
\hline$\Sigma$ & $\mathbf{H}$ & $\mathbf{K}$ & $l$ & $\begin{array}{c}\text { number of } \\
\text { eigenvalues }=1\end{array}$ \\
\hline \multicolumn{5}{|c|}{ evén $l$} \\
\hline $\mathbf{O}(2)$ & $\mathbf{O}(2)$ & $\mathbf{O}(2)$ & all even & 6 \\
\hline & SO $(2)$ & $\mathbf{Z}_{k}$ & all even & 4 \\
\hline ॥ & 0 & 0 & $\begin{array}{c}6,10,12,16,18,20,22,24 \\
26,28,32,34,38,44\end{array}$ & 8 \\
\hline$\underset{\widetilde{Q}}{\mathbb{Q}}$ & 0 & 0 & $4,6,8,10,14$ & 8 \\
\hline$\tilde{\mathbb{Q}}$ & $\mathbb{O}$ & $\pi$ & $6,10,12,14,16,20$ & 8 \\
\hline$\tilde{\tilde{\mathbb{\pi}}}$ & $T$ & $\mathbf{D}_{2}$ & $2,4,6$ & 8 \\
\hline$\tilde{\mathbf{D}}_{2 k}$ & $\mathbf{D}_{2 k}$ & $\mathbf{D}_{\boldsymbol{k}}$ & $\frac{1}{2} l<k \leqslant l$ & 8 \\
\hline \multicolumn{5}{|c|}{ odd $l$} \\
\hline \multirow{3}{*}{$\begin{array}{l}\left(\mathbf{O}(2) \oplus \mathbf{Z}_{2}^{c}\right)^{\sim k} \\
\left(\mathbf{S O}(2) \oplus \mathbf{Z}_{2}^{c}\right)^{\sim k} \\
k=1, \ldots, l \\
\left(\mathbb{1} \oplus \mathbf{Z}_{2}^{c}\right)^{\sim}\end{array}$} & $\mathbf{O}(2) \oplus \mathbf{Z}_{2}{ }^{c}$ & $\mathbf{O}(2)^{-}$ & all odd & 6 \\
\hline & $\mathbf{S O}(2) \oplus \overline{\mathbf{Z}}_{2}^{c}$ & $\mathbf{Z}_{k}^{-}$ & all odd & 4 \\
\hline & $\mathbb{v} \oplus \mathbf{Z}_{2}{ }^{c}$ & $\rrbracket$ & $\begin{array}{r}21,25,27,31,33,35,37 \\
39,41,43,47,49,53,59\end{array}$ & 8 \\
\hline$\left(\mathbb{O} \oplus Z_{2}{ }^{c}\right)^{\sim 1}$ & $\mathbb{1} \oplus \mathbf{Z}_{2}{ }^{c}$ & $\mathbb{1}$ & $9,13,15,17,19,23$ & 8 \\
\hline 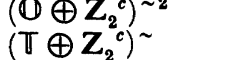 & 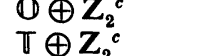 & $\mathbb{O}^{-}$ & $\begin{array}{l}3,7,9,11,13,17 \\
5,7,9\end{array}$ & $\begin{array}{l}8 \\
8\end{array}$ \\
\hline$\left(\mathbf{D}_{2 k} \oplus \mathbf{Z}_{2}^{c}\right)^{\sim}$ & $\mathbf{D}_{k} \oplus \mathbf{Z}_{2}^{2}{ }^{c}$ & $\mathbf{D}_{k}^{2}$ & $1<\frac{1}{2} l<k \leqslant l$ & 8 \\
\hline
\end{tabular}

$\Sigma=\mathrm{H}$; otherwise we write $\Sigma=\tilde{\mathrm{H}}$. A superscript is appended to the tilde when necessary to distinguish different $\Sigma$ with the same $\mathrm{H}$. When $l$ is even $-\mathrm{I} \in \mathbf{O}(3)$ acts trivially, so both $\mathrm{H}$ and $\mathrm{K}$ contain $\mathbf{Z}_{2}{ }^{c}=\{ \pm I\}$. However, because it enters trivially it is omitted in the table. When $l$ is odd $-I$ composed with phase shift $\pi$ always belongs to $\Sigma$, so $\mathrm{H}$ contains $\mathbf{Z}_{2}^{c}$ when $l$ is odd, but $\mathrm{K}$ does not. The notation used for subgroups of $\mathbf{O}(3)$ is the same as that in Golubitsky \& Stewart (1985). In particular $\mathbb{T}, \mathbb{O}, \mathbb{Q}$ are the tetrahedral, octahedral, and icosahedral subgroups of $\mathbf{S O}(3)$. The groups $\mathbf{O}(2), \mathbf{S O}(2), \mathbb{T}, \mathbb{O}, \mathbb{1}, \mathbf{D}_{k}$, and $\mathbf{Z}_{k}$ are all subgroups of $\mathbf{S O}(3)$, and the superscript - denotes an isomorphic but non-conjugate subgroup of $\mathbf{O}(3)$.

From theorem 1.1 and remark 2 in $\S 1$ we know that for each such isotropy subgroup $\Sigma$ there is, passing through $p$, a smooth family of periodic trajectories of the nonlinear flow with periods near $2 \pi /|\lambda|$ and symmetry groups $\Sigma$. For $l=0,1,2$ these solutions are shown in figure 6 .

In general there will be other maximal isotropy subgroups, with higher-dimensional fixedpoint subspaces, giving rise to other periodic trajectories. However, these subgroups have not yet been classified and are not considered here.

We can also prove the existence of some periodic trajectories with submaximal isotropy groups. Suppose that $l$ is even and consider $\Sigma=\tilde{\mathbf{D}}_{2 k} \subset \mathbf{O}(3) \times \mathbf{S}^{1}$ when $\frac{1}{4} l<k \leqslant \frac{1}{3} l$. From $(8.8,8.9)$ below Fix $\left(\tilde{\mathbf{D}}_{2 k}\right)$ is four-dimensional, whence by theorem 1.1 in each energy level near $p$ there exist at least two periodic trajectories with symmetry group containing $\tilde{\mathbf{D}}_{2 k}$. Now $\operatorname{Fix}\left(\tilde{\mathbf{D}}_{2 k}\right) \supset \operatorname{Fix}\left(\tilde{\mathbf{D}}_{4 k}\right)$ which has dimension 2, so (precisely) one of these two trajectories has symmetry group $\tilde{\mathbf{D}}_{4 k}$. However, no other subgroup conjugate to one listed in table 8.1 contains $\tilde{\mathbf{D}}_{2 k}$, so there are no other two dimensional fixed-point subspaces lying in Fix $\left(\tilde{\mathbf{D}}_{2 k}\right)$. Therefore there must also be a periodic trajectory with period near $2 \pi /|\lambda|$ and submaximal symmetry 


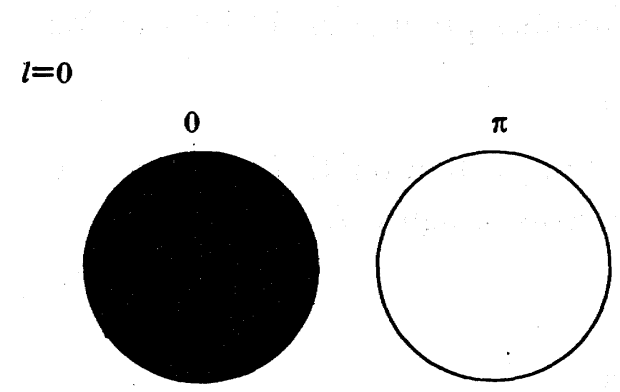

$\mathbf{O}(3)$ standing wave

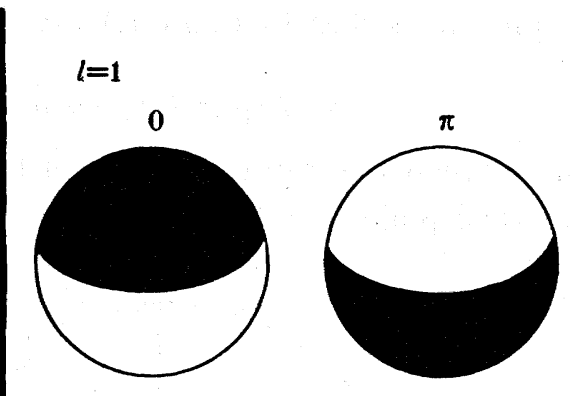

$O(2)$ standing wave

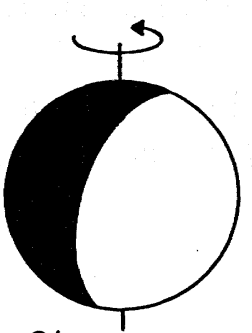

$\widetilde{\mathrm{SO}}(2)$ rotating wave

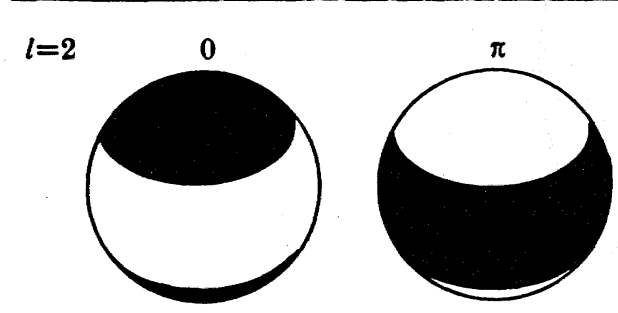

$O(2)$ standing wave

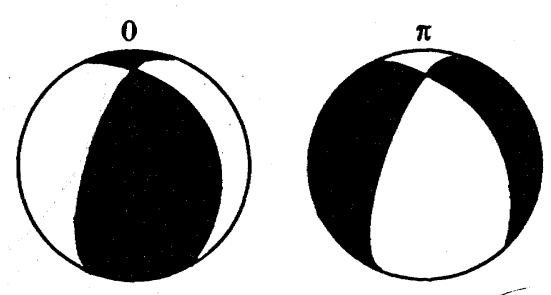

$\tilde{D}_{1}$ standing wave

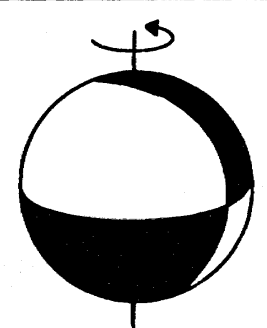

$(\mathrm{SO}(2))^{\sim 1}$ rotating wave

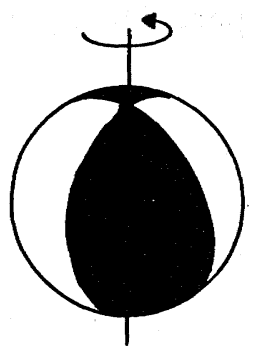

$(\mathrm{SO}(2))^{\sim 2}$

rotating wave
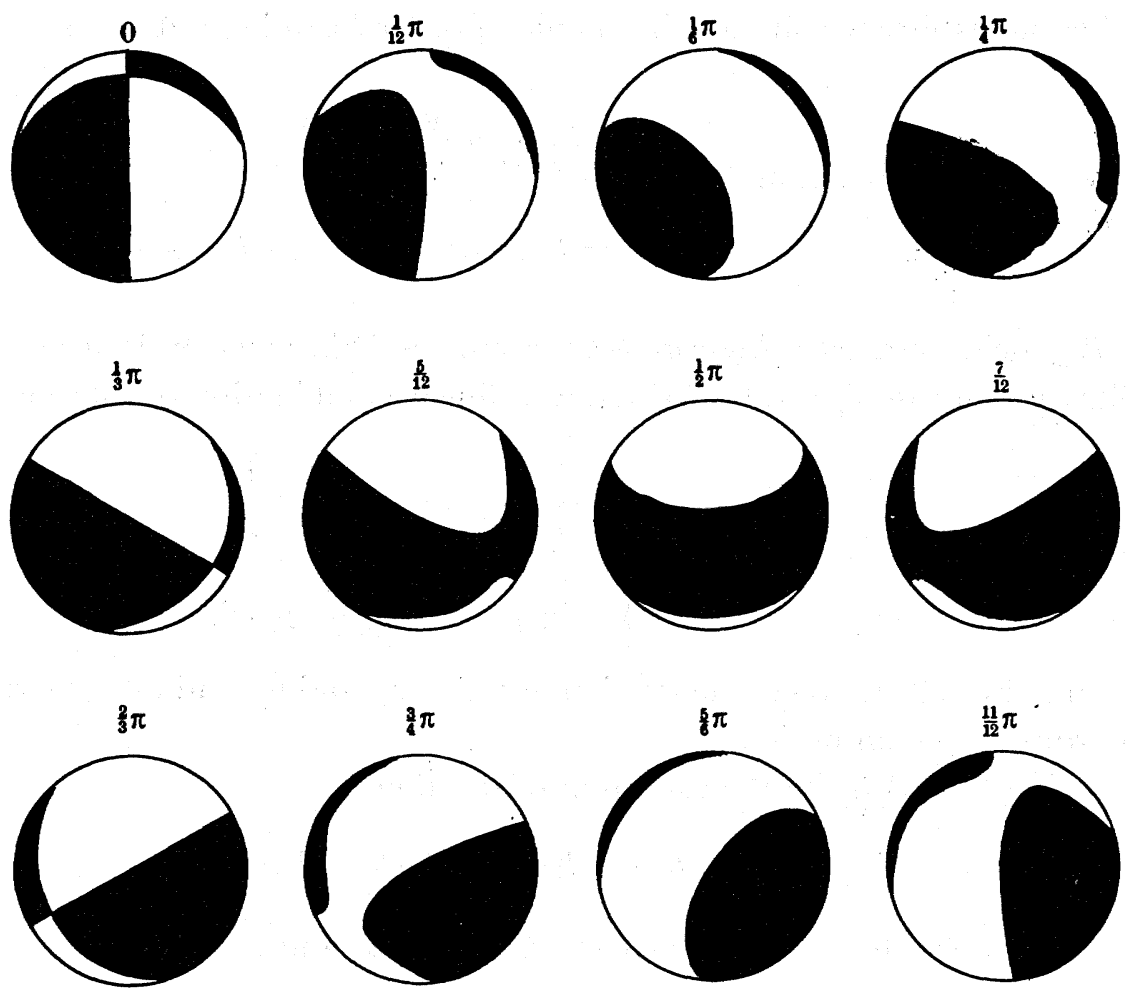

$\tilde{\mathrm{T}}$ discrete rotating wave

Figure 6. Periodic solutions (of period $2 \pi$ ) corresponding to isotropy subgroups $\Sigma \subset \mathbf{O}(3)$ with $\operatorname{dim} F i x(\Sigma)=2$, for $l=0,1,2$, for the liquid drop. Note that for the tetrahedral solution $\Sigma=\tilde{\pi}$ a $\frac{2}{3} \pi$ phase shift has the same effect as a $\frac{2}{3} \pi$ rotation. 
group $\tilde{\mathbf{D}}_{2 k}$. This example shows that theorem 1.1 can sometimes provide useful information for submaximal $\Sigma$.

(b) Eigenvalues forced to 1

First we give a more explicit description of part of the $\mathbf{O}(3)$-action on $V_{l}$. Let $0 \leqslant \alpha \leqslant \pi$, $0 \leqslant \beta<2 \pi$ denote spherical polar coordinates on the sphere as in figure 7 .

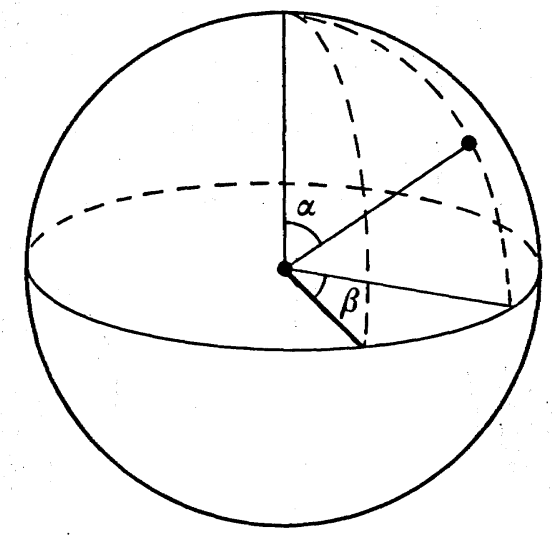

Figure 7. Spherical polar coordinates.

Then an arbitrary spherical harmonic of order $l$ can be written as

where $z_{m} \in \mathbb{C}, z_{-m}=\bar{z}_{m}$, and

$$
\sum_{m=-l}^{l} z_{m} Y_{l, m}(\alpha, \beta)
$$

$$
Y_{l, m}(\alpha, \beta)=P_{l, m}(\cos \alpha) \mathrm{e}^{\mathrm{i} m \beta} \quad(-l \leqslant m \leqslant l),
$$

the $P_{l, m}$ being associated Legendre polynomials, see Whittaker \& Watson (1927). Similarly an arbitrary point in $V_{l}$ can be written as a linear combination of rotating spherical harmonics

where $z_{m} \in \mathbb{C}$ and

$$
\operatorname{Re}\left\{\sum_{m=-l}^{l} z_{m} Y_{l, m}(\alpha, \beta, t)\right\},
$$

$$
Y_{l, m}(\alpha, \beta, t)=Y_{l, m}(\alpha, \beta) \mathrm{e}^{i t}
$$

Note that in (8.3) we relax the restriction $z_{-m}=\bar{z}_{m}$ and instead take the real part. The $z_{m}$ form a coordinate system on $\mathrm{V}_{l}$.

Let $\mathbf{S O}(2) \subset \mathbf{O}(3)$ be the subgroup of rotations

$$
\theta:(\alpha, \beta) \mapsto(\alpha, \beta+\theta) \quad \theta \in[0,2 \pi)
$$

and let $\mathbf{O}(2)$ be the subgroup generated by $\mathbf{S O}(2)$ and

$$
\kappa:(\alpha, \beta) \mapsto(\pi-\alpha,-\beta)
$$

Then the action of $\mathbf{O}(2)$ on $V_{l}$ is given by

$$
\begin{gathered}
\theta \cdot\left(z_{-l}, \ldots, z_{l}\right)=\left(\mathrm{e}^{-\mathrm{i} l \theta} z_{-l}, \ldots, \mathrm{e}^{\mathrm{i} r \theta} z_{r}, \ldots, \mathrm{e}^{\mathrm{i} l \theta} z_{l}\right) \\
\kappa \cdot\left(z_{-l}, \ldots, z_{l}\right)=\left(z_{l}, \ldots,(-1)^{l-r} z_{-r}, \ldots, z_{-l}\right) .
\end{gathered}
$$


The rest of the $\mathbf{O}(3)$-action is more difficult to describe, but we do not need it explicitly. The $\mathbf{S}^{1}$-action is

$$
\phi \cdot\left(z_{-l}, \ldots, z_{l}\right)=\left(\mathrm{e}^{\mathrm{I} \phi} z_{-l}, \ldots, \mathrm{e}^{\mathrm{i} \phi} z_{l}\right)
$$

We now calculate the momentum isotropy subgroup $\Gamma_{\mu}$ for each $\Sigma$. The Lie algebra $\mathfrak{o}(3)$ of $\mathbf{O}(\mathbf{3})$ is three dimensional, and the action of $\mathbf{O}(3)$ on $\mathfrak{v}(3)$ is generated by the standard action of $\mathbf{S O}(3)$ on $\mathbb{R}^{3}$ and the trivial action of $Z_{2}{ }^{c}$. Thus the only possible momentum isotropy subgroups are $\mathbf{O}(3)$ and subgroups conjugate to $\mathbf{S O}(2) \oplus \mathbf{Z}_{2}{ }^{c}$.

Proposition 8.1. If $\Sigma=(\mathbf{S O}(2))^{\sim k}$ or $\left(\mathbf{S O}(2) \oplus \mathbf{Z}_{2}{ }^{c}\right)^{\sim k}$ then $\Gamma_{\mu}$ is conjugate to $\mathbf{S O}(2) \oplus \mathbf{Z}_{2}{ }^{c}$. Otherwise $\Gamma_{\mu}=\mathbf{O}(3)$.

Proof. By proposition $4.9 \Gamma_{\mu}$ must contain $\mathrm{H}$, the projection of $\Sigma$ into $\mathrm{O}(3)$. From table 8.1 the only $\Sigma$ with $\mathrm{H} \subset \mathbf{S O}(2) \oplus \mathbf{Z}_{2}{ }^{c}$ are $(\mathbf{S O}(2))^{\sim k}$ and $\left(\mathbf{S O}(2) \oplus \mathbf{Z}_{2}^{c}\right)^{\sim k}$. Thus periodic solutions with all other (listed) symmetry types must have zero angular momentum. If $\Sigma$ is $(\mathbf{S O}(2))^{\sim k}$ or $\left(\mathbf{S O}(2) \oplus \mathbf{Z}_{2}{ }^{c}\right)^{\sim k}$ then $\operatorname{Fix}(\Sigma)=\left\{z_{j}=0 \mid j \neq-k\right\}$. The component of the $\Gamma$-momentum mapping coming from the $\mathbf{S O}(2)$-action is $\sum_{r=-l}^{l} r\left|z_{r}\right|^{2}$, see example 1 of $\S 4 a$, and is therefore non-zero if $\left(z_{-l}, \ldots, z_{l}\right) \in \mathrm{Fix}(\Sigma)$. By the discussion in $\S 6 d$ the momentum isotropy subgroups $\Gamma_{\mu}$ for $(\mathbf{S O}(2))^{\sim k}$ and $\left(\mathbf{S O}(2) \oplus \mathbf{Z}_{2}{ }^{c}\right)^{\sim k}$ are conjugate to $\mathbf{S O}(2) \oplus \mathbf{Z}_{2}{ }^{c}$.

By this proposition and theorem 4.5 it is a straightforward exercise to compute the number of Floquet multipliers of a periodic trajectory with symmetry group $\Sigma$ that are forced to 1 . The results are given in the last column of table 7.

\section{(c) The residual Floquet operator}

To take the stability analysis further we must proceed case by case.

(i) $\mathbf{O}(2)$ and $\left(\mathbf{O}(2) \oplus \mathbf{Z}_{2}^{c}\right)^{\sim}$

The analysis is essentially the same in both cases, so we consider only $\mathbf{O}(2)$, which occurs for even $l$. The action of $\mathbf{O}(2)$ on $\mathrm{V}_{l}$ is given by $(8.5,8.6)$. The complex character of this representation is $\Sigma_{0}^{l} \chi_{r}$, where $\chi_{r}$ is the character of the representation on $\left\{z_{j}=0 \mid j \neq \pm r\right\}$. The $\chi_{r}$ are irreducible and pairwise non-isomorphic. Note that the factors $(-1)^{l-r}$ in $(8.6)$ do not affect the isomorphism type of the representation. To calculate the residual representation $\mathscr{R}_{\Sigma}$ we use proposition 6.8. Let $g$ be the Lie algebra of $\mathbf{O}(3) \times \mathbb{R}$ and $g_{z}$ that of $\mathbf{O}(2)$. By the above, $\chi\left[\mathrm{V}_{\lambda}\right]=\Sigma_{0}^{l} \chi_{r}$, while $\chi\left[\mathfrak{g} / \mathfrak{g}_{\mu}\right]=0$ and $\chi\left[\mathfrak{g}_{\mu} / \mathfrak{g}_{z} \otimes \mathbb{C}\right]=\chi[\mathfrak{g} \otimes \mathbb{C}]-\chi\left[\mathfrak{g}_{z} \otimes \mathbb{C}\right]$. Defining $\chi_{r}$ as above and letting $\chi_{0}{ }^{-}$be the non-trivial complex one-dimensional character of $\mathbf{O}(2)$ we have $\chi[\mathfrak{g} \otimes \mathbb{C}]=\chi_{0}+\chi_{0}{ }^{-}+\chi_{1}$ and $\chi\left[g_{z} \otimes \mathbb{C}\right]=\chi_{0}{ }^{-}$. Thus the character of the residual representation is $\Sigma_{2}^{l} \chi_{r}$. This is cyclospectral only if $l \leqslant 1$. The residual Floquet operator behaves as an element of $[\operatorname{Sp}(2 ; \mathbb{R})]^{l-1}$, the product of $l-1$ copies of $\mathbf{S p}(2 ; \mathbb{R})$.

(ii) $(\mathbf{S O}(2))^{\sim k}$ and $\left(\mathbf{S O}(2) \oplus \mathbf{Z}_{2}^{c}\right)^{\sim k}$

Again we consider only $(\mathbf{S O}(2))^{\sim k}$ explicitly, where $k=1, \ldots, l$. If $\psi$ parametrizes $(\mathbf{S O}(2))^{\sim k}$ its action on $\mathrm{V}_{l}$ is

$$
\psi \cdot\left(z_{-l}, \ldots, z_{r}, \ldots, z_{l}\right)=\left(\mathrm{e}^{1(-l+k)} z_{-l}, \ldots, \mathrm{e}^{1(r+k)} z_{r}, \ldots, \mathrm{e}^{1(l+k)} z_{l}\right)
$$

so that, in the obvious notation,

$$
\chi\left[\mathrm{V}_{\lambda}\right]=\sum_{-l+k}^{l+k} \chi_{r}
$$


In proposition 6.8 we must take $g$ to be the Lie algebra of $\mathbf{O}(3) \times \mathbb{R}$, and $g_{\mu}$ that of $\mathbf{S O}(2) \times \mathbb{R}$, and $\mathrm{g}_{z}$ that of $(\mathbf{S O}(2))^{\sim k}$. Clearly

$$
\begin{gathered}
\chi\left[\mathrm{g} / \mathfrak{g}_{\mu}\right]=\chi_{1}, \\
\chi\left[\mathfrak{g}_{\mu} / \mathfrak{g}_{z} \otimes \mathbb{C}\right]=\chi\left[\mathfrak{g}_{\mu} \otimes \mathbb{C}\right]-\chi\left[\mathfrak{g}_{z} \otimes \mathbb{C}\right]=2 \chi_{0}-\chi_{0}=\chi_{0} .
\end{gathered}
$$

Thus the residual representation has character

$$
\chi_{-l+k}+\ldots+x_{-1}+\chi_{2}+\ldots+\chi_{k+l}
$$

This is cyclospectral if and only if $k=l$ or $l-1$. In general the residual Floquet operator behaves as an element of

$$
[\mathbf{U}(1 ; \mathbb{C})]^{l+k} \times[\mathbf{U}(1,1 ; \mathbb{C})]^{l-k+1}
$$

(iii) $\tilde{\mathbf{D}}_{2 k}$ and $\left(\mathbf{D}_{k} \oplus \mathbf{Z}_{2}^{c}\right)^{\sim}$

We consider $\tilde{\mathbf{D}}_{2 k}$. This is generated by $\kappa$ and the element $\psi$ obtained by composing $\pi / k \in \mathbf{S O}(2)$ with $\pi \in \mathbf{S}^{1}$. We have

$$
\left.\begin{array}{rl}
\psi \cdot\left(z_{-l}, \ldots, z_{l}\right) & =\left(-\mathrm{e}^{-\mathrm{ill} / k} z_{-l}, \ldots,-\mathrm{e}^{\mathrm{i} r \pi / k} z_{r}, \ldots,-\mathrm{e}^{\mathrm{i} l \pi / k} z_{l}\right) \\
& =\left(\mathrm{e}^{\mathrm{i}(k-l) \pi / k} z_{-l}, \ldots, \mathrm{e}^{\mathrm{i}(k+r) \pi / k} z_{r}, \ldots, \mathrm{e}^{\mathrm{i}(k+l) \pi / k} z_{l}\right)
\end{array}\right\}
$$

Denote by

$\chi_{k-r}, r \neq 0, k$, the complex character of the four-dimensional (over $\mathbb{R}$ ) irreducible symplectic representation of $\tilde{\mathbf{D}}_{2 k}$ on the space spanned by $z_{-r}$ and $z_{r}$;

$\chi_{k}$, the character of the two-dimensional irreducible symplectic representation of $\tilde{\mathbf{D}}_{2 k}$ on the span of $z_{0}$;

$\chi_{0}{ }^{+}$the character of the two-dimensional trivial representation on the span of $z_{-k}+(-1)^{k} z_{k}$

$\chi_{0}{ }^{-}$the character of the two-dimensional irreducible symplectic representation of $\tilde{\mathbf{D}}_{2 k}$ on the span of $z_{-k}-(-1)^{k} z_{k}$.

Note that $\chi_{k-r_{1}}=\chi_{k-r_{2}}$ if and only if $r_{1} \equiv r_{2}(\bmod k)$. Then

$$
\chi\left[\mathrm{V}_{\lambda}\right]=\chi_{0}^{-}+\chi_{0}^{+}+\sum_{\substack{r=0 \\ r \neq k}}^{l} \chi_{k-r}
$$

Clearly $\mathfrak{g}_{z}=0$, while $\mathfrak{g}_{\mu}=\mathfrak{g}$ is the Lie algebra of $\mathbf{O}(3) \times \mathbb{R}$. Thus $\chi\left[\mathfrak{g} / \mathfrak{g}_{\mu}\right]=0$, and $\chi\left[\mathfrak{g}_{\mu} / \mathfrak{g}_{z} \otimes \mathbb{C}\right]$ is the character of the complexification of the standard action of $\mathbf{D}_{2 k}$ on $\mathbb{R}^{3} \oplus \mathbb{R}$, that is, $\chi_{1}+\chi_{0}{ }^{-}+\chi_{0}{ }^{+}$. Thus the residual representation is

which equals

$$
\begin{gathered}
\sum_{\substack{r=0 \\
r \neq k, k-1}}^{l} \chi_{k-r}, \\
\chi_{1}+2 \sum_{r=2}^{l-k} \chi_{k-r}+\sum_{r=l-k+1}^{k} \chi_{r} .
\end{gathered}
$$

Hence the residual Floquet operator behaves as an element of

$$
[\mathbf{S p}(2 ; \mathbb{R})]^{\min (l-1,2 k-l+1)} \times[\mathbf{S p}(4 ; \mathbb{R})]^{l-k+1} .
$$




\section{(iv) The others}

We do not give detailed calculations for the remaining isotropy subgroups with twodimensional fixed-point subspaces, namely those involving $\mathbb{\mathbb { Q }} \mathbb{\mathbb { O }}$, and $\mathbb{T}$. However, note that all symplectic irreducible representations of $\mathbb{0}$ and $\mathbb{O}$ are real, hence so are those of $\left(\tilde{\mathbb{O}}, \mathbb{\square} \oplus \mathbf{Z}_{2}{ }^{c}\right)^{\sim}$, $\left(\mathbb{O} \oplus \mathbf{Z}_{2}^{c}\right)^{\sim 1}$, and $\left(\mathbb{O} \oplus \mathbf{Z}_{2}^{c}\right)^{\sim 2}$. Thus the residual representations can never be cyclospectral. We therefore expect that it is possible for the corresponding periodic trajectories to be hyperbolic (for suitable choices of the hamiltonian). On the other hand, the tetrahedral group $\mathbb{T}$ does have a pair of two-dimensional complex symplectic irreducibles. The only other symplectic irreducibles are the trivial one and a six-dimensional real irreducible. When $l=2$ the calculation of the number of eigenvalues equal to 1 shows that the residual representation must be two dimensional. Because it cannot be trivial it must be complex and hence cyclospectral.

\section{TABLE 8. Residual Floquet operators for $l \leqslant 3$}

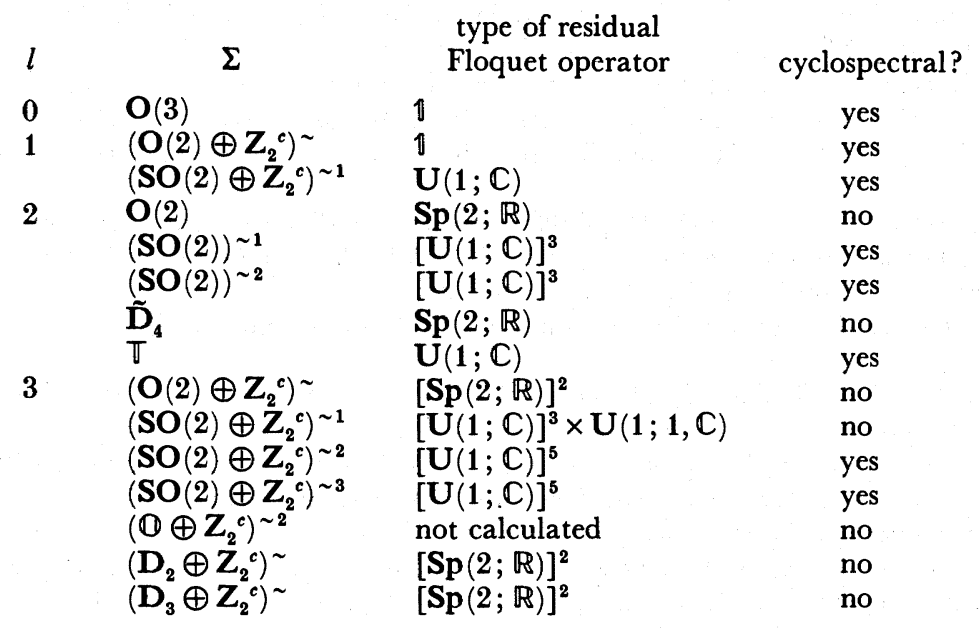

The results of these calculations are summarized, for $l=0,1,2$, and 3 , in table 8 . In these low dimensions many of the periodic trajectories are forced to be spectrally stable. This ceases to be true as $l$ increases, though the two 'fastest spinning' rotating waves are always spectrally stable. Nevertheless, in all cases the possible behaviour of the Floquet multipliers is severely constrained by the group action, and these constraints are given precisely by the type of the residual Floquet operator, indicated in the third column of the table.

We are grateful to Robert MacKay for introducing us to Krein theory, and to Marty Golubitsky for telling us of unpublished work of Xianwu Zeng. The work of J. M. and R. S. was partly supported by a SERC Research Grant, and that of M.R. by an SERC Advanced Research Fellowship. 


\section{REFERENCES}

Abraham, R. \& Marsden, J. E. 1978 Foundations of mechanics, 2nd edn. Reading: Benjamin/Cummings.

Abraham, R., Marsden, J.E. \& Ratiu, T. 1983 Manifolds, tensor analysis, and applications. Reading: AddisonWesley.

Adams, J. F. 1969 Lectures on Lie groups. New York: Benjamin. (Midway Reprint, University of Chicago Press, 1982.)

Alexander, J. \& Yorke, J. A. 1978 Global bifurcation of periodic orbits. Am. J. Math. 100, 263-292.

Arms, J., Fisher, A. \& Marsden, J. E. 1975 Une approche symplectique pour des théorèmes de decomposition en géométrie ou relativité générale. C. R. acad. Sci., Paris 281, 517-520.

Arnold, V. I. 1978 Mathematical methods of classical mechanics. New York: Springer.

Arnold, V. I. \& Avez, A. 1968 Ergodic problems of classical mechanics. New York: Benjamin.

Churchill, R. C., Kummer, M. \& Rod, D. L. ${ }_{1983}$ On averaging, reduction, and symmetry in Hamiltonian systems. J. diff Eq. 49, 359-414.

Churchill, R. G., Pecelli, G. \& Rod, D. L. 1975 Isolated unstable periodic orbits. J. diff Eq. 17, 329-348.

Cushman, R. \& Rod, D. L. 1982 Reduction of the semisimple 1:1 resonance. Physica D6, 105-112.

Duistermaat, J. J. 1984 Bifurcations of periodic solutions near equilibrium points of Hamiltonian systems. In Bifurcation theory and applications, Montecatini, 1983 (ed. L. Salvadori) (Lecture notes in mathematics, vol. 1057), pp. 57-105. Berlin: Springer.

Fadell, E. R. \& Rabinowitz, P. H. 1978 Generalized cohomological index theories for Lie group actions with an application to bifurcation questions for Hamiltonian systems. Invent. Math. 45, 139-174.

Fiedler, B. 1986 Habilitationsschrift, Universität Heidelberg.

Gelfand, I. M. \& Lidskii, L. D. 1955 On the structure of stability of linear hamiltonian systems of differential equations with periodic coefficients. Usp. mat. Nauk 10, 3-40. (Amer. math. Soc. Transl. (2) 8, 143-181 (1958).)

Golubitsky, M. \& Stewart, I. N. 1985 Hopf bifurcation in the presence of symmetry. Arch ration. Mech. Analysis 87, 107-165.

Golubitsky, M. \& Stewart, I. N. 1986 Hopf bifurcation with dihedral group symmetry: coupled nonlinear oscillators. In Multiparameter bifurcation theory (ed. M. Golubitsky \& J. Guckenheimer) (Contemporary Math. 56), 131-173. Providence: American Mathematical Society.

Golubitsky, M. \& Stewart, I. N. 1987 Generic bifurcation of Hamiltonian systems with symmetry. Physica D24, 391-405.

Golubitsky, M., Stewart, I. N. \& Schaeffer, D. G. 1988 Singularities and groups in bifurcation theory, vol. 2. New York: Springer. (In the press.)

Guillemin, V. \& Sternberg, S. 1984 Symplectic techniques in physics. Cambridge University Press.

Hale, J. K. 1969 Ordinary differential equations. New York: Wiley-Interscience.

Hartman, P. 1964 Ordinary differential equations. New York: Wiley.

Hénon, M. ${ }_{1983}$ Numerical exploration of Hamiltonian systems. In Chaotic behaviour of deterministic systems (ed. G. Iooss, R. H. G. Helleman \& R. Stora), pp. 55-170. Amsterdam: North-Holland.

Hénon, M. \& Heiles, C. 1964 The applicability of the third integral of motion; some numerical experiments. Astronom. J. 69, 73-79.

Holm, D. D., Marsden, J. E., Ratiu, T. \& Weinstein, A. 1985 Nonlinear stability of fluid and plasma equilibria. Phys. Rep. 123, 1-116.

Krein, M. G. 1950 A generalization of some investigations of A. M. Lyapunov on linear differential equations with periodic coefficients. Dokl. Akad. Nauk SSSR 73, 445-448.

Krein, M. G. 1955 The basic propositions of the theory of $\lambda$-zones of stability of a canonical system of linear differential equations with periodic coefficients. In Topics in differential and integral equations and operator theory (by M. G. Krein). (Operator theory, vol. 7. Basel: Birkhaüser (1983).) (Translated from Izdat. Akad. Nauk SSSR, 413-498 (1955).)

Lamb, H. 1932 Hydrodynamics. 6th edn, Cambridge University Press.

Lancaster, P. \& Tismenetsky, M. 1985 The theory of matrices. New York: Academic Press.

Liapunov, A. M. 1907 Problème générale de la Stabilité du Mouvement. Ann. Fac. Sci. Toulouse 9. (Reprinted Princeton University Press 1947.) (Russian original 1895.)

Losco, L. 1983 Symplectic structures and stability in celestial mechanics. In Symplectic geometry (ed. A. Crumeyrolle \& J. Grifone) (Research notes in mathematics, vol. 80), pp. 122-143. London: Pitman.

MacDonald, I. 1979 Algebraic structure of Lie groups. In Representation theory of Lie groups (ed. M. F. Atiyah) (London Mathematics Society lecture notes, vol. 34), pp. 91-150. Cambridge University Press.

MacKay, R. S. 1986 Stability of equilibria of Hamiltonian systems. In Nonlinear phenomena and chaos (ed. S. Sarkar), pp. 254-270. Bristol: Adam Hilger.

Marsden, J. E. \& Weinstein, A. 1974 Reduction of symplectic manifolds with symmetry. Rep. Math. Phys. 5, 121-130.

Montaldi, J. A., Roberts, R. M. \& Stewart, I. N. I988a Nonlinear normal modes of symmetric Hamiltonian systems. In Proc. Conf. Pattern Formation in Physics, Tübingen, 1986. Berlin: Springer. (In the press.) 
Montaldi, J. A., Roberts, R. M. \& Stewart, I. N. $1988 b$ Existence of nonlinear normal modes of symmetric Hamiltonian systems. Preprint, Mathematics Institute, University of Warwick.

Montaldi, J. A., Roberts, R. M. \& Stewart, I. N. 1988c Stability of nonlinear normal modes of symmetric Hamiltonian systems. Preprint, Mathematics Institute, University of Warwick.

Moser, J. 1958 New aspects in the theory of stability of Hamiltonian systems. Communs Pure appl. Math. 11, 81-114.

Moser, J. 1976 Periodic orbits near equilibrium and a theorem by Alan Weinstein. Communs Pure appl. Math. 29, 727-747.

Palais, R. 1979 The principle of symmetric criticality. Communs math. Phys. 69, 19-30.

Rod, D. L. \& Churchill, R. C. 1985 A guide to the Hénon-Heiles Hamiltonian. In Singularities and dynamical systems (ed. S. Pneumatikos) (Math. Studies 103), pp. 385-395. Amsterdam: North-Holland.

Schmidt, D. S. 1976 Hopf's bifurcation theorem and the center theorem. In The Hopf bifurcation and its applications (ed. J. E. Marsden \& M. McCracken) (Appl. math. Sci. 19), pp. 95-103. New York: Springer.

Springer, T. A. 1981 Linear algebraic groups. Boston: Birkhaüser.

Swift, J. W. 1986 Four coupled oscillators: Hopf bifurcation with the symmetry of a square. Preprint. King's College Research Centre, Cambridge.

Sugiura, M. 1959 Conjugate classes of Cartan subalgebras in real semisimple Lie algebras. J. math. Soc. Japan 11, $374-434$.

Tits, J. I $983_{3}$ Liesche Gruppen und Algebren. Berlin: Springer.

Van Gils, S. A. 1984 Some studies in dynamical system theory. Ph.D. thesis, Vrije Universiteit, Amsterdam.

Varadarajan, V. S. 1974 Lie Groups, Lie Algebras, and their representations. New York: Prentice Hall. (2nd edn New York: Springer (1984).)

Weinstein, A. 1973 Normal modes for nonlinear Hamiltonian systems. Invent. Math. 20, 47-57.

Weinstein, A. 1978 Bifurcations and Hamilton's principle. Math. Z. 159, 235-248.

Whittaker, E. T. \& Watson, G. N. $1927 A$ course of modern analysis, 4th edn. Cambridge University Press.

Williamson, J. 1936 On the algebraic problem concerning the normal forms of linear dynamical systems. Am. $J$. Math. 58, 141-163. 